LA-suB--96-99-Pt. 4

Final Report

DOE Subcontract No. 63545L0014-31

\title{
Measurement of Air Quality Within Storage Domes in Technical Area 54, Area G
}

Prepared by:

Radian Corporation 8501 Mo-Pac Boulevard P.O. Box 201088 Austin, Texas 78720-1088

Technical Collaboration:

Dr. Erik L. Vold CST-14 Solid Radioactive Waste Management MS J595

Los Alamos National Laboratory Los Alamos, New Mexico 87545 

RCN 640-015-01-02 DCN 95-640-015-04

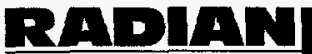

INTERNATIONALLL

(Mailing) P.0. Box 201088

Austin, TX 78720-1088

(Shipping) 8501 N. Mopac Blvd.

Austin, TX 78759

(512) $454-4797$

MEASUREMENT OF AIR QUALITY WITHIN STORAGE DOMES

IN TECHNICAL AREA 54, AREAS G AND L

Final Report

DOE Contract No. 6355L0014-31

\author{
Prepared by: \\ Eric Anderson \\ Radian Corporation \\ 8501 North Mopac Boulevard \\ P. O. Box 201088 \\ Austin, Texas $78720-1088$
}

Technical Collaboration:

Dr. Erik L. Vold

CST-14 Solid Radioactive Waste Management

MS J595

Los Alamos National Laboratory

Los Alamos, New Mexico 87545

March 15, 1994 



\section{DISCLAIMER}

Portions of this document may be illegible in electronic image products. Images are produced from the best available original document. 



\section{TABLE OF CONTENTS}

Page

Abstract $\ldots \ldots \ldots \ldots \ldots \ldots \ldots \ldots \ldots \ldots \ldots \ldots \ldots \ldots, v$

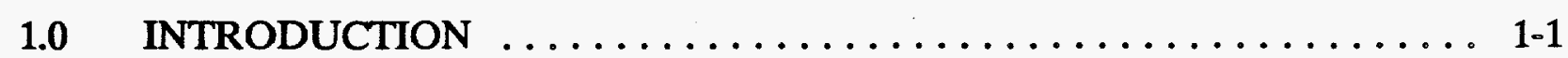

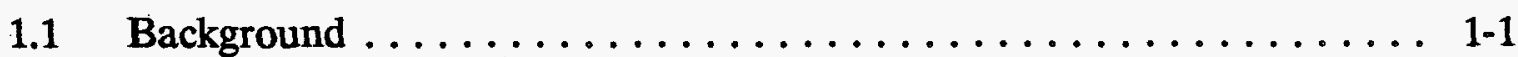

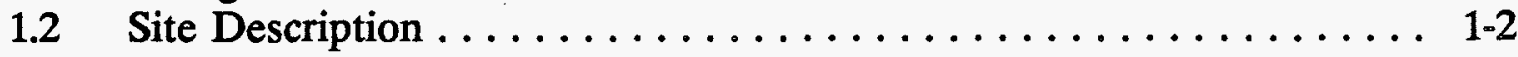

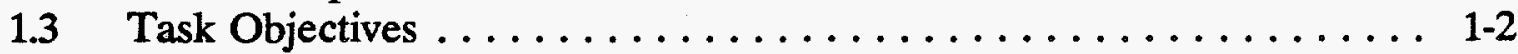

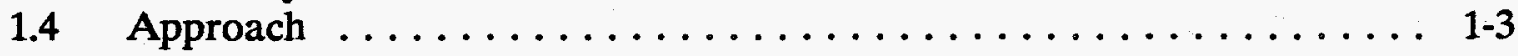

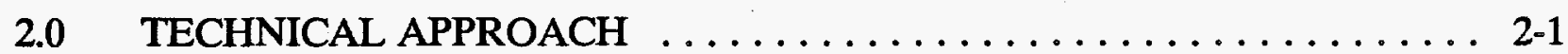

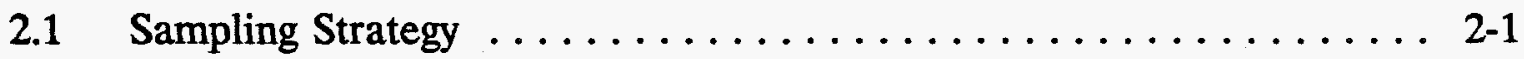

2.2 Tritium Sampling Procedures $\ldots \ldots \ldots \ldots \ldots \ldots \ldots \ldots \ldots \ldots .2-1$

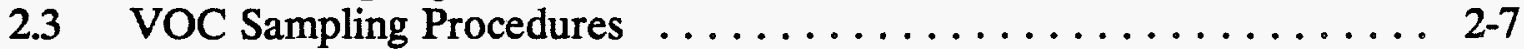

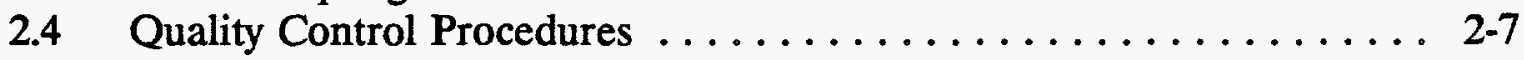

3.0 ANALYTICAL AND QUALITY CONTROL RESULTS $\ldots \ldots \ldots \ldots \ldots$ 3-1

3.1 Tritium Monitoring Results $\ldots \ldots \ldots \ldots \ldots \ldots \ldots \ldots \ldots \ldots$ 3-1

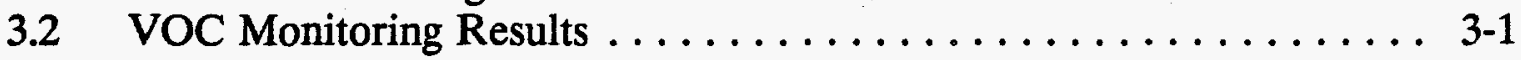

3.3 Meteorological Data $\ldots \ldots \ldots \ldots \ldots \ldots \ldots \ldots \ldots \ldots \ldots . \ldots \ldots . \ldots \ldots$

4.0 DISCUSSION OF RESULTS $\ldots \ldots \ldots \ldots \ldots \ldots \ldots \ldots \ldots \ldots \ldots \ldots \ldots \ldots \ldots \ldots$

4.1 Spatial Variability within Domes $\ldots \ldots \ldots \ldots \ldots \ldots \ldots \ldots \ldots$ 4-1

4.2 Variability of Analyte Concentration Among Domes ......... 4.5

4.3 Effects of Operating Practices on Air Quality ............. 4-6

4.4 Comparison of Measured Values with Applicable Health Standards . 4 4-8

4.5 Estimated Dome Emissions $\ldots \ldots \ldots \ldots \ldots \ldots \ldots \ldots \ldots \ldots$ 4-8

5.0 CONCLUSIONS AND RECOMMENDATIONS $\ldots \ldots \ldots \ldots \ldots \ldots$ 5-1

APPENDIX A: $\quad$ LANL Sampling Master Log $\ldots \ldots \ldots \ldots \ldots \ldots$ A-1 


\section{LIST OF TABLES}

Page

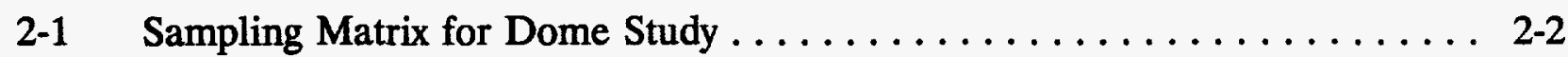

3-1 Summary of Tritium Concentrations - Dome $49 \ldots \ldots \ldots \ldots \ldots \ldots$ 3-2

3-2 Summary of Tritium Results - Dome $48 \ldots \ldots \ldots \ldots \ldots \ldots$. . . . . . .

3-3 Summary of Tritium Results - Dome $153 \ldots \ldots \ldots \ldots \ldots$ 3-4

3-4 Summary of Tritium Sampling Precision $\ldots \ldots \ldots \ldots \ldots \ldots \ldots \ldots \ldots$

3-5 Summary of VOC Results - Dome $48 \ldots \ldots \ldots \ldots \ldots \ldots \ldots$ 3-6

3-6 Summary of Dome 49 voC Results $\ldots \ldots \ldots \ldots \ldots \ldots \ldots \ldots$ 3-9.

3-7 Summary of Dome 153 VOC Results $\ldots \ldots \ldots \ldots \ldots \ldots \ldots \ldots \ldots \ldots \ldots$

3-8 Summary of VOC Sampling Variability (Dome Study Only) $\ldots \ldots \ldots \ldots$ 3-16

3-9 Summary of Area L VOC Results . . . . . . . . . . . . . .

4-1 Summary of Tritium Variability Estimates $\ldots \ldots \ldots \ldots \ldots \ldots \ldots \ldots$ 4-2

4-2 Summary of VOC Spatial Variability Estimates $\ldots \ldots \ldots \ldots \ldots \ldots$ 4-3

4-3 Comparison of Analyte Concentration Based on Dome Condition . . . . . 4-7

4-4 Comparison of Measured Analyte Values with ACGIH TWAs - Domes Closed4-9

4-5 Comparison of Measured Analyte Values with ACGIH TWAs - Domes Open 4-10

4-6 Summary of Annual Dome Emissions for Tritium and Selected VOCs . . . 4-13 


\section{LIST OF FIGURES}

Page

2-1 Location of Sampling Sites in Dome $48 \ldots \ldots \ldots \ldots \ldots \ldots . \ldots . \ldots . \ldots$

2-2 Location of Sampling Sites in Dome $49 \ldots \ldots \ldots \ldots \ldots \ldots \ldots$

2-3 Location of Sampling Sites in Dome $153 \ldots \ldots \ldots \ldots \ldots \ldots$

2-4 Diagram of Tritium Sampling System $\ldots \ldots \ldots \ldots \ldots \ldots \ldots \ldots \ldots$

2-5 Diagram of VOC Sampling System $\ldots \ldots \ldots \ldots \ldots \ldots \ldots \ldots \ldots .2-8$

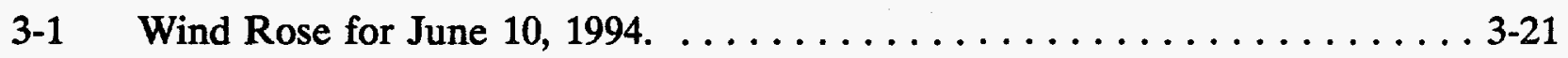

3-2 Wind Rose for June $13,1994 \ldots \ldots \ldots \ldots \ldots \ldots \ldots \ldots \ldots \ldots \ldots \ldots$ 3-22

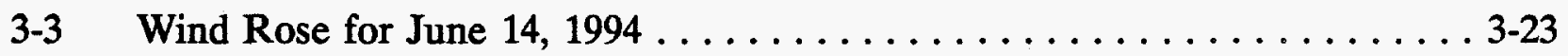

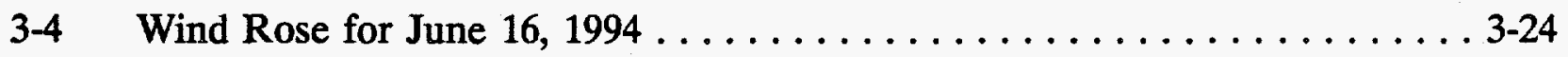

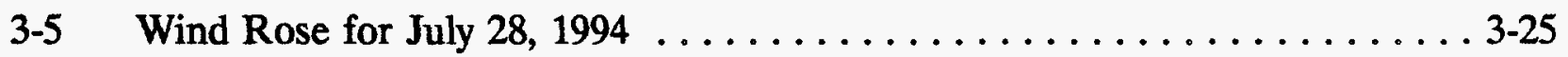

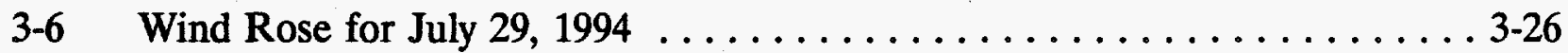

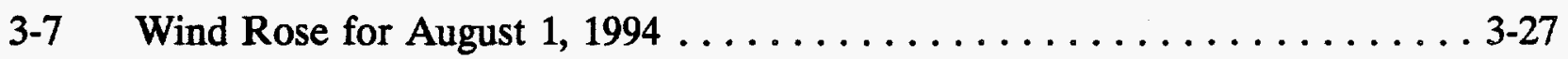

4-1 Comparison of Selected Analytes with ACGIH IWAs or DOE DACs . . . . 4-11 


\title{
Measurement of Air Quality Within Storage Domes in Technical Area 54, Area G
}

\begin{abstract}
The concentrations of volatile organic compounds (VOCs) and tritium inside of storage domes at TA-54 were measured to assess worker exposure and support the Area G site characterization, including the Radioactive Air Emissions Management (RAEM) program. Samples were collected at 2-3 locations within Domes 48,49 , and 153 on up to six days during the summer of 1994. Samples were collected to evaluate three scenarios: 1) normal working activities with the domes open; 2) after domes were closed overnight; and 3) after domes were closed for three days. Eight-hour integrated samples were collected and analyzed in Radian's Austin laboratories.

Tritium activities from 17.1 to $69,900 \mathrm{pCi} / \mathrm{m}^{3}$ were measured. About two dozen individual VOCs were identified in each sample, but most of the concentration levels were very low (e.g.; $<1$ to $10 \mathrm{ppbv)}$. The highest concentrations measured were bromomethane $(56.5$ ppbv), 1,1,1-trichloroethane ( $75.4 \mathrm{ppbv}$ ), propane (958 ppbv), methylene chloride (1,450 ppbv), and toluene (22.8). The measured VOC concentrations were well below the action levels developed by the New Mexico Environment Department and the measured tritium concentrations were well below the DOE's derived air concentration (DAC). The variability in concentration within a dome during a single sampling episode was small. The concentrations were about an order of magnitude (i.e., 10x) higher after the domes had been closed overnight compared with the domes when open. Closing the domes over the weekend did not result in significantly higher concentrations (e.g.; $>20 \%$ ) than when the domes were closed only overnight. The data were used to generate estimated annual dome emission rates of $0.3 \mathrm{Ci} / \mathrm{yr}$ of tritium and less than 100 $\mathrm{lbs} / \mathrm{yr}$ of VOCs.

The measured VOC concentrations were collected during the warmest months of the year and therefore should represent worst-case air impacts. Based on the results of this study, the domes are relatively insignificant emitters of VOCs and tritium. The air quality within the domes does not pose a significant health risk to workers nor does it account for a significant portion of the allowable annual exposure.
\end{abstract}





\subsection{INTRODUCTION}

Los Alamos National Laboratory (LANL) has operated a disposal facility for low-level radioactive solids in Area $G$ of Technical Area 54 (TA-54) since 1957. A smaller adjacent disposal area, designated Area L, is used for the storage of lowlevel mixed waste. Various types of wastes have historically been disposed of in TA-54, including tritium waste, transuranic element (TRU) waste, volatile organic compounds (VOCs) such as solvents, and mixed waste. Currently, however, only low-level radioactive waste is disposed of at TA-54. The waste disposal practices in Area $\mathbf{G}$ are designed to contain the waste and prevent or minimize migration of contaminants via all pathways, including the air pathway.

Waste disposal practices include burial in shaft and pits as well as temporary storage in above ground storage domes. To evaluate the level of waste containment, environmental monitoring has been routinely performed by LANL to measure possible migration of contaminants. The air pathway is addressed by a fenceline network of fixed sites used to monitor the ambient air for tritium, TRU, and alpha, beta, and isotopic gamma radiation. Additional monitoring is performed by LANL to examine concentration levels of various contaminants in the surface soils, vegetation, pore gas, and runoff.

The environmental monitoring to date has indicated that no significant air emissions of radioactive species at TA-54 has occurred during the ambient air monitoring (AAM) program. There could, however, be relatively low-level emissions or localized emission "hot spots" that need to be characterized. The gas-phase contaminants that are the most mobile are tritium and VOCs; therefore, these species could be emitted from the waste stored in the domes and could affect air quality if released into the atmosphere.

\subsection{Background}

Some waste forms, including TRU and mixed waste, are temporarily stored in large "domes" prior to off-site disposal. In addition, tritiated waste and VOC containing waste is also temporarily stored in Area $L$. This study evaluated the maximum concentrations of VOCs and tritium inside the Area $\mathrm{G}$ domes (including Dome 49, operated by Area $L$ personnel), determined the "typical" concentrations that workers within the domes may be exposed to during a normal work-day, and assessed the emissions from the stored waste within Area $L$.

This study is being performed for several reasons:

- $\quad$ Emissions data are needed to support the Radioactive Air Emissions Management (RAEM) program's characterization of radioactive air emissions from waste sites to assure compliance with the National Emission Standards for Hazardous Air Pollutants (NESHAP) for radionuclides.

- $\quad$ Although not a regulatory requirement, there is a need to characterize non-radioactive air 
emissions to determine if the Laboratory's waste operations are releasing toxic amounts of pollutants.

- $\quad$ The Department of Energy (DOE) requires that all facilities follow Best Management Practices (BMP) with regards to all of their activities. Quantifying releases of pollutants, even though not required by regulators, is a BMP.

\subsection{Site Description}

TA-54 occupies about 63 acres on the southern half of Mesita del Buey on the Pajarito Plateau. Mesita del Buey is a narrow mesa about 2 miles long that extends to the southeast. The elevation of the mesa is roughly $6900 \mathrm{ft}$. on the west and $6600 \mathrm{ft}$. at the eastern end. The mesa is bounded by Pajarito Canyon to the south and Canada del Buey to the north. The edges of the mesa have been cut to near vertical and vary in height from 50 to 100 $\mathrm{ft}$. Areas $\mathrm{G}$ and $\mathrm{L}$ are located on Mesita del Buey approximately 4.5 miles southeast of the town of Los Alamos and 1 mile northwest of the subdivision of White Rock. Area $\mathrm{G}$ occupies over 60 acres at the southern end of TA-54 while Area L occupies several acres immediately west of Area G within TA-54.

The tritium waste disposed of in shafts at Area G, is solid waste contaminated with tritium in excess of 20 $\mathrm{mCi} / \mathrm{m}^{3}$ (waste with levels below 20 $\mathrm{mCi} / \mathrm{m}^{3}$ is disposed of as routine low-level waste in pits). Waste contaminated with tritium between 20 and $100 \mathrm{mCi} / \mathrm{m}^{3}$ is packaged in 55-gallon or 30-gallon, asphalt-lined metal drums. The lids of the drums are sealed with asphalt and the waste is disposed of in unlined shafts. Tritium waste with levels exceeding 100 $\mathrm{mCi} / \mathrm{m}^{3}$ must be completely encapsulated in asphalt. This typically involves putting a 30-gallon drum inside a 55-gallon drum and filling the annular space between the two drums with asphalt. These drums are disposed of in asphalt-lined shafts.

TRU wastes, contained in 55-gallon drums, fiberglass reinforced plywood (FRP) boxes, or in standard waste boxes are stored above-ground in temporary storage domes prior to final disposal. Area $L$ is a paved area used for temporary above-ground storage of drums of mixed wastes and gas cylinders.

There are currently three domes inside of Area $G$ that are used for temporary storage. Dome 48 is located in the northeastern corner of Area $G$ and is used for storage of certified TRU waste. This dome is approximately 285 feet long and 50 feet wide. Dome 153 is located in the middle of Area $G$ and is used for storage of fiberglass reinforced plywood (FRP) boxes. This dome is approximately 325 feet long and 60 feet wide. Dome 49 is operated by Area $L$ and is used for storage of mixed wastes. This dome is approximately the same size as Dome 153 .

\subsection{Task Objectives}

The main objectives of this program were to measure tritium and VOC concentrations within the three storage domes at the breathing zone (i.e., 5 feet above floor level). This was done under 
both open and closed conditions, which should provide both normal working levels and "worst" case concentrations. Two closed conditions were evaluated, overnight closure and over-weekend closure. This was done to see if levels continued to increase over time when the domes were closed. In addition, a single VOC monitoring run was performed in the area where mixed wastes are stored inside of Area L. This area may eventually be covered by a dome, and the measurement data represent existing air quality in the area surrounding these wastes.

The specific objectives of this program were to:

- Develop emission estimates from the domes and analyte concentrations in the storage domes (worst-case and under "normal" operation) so air handling and/or emission control systems can be designed;

- $\quad$ Assess the potential for worker exposure within the domes; and

- $\quad$ Assess air quality of the drum storage area prior to construction of a storage dome.

\subsection{Approach}

Three domes in Area $\mathrm{G}$ were characterized to determine concentrations of VOCs and tritiated water and VOCs inside the structures. The VOC samples were collected using SUMMA ${ }^{\oplus}$ polished stainless steel canisters while the tritiated water samples were collected on silica gel sorbent cartridges. The testing was conducted under three different scenario's. Scenario 1: One set of samples were collected after the domes had been closed overnight. This provides an estimate of the concentrations of VOCs and tritiated water that might be expected inside the structures each morning. Scenario 2: Another set of samples were collected after the domes had been closed over a weekend. This provides an estimate of the maximum concentrations that occur inside the domes. Scenario 3: The third set of samples were collected during "normal" working conditions, i.e., domes open and naturally ventilated.

Samples were collected over approximately 8-hour periods. For Domes 48 and 153 , samples were collected during June and again in July. This was done to evaluate the temporal variability of the VOC and tritiated water concentrations.

The data sets collected during this study provide useful information, but are in no way exhaustive. All samples were collected at the breathing zone, so information on the vertical stratification (if any exists) can not be assessed.

Additionally, since no measurements of air movement within the domes were performed, emission estimates are based on assumptions of assumed ventilation rates within the domes. 

TA-54 Dome Study

\subsection{TECHNICAL APPROACH}

This section describes the sampling and analytical procedures that were used to collect and quantitate the VOC and tritium samples from the domes in Area $\mathbf{G}$ and the storage area in Area L.

\subsection{Sampling Strategy}

VOC and tritium samples were collected inside three domes within Area G, the certified TRU waste dome (Dome 48), FRP storage dome (Dome 153), and the low-level mixed waste dome (Dome 49), operated by Area L personnel. In addition, VOC samples were collected inside Area $L$, along an asphalt pad where various mixed waste is stored. For domes 48 and 153, three sets of samples were collected during June and again in July. A single set of three samples were collected from Dome 49 during July. A single set of three samples were collected from the mixed waste storage pad in Area $L$. The domes located inside Area $G$ were sampled under two conditions. The first represented a "worst-case" condition when the domes were closed (sampling occurred after domes were closed overnight and over a weekend). The second occurred during normal facility operation (i.e., doors open and naturally ventilated). This provides both maximum expected analyte concentrations as well as analyte concentrations during working hours. Table 2-1 presents the test matrix for the sampling program.

Domes 153 and 49 are approximately the same size, 325 feet long and 60 feet wide. Dome 48 is smaller, approximately 285 feet long and 50 feet wide. Because of the length of the structures and the fact that there is no active mixing of the air, the potential for stratification of the constituents is likely. Therefore, three sampling locations were monitored within Domes 153 and 49 and two locations were monitored within Dome 48. Figures $2-1$ through $2-3$ show the locations of the monitoring locations in each of the three domes. Integrated samples were collected over an 8-hour period during each monitoring episode.

VOC samples from the fugitive emissions of waste stored in Area $L$ were collected during June. Waste is typically stored in this area in 30 to 55 gallon drums, depending on the waste type and characteristics. Samples were collected by placing VOC canisters between the rows of waste drums (two samples) and at the end of the storage pad (downwind of the drums). Samples were collected over an 8-hour period on a day having light winds.

\subsection{Tritium Sampling Procedures}

Tritium samples were collected as tritiated water vapor using silica gel sorbent cartridges. A pump was used to pull the air inside the domes through the sorbent cartridges at a rate in the range of 3 to 4 liters-per-minute. A dry gas meter was used to measure the total volume of gas sampled.

Two silica gel cartridges, containing approximately 40 grams of silica gel each, (each 1 inch O.D. by 6 inches long) arranged in series were used to collect the tritiated water samples. Figure 2-4 shows a diagram of the 


\section{Table 2-1. Sampling Matrix for Dome Study}

\begin{tabular}{|c|c|c|c|c|}
\hline Bome & $\begin{array}{l}\text { Sampling: } \\
\text { linterval'? }\end{array}$ & Nocations & Analytes Monitored & Conditions \\
\hline Dome 153 & $2^{1}$ & 3 & $\begin{array}{c}\text { Tritium and VOCs (8-hour } \\
\text { integrated) }\end{array}$ & $\begin{array}{l}\text { Dome open and monitored during } \\
\text { normal working activities }\end{array}$ \\
\hline Dome 153 & $2^{1}$ & 3 & $\begin{array}{c}\text { Tritium and VOCs (8-hour } \\
\text { integrated) }\end{array}$ & $\begin{array}{l}\text { Dome closed overnight, monitoring } \\
\text { occurred while dome still closed }\end{array}$ \\
\hline Dome 153 & $2^{1}$ & 3 & $\begin{array}{c}\text { Tritium and VOCs (8-hour } \\
\text { integrated) }\end{array}$ & $\begin{array}{l}\text { Dome closed from Friday through } \\
\text { Monday, monitoring occurred while } \\
\text { dome still closed }\end{array}$ \\
\hline Dome 49 & 1 & 3 & $\begin{array}{c}\text { Tritium and VOCs (8-hour } \\
\text { integrated) }\end{array}$ & $\begin{array}{l}\text { Dome open and monitored during } \\
\text { normal working activities }\end{array}$ \\
\hline Dome 49 & 1 & 3 & $\begin{array}{c}\text { Tritium and VOCs (8-hour } \\
\text { integrated) }\end{array}$ & $\begin{array}{l}\text { Dome closed overnight, monitoring } \\
\text { occurred while dome still closed }\end{array}$ \\
\hline Dome 49 & 1 & 3 & $\begin{array}{c}\text { Tritium and VOCs (8-hour } \\
\text { integrated) }\end{array}$ & $\begin{array}{l}\text { Dome closed from Friday through } \\
\text { Monday, monitoring occurred while } \\
\text { dome still closed }\end{array}$ \\
\hline Dome 48 & $2^{1}$ & 2 & $\begin{array}{c}\text { Tritium and VOCs (8-hour } \\
\text { integrated) }\end{array}$ & $\begin{array}{l}\text { Dome open and monitored during } \\
\text { normal working activities }\end{array}$ \\
\hline Dome 48 & $2^{\prime}$ & 2 & $\begin{array}{c}\text { Tritium and VOCs (8-hour } \\
\text { integrated) }\end{array}$ & $\begin{array}{l}\text { Dome closed overnight, monitoring } \\
\text { occurred while dome still closed }\end{array}$ \\
\hline Dome 48 & $2^{1}$ & 2 & $\begin{array}{c}\text { Tritium and VOCs (8-hour } \\
\text { integrated) }\end{array}$ & $\begin{array}{l}\text { Dome closed from Friday through } \\
\text { Monday, monitoring occurred while } \\
\text { dome still closed }\end{array}$ \\
\hline Area $L$ & 1 & 3 & VOCs (8-hour integrated) & Ambient samples, open air \\
\hline
\end{tabular}

1 A Sampling Interval consists of three sampling runs performed under three different conditions. Sampling interval 1 was during June 1994 and sampling interval 2 was during July and August 1994.

Dome 153 used for storage of FRP boxes.

Dome 49 used for storage of low-level mixed waste.

Dome 48 used for storage of certified TRU waste. 


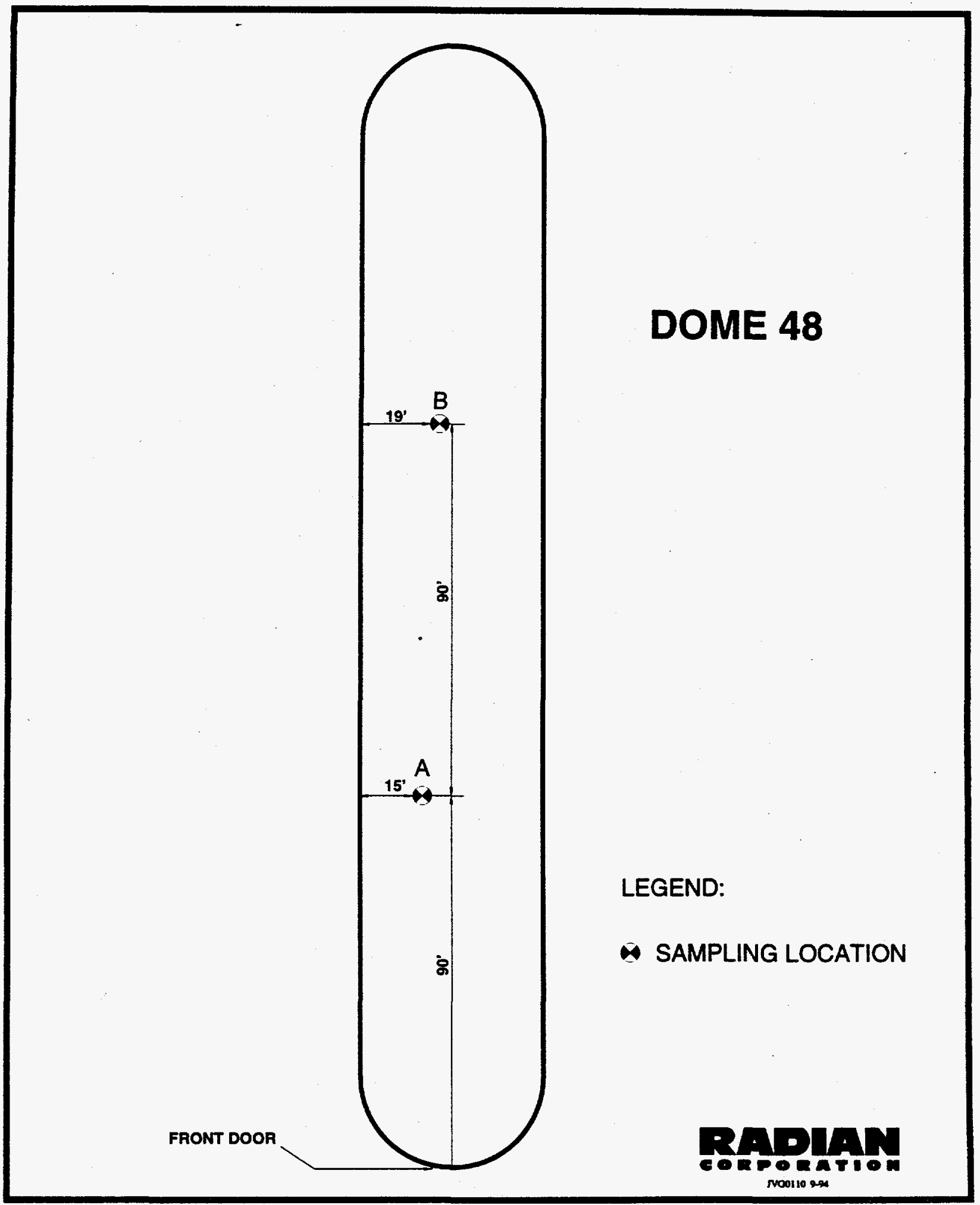

Figure 2-1. Location of Sampling Sites in Dome 48. 


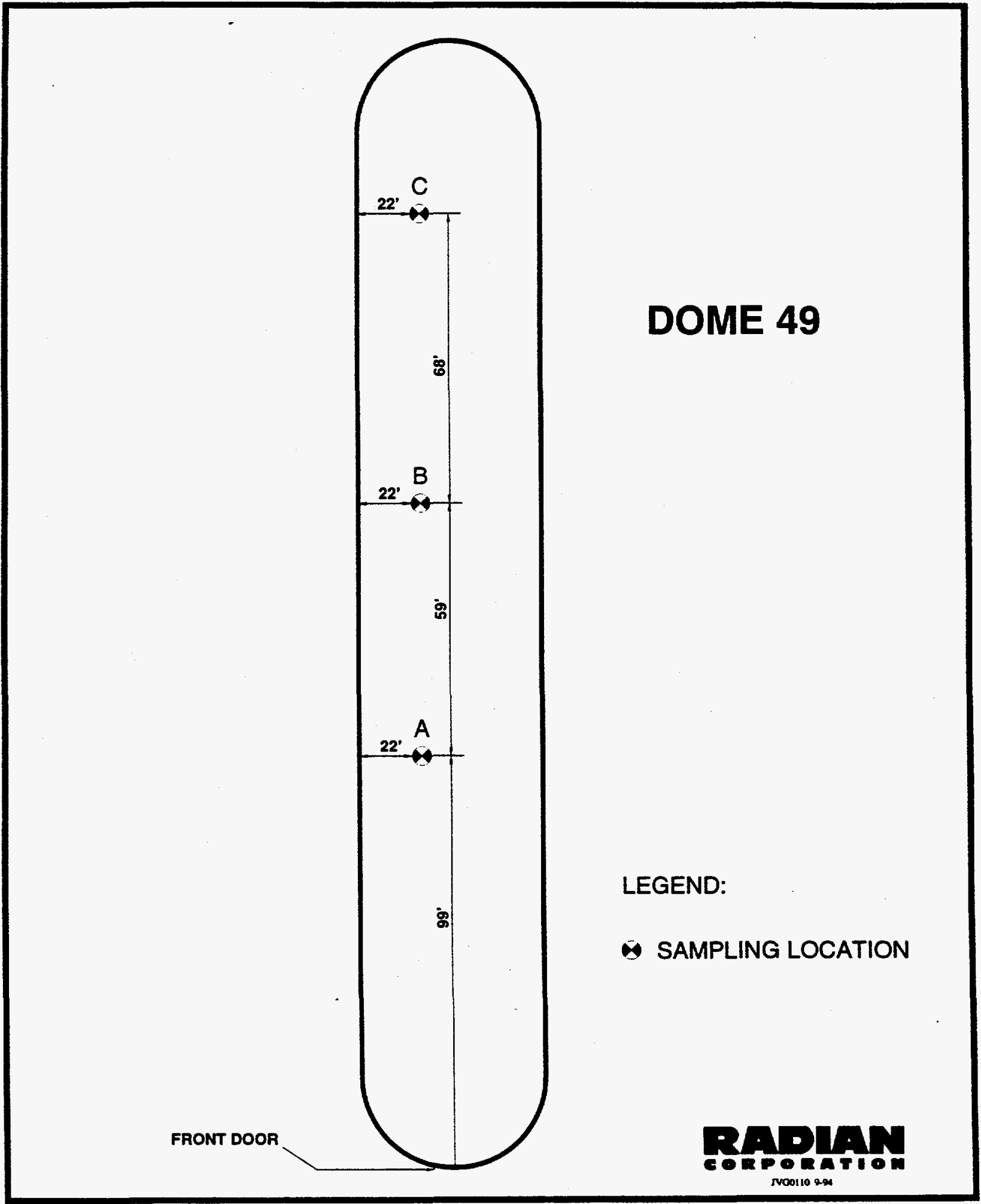

Figure 2-2. Location of Sampling Sites in Dome 49. 


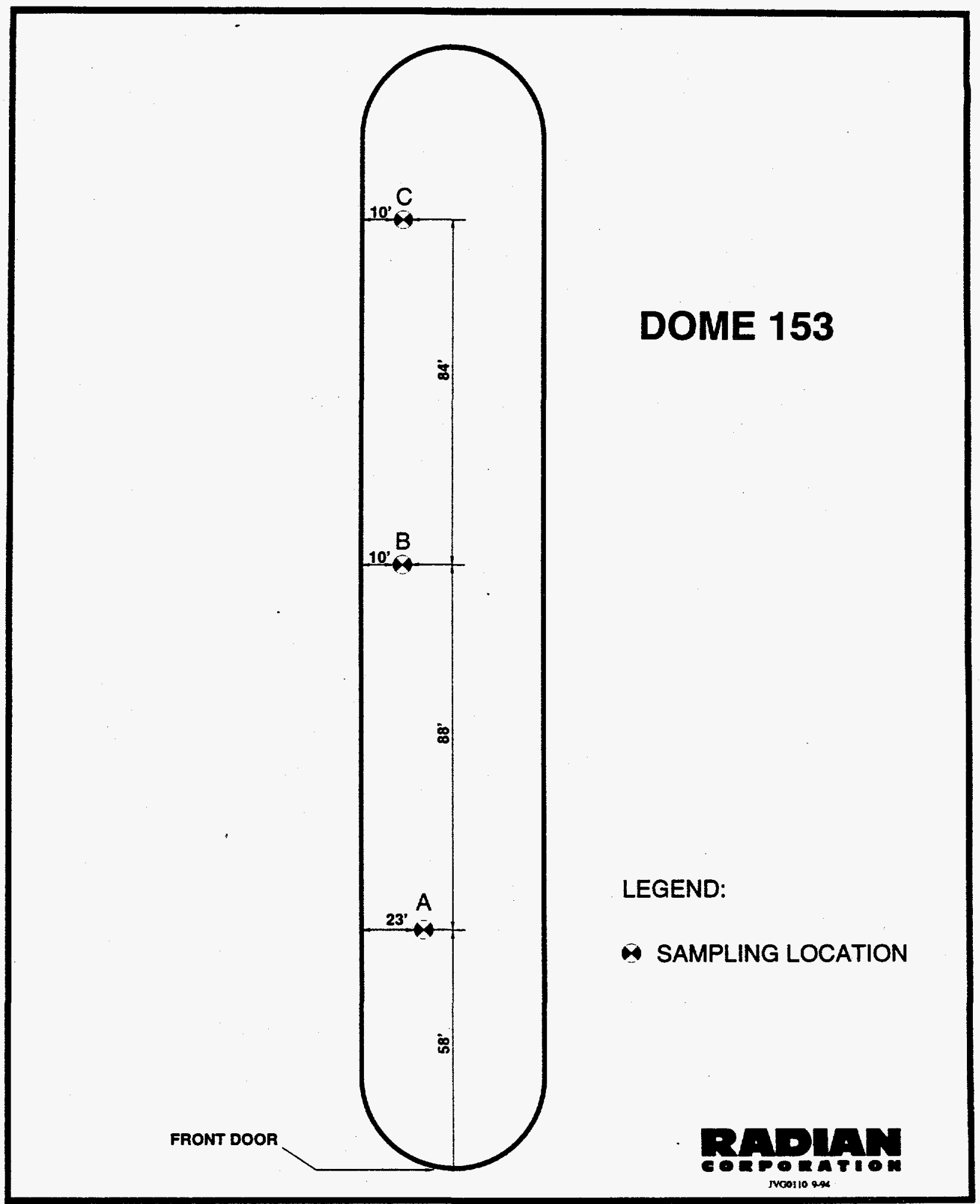

Figure 2-3. Location of Sampling Sites in Dome 153. 


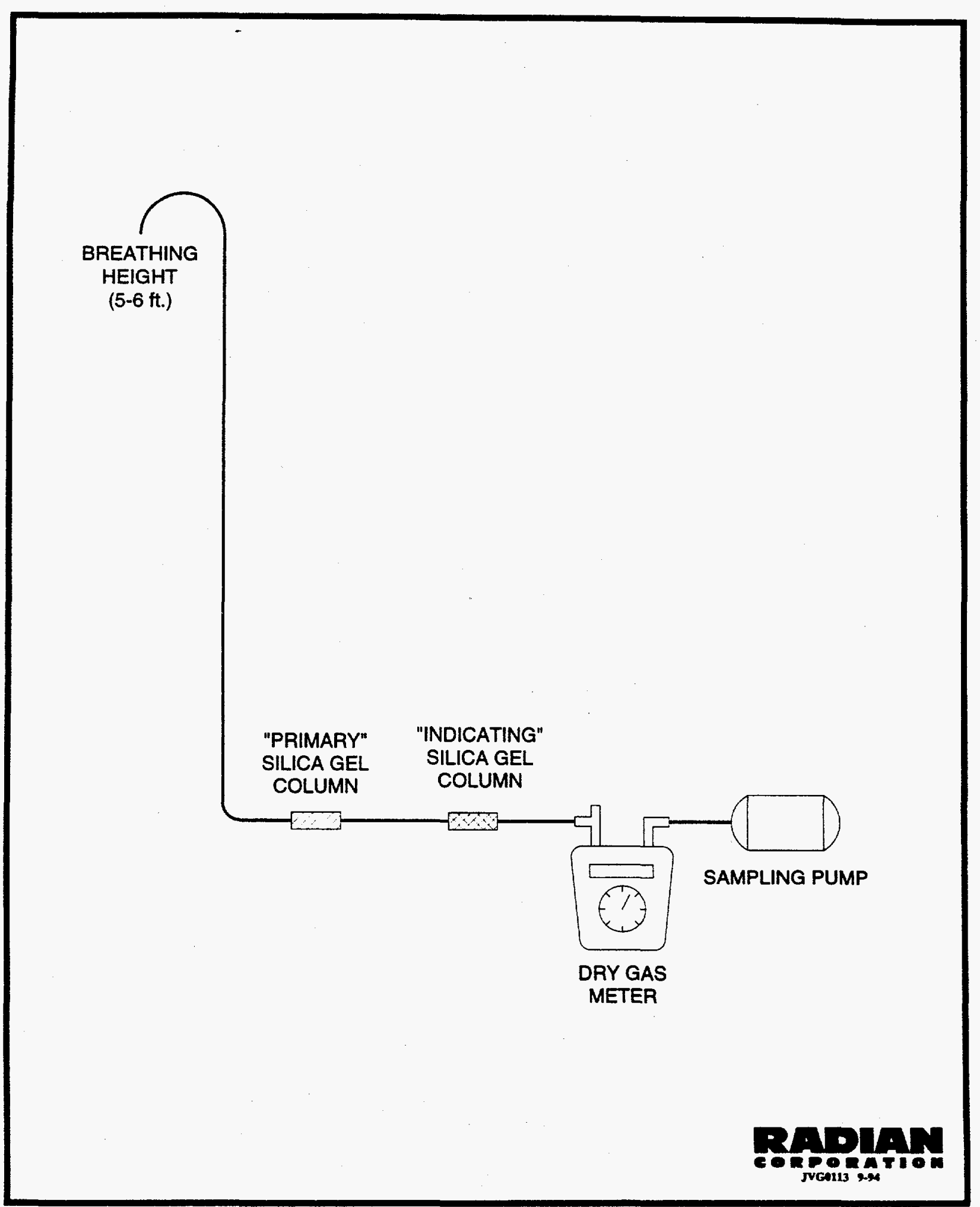

Figure 2-4. Diagram of Tritium Sampling System. 
TA-54 Dome Study

sampling system. The first cartridge consisted of 35-60 mesh silica gel for primary tritiated water adsorption followed by a cartridge containing 6-16 mesh indicating silica gel which turned from blue to pink in the presence of water vapor. Each cartridge was capable of retaining approximately 12 grams of water vapor. The second cartridge was used to determine when the primary sorbent cartridge was saturated, and consequently when sufficient water vapor had been collected for an acceptable analytical detection limit. The runs lasted approximately 8 hours, however, the exact duration of sampling depended upon site logistics and breakthrough characteristics of the sample.

\subsection{VOC Sampling Procedures}

Air samples for the determination of VOCs were collected using evacuated 15-L SUMMA ${ }^{\star}$ passivated stainless steel canisters. A diagram of the sampling system is presented in Figure 2-5.

Each sampler was equipped with a $1 / 4$-inch o.d. stainless steel sampling cane extending approximately 5 feet (breathing zone height) above the ground surface, 7micrometer and 2-micrometer particulate filters, a vacuum gauge and a Milliflow differential flow controller to collect the time-integrated samples. Milliflow regulators were individually adjusted for each canister.

The canisters were prepared for sampling at Radian's VOC Laboratory. Prior to sampling each canister was cleaned and blanked. Each canister must have had a residual VOC concentration below $10 \mathrm{ppbv-C}$ total hydrocarbon.
Following cleaning and blanking, the canisters were evacuated, their vacuum measured, and they were shipped to the field.

During sampling, a calibrated lowflow rotameter was used to set the canister flowrate. Once the samples were started, the flowrates were checked several times during each 8-hour sampling period to ensure the flowrates are correct. The flows were adjusted so that the final canister vacuum was approximately 9 inches Hg. System.

\subsection{Quality Control Procedures}

A number of quality control procedures were employed during the program to help assess the quality of the data being generated. These included both field and laboratory QC procedures. Specific field procedures included collection of collocated samples for VOCs and tritium and preparation of spiked (with "old" aquifer mineral water) silica gel tubes. Laboratory QC procedures were extensive and are detailed in the project specific QAPP titled "Measurement of Air Quality Within Storage Domes in Technical Area 54, Area G" submitted May 25, 1994. 


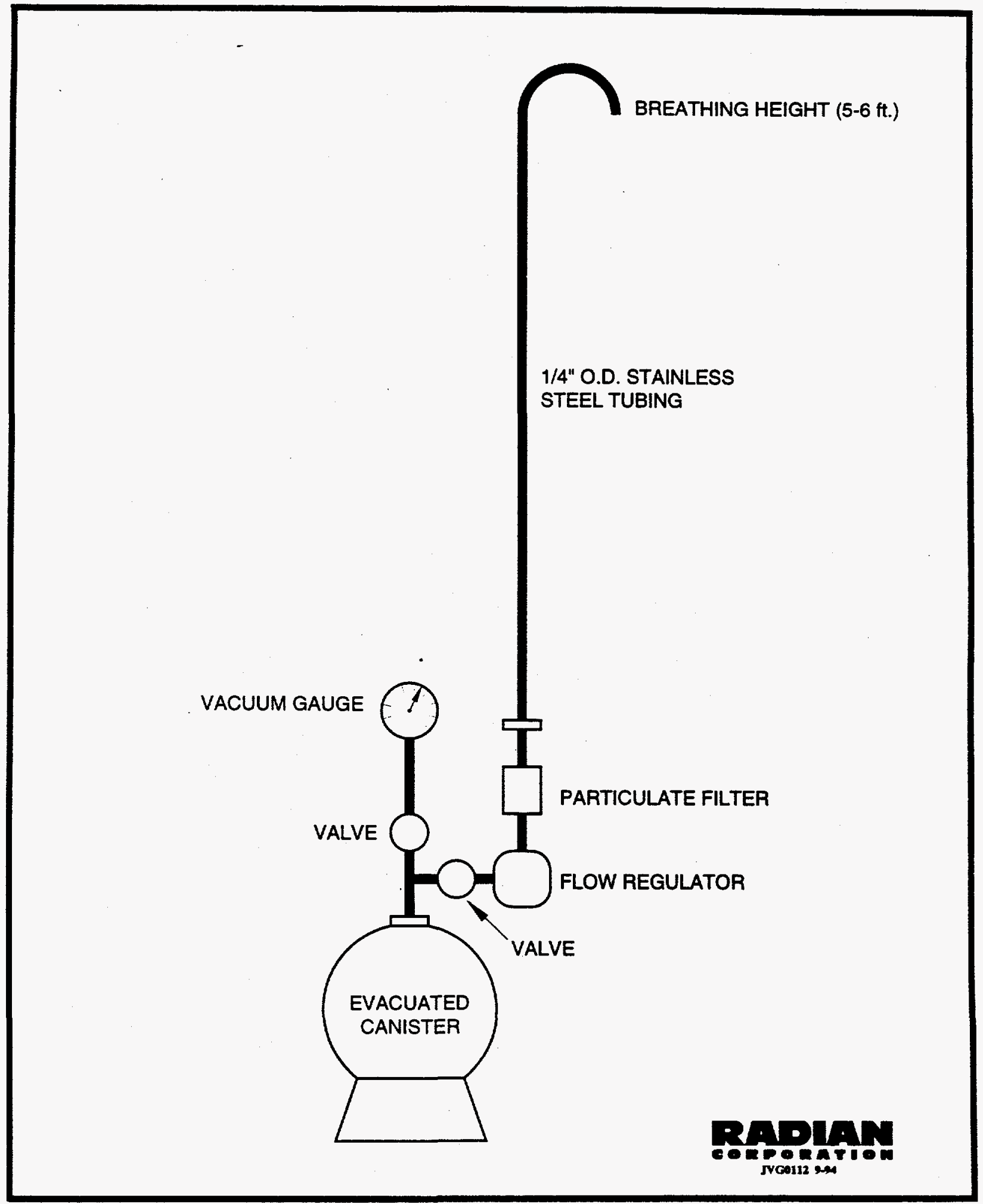

Figure 2-5. Diagram of VOC Sampling System. 


\subsection{ANALYTICAL AND QUALITY CONTROL RESULTS}

This section presents a summary of monitoring and quality control results from the monitoring program. A discussion of the monitoring results is presented in Section 4.0.

\subsection{Tritium Monitoring Results}

Tritium samples were collected inside the storage domes over an approximate 8-hour period under three conditions. The first condition simulated normal operating conditions, i.e., dome door open. The second sample was collected after the domes had been closed overnight and the domes remained closed during the sampling. The last sample was collected after the domes had remained closed over the weekend. This should represent a "worst" case for dome concentrations. Three sampling locations were monitored inside Domes 49 and 153 while two locations were monitored inside Dome 48. The monitoring locations were shown in Figures 2-1 through 2-3 for Domes 48, 49, and 153. Domes 48 and 153 were sampled in both June and July while Dome 49 was sampled in July only. The tritium data are presented in Tables 3-1 through 3-3 for domes 48, 49, and 153 , respectively. Duplicate sampling runs were performed each sampling period. The tritium data are presented in Tables 3-1 through 3-3 for domes 48, 49, and 153 , respectively. The results from the tritium duplicate sampling are shown in Table 3-4.

\subsection{VOC Monitoring Results}

VOC monitoring runs were conducted along with each tritium run in the three storage domes located in Area G. The analytical results for Domes 48, 49, and 153 are shown in Tables 3-5 through 3-7. The results from the duplicate VOC samples (collected during each sampling period) are shown in Table 3-8. The monitoring results from the storage pad inside Area $L$ are presented in Table 3-9.

\subsection{Meteorological Data}

Wind direction and wind speed during the dome sampling have a minimal impact on the generated data (with the exception of Area $L$ sampling). Wind data are presented in the event that LANL would want to try and evaluate the VOC and tritium data with wind direction. Winds blowing directly into an open dome would increase air exchange inside the domes, however, the door would have to be directly downwind in a very narrow vector to have a significant impact. High wind speeds across (i.e. perpendicular to) the door opening could increase air exchange via a venturi effect. Meteorological data, consisting of wind rose plots for each 8-hour sampling period are shown in Figures 3-1 through 3-7 for June 10, June 13, June 14, June 16, July 28, July 29, and August 01, respectively. 


\section{Table 3-1}

Summary of Tritium Concentrations - Dome 49

\begin{tabular}{||c|c|c|c|c||}
\hline $\begin{array}{c}\text { Sample } \\
\text { Date }\end{array}$ & Point & $\begin{array}{c}\text { Grid } \\
\text { Pale Volume } \\
\left(\mathrm{ft}^{3}\right)\end{array}$ & $\begin{array}{c}\text { Total Sample } \\
\text { Activity (pCi) }\end{array}$ & $\begin{array}{c}\text { Ambient Concentration } \\
\left(\mathrm{pCi} / \mathrm{m}^{3}\right)\end{array}$ \\
\hline $07 / 28 / 94$ & $49-\mathrm{A}$ & 45.2 & 216 & 168 \\
\hline $07 / 28 / 94$ & $49-\mathrm{B}$ & 47.3 & 144 & 108 \\
\hline $07 / 28 / 94$ & $49-\mathrm{C}$ & 51.9 & 198 & 135 \\
\hline $07 / 29 / 94$ & $49-\mathrm{A}$ & 41.2 & 401 & 343 \\
\hline $07 / 29 / 94$ & $49-\mathrm{B}$ & 46.7 & 1810 & 1,372 \\
\hline $07 / 29 / 94$ & $49-\mathrm{C}$ & 49.4 & 431 & 308 \\
\hline $08 / 01 / 94$ & $49-\mathrm{A}$ & 34.2 & 482 & 497 \\
\hline $08 / 01 / 94$ & $49-\mathrm{B}$ & 37.5 & 611 & 575 \\
\hline $08 / 01 / 94$ & $49-\mathrm{C}$ & 44.6 & 645 & 511 \\
\hline
\end{tabular}

NOTE: $\quad 7 / 28 / 93$ samples collected with dome open.

$7 / 29 / 94$ samples collected after dome had been closed for 16 hours.

$8 / 01 / 94$ samples collected after dome had been closed for 64 hours. 
Table 3-2

Summary of Tritium Results - Dome 48

\begin{tabular}{|c|c|c|c|c|}
\hline Sample & $\begin{array}{l}\text { Grid } \\
\text { Point }\end{array}$ & $\begin{array}{l}\text { Sample Volume } \\
\text { (ft3) }\end{array}$ & $\begin{array}{l}\text { Total Sample } \\
\text { Activity (pCi) }\end{array}$ & $\begin{array}{l}\text { Ambient Concentration } \\
\text { (pCi/m3) }\end{array}$ \\
\hline \multicolumn{5}{|c|}{ June Results } \\
\hline $06 / 10 / 94$ & 48-A & 55.5 & 15,400 & 9,780 \\
\hline $06 / 10 / 94$ & 48-B & 58.7 & 21,700 & 13,100 \\
\hline $06 / 13 / 94$ & 48-A & 63.4 & 18,700 & 10,400 \\
\hline $06 / 13 / 94$ & 48-B & 61.9 & 23,700 & 13,500 \\
\hline $06 / 14 / 94$ & 48-A & 143.8 & 69.8 & 17.1 \\
\hline $06 / 14 / 94$ & 48-B & 143.2 & 11,100 & 2,730 \\
\hline \multicolumn{5}{|c|}{ July Results } \\
\hline $07 / 28 / 94$ & 48-A & 43.9 & 1,210 & 971 \\
\hline $07 / 28 / 94$ & 48-B & 26.5 & 2,380 & 3,170 \\
\hline $07 / 29 / 94$ & 48-A & 40.5 & 8,740 & 7,620 \\
\hline $07 / 29 / 94$ & 48-B & 32.2 & 9,680 & 10,600 \\
\hline $08 / 01 / 94$ & 48-A & 39.7 & 12,900 & 11,500 \\
\hline 08/01/94 & 48-B & 34.7 & 15,700 & 15,900 \\
\hline
\end{tabular}

NOTE: $\quad 6 / 10 / 94$ samples collected after dome had been closed for 16 hours. 6/13/94 samples collected after dome had been closed for 64 hours. 6/14/94 samples collected with dome open.

7/28/94 samples collected with dome open.

7/29/94 samples collected after dome had been closed for 16 hours. 8/01/94 samples collected after dome had been closed for 64 hours. 
Table 3-3

Summary of Tritium Results - Dome 153

\begin{tabular}{|c|c|c|c|c|}
\hline Sample. & $\begin{array}{l}\text { Grid } \\
\text { Point }\end{array}$ & $\begin{array}{l}\text { Sample } \\
\text { Volume (ft3) }\end{array}$ & $\begin{array}{l}\text { Total Sample } \\
\text { Activity (pCi) }\end{array}$ & $\begin{array}{l}\text { Ambient Concentration } \\
\text { (pCi/m3) }\end{array}$ \\
\hline \multicolumn{5}{|c|}{ June Results } \\
\hline $06 / 10 / 94$ & $153-A$ & 49.4 & 83,900 & 60,000 \\
\hline $06 / 10 / 94$ & 153-B & 56.7 & 94,000 & 58,600 \\
\hline $06 / 10 / 94$ & $153-C$ & 51.9 & 102,000 & 69,100 \\
\hline $06 / 13 / 94$ & 153-A & 60.7 & 96,200 & 55,900 \\
\hline $06 / 13 / 94$ & 153-B & 60.5 & 77,400 & 45,200 \\
\hline $06 / 13 / 94$ & $153-C$ & 60.1 & 100,000 & 58,800 \\
\hline $06 / 14 / 94$ & 153-A & 139.1 & 10,900 & 2,780 \\
\hline $06 / 14 / 94$ & 153-B & 140.6 & 12,200 & 3,060 \\
\hline $06 / 14 / 94$ & 153-C & 130.4 & 27,100 & 7,340 \\
\hline \multicolumn{5}{|c|}{ July Results } \\
\hline $07 / 28 / 94$ & $153-A$ & 42.2 & 5,080 & 4,250 \\
\hline $07 / 28 / 94$ & 153-B & 43.4 & 5,440 & 4,430 \\
\hline $07 / 28 / 94$ & 153-C & 42.4 & 7,120 & 5,930 \\
\hline $07 / 29 / 94$ & 153-A & 24.9 & 38,200 & 54,200 \\
\hline $07 / 29 / 94$ & 153-B & 34.9 & 40,300 & 40,700 \\
\hline $07 / 29 / 94$ & $153-C$ & 33.1 & 53,200 & 56,900 \\
\hline $08 / 01 / 94$ & $153-A$ & 35.5 & 64,900 & 64,600 \\
\hline $8 / 01 / 94$ & 153-B & 47.5 & 79,900 & 59,500 \\
\hline $08 / 01 / 94$ & $153-C$ & 33.4 & 66,100 & 69,900 \\
\hline
\end{tabular}

NOTE: $\quad 6 / 10 / 94$ samples collected after dome had been closed for 16 hours. 5/13/94 samples collected after dome had been closed for 64 hours. $6 / 14 / 94$ samples collected with dome open.

7/28/94 samples collected with dome open

$7 / 29 / 94$ samples collected after dome had been closed for 16 hours. 8/01/94 samples collected after dome had been closed for 64 hours. 


\section{Table 3-4 \\ Summary of Tritium Sampling Precision}

\begin{tabular}{|c|c|c|c||}
\hline Sample Date & Sample $A$ & Sample B & Percent Difference \\
\hline $06 / 10 / 94$ & 13,073 & 13,524 & 3.39 \\
\hline $06 / 13 / 94$ & 13,521 & 11,810 & 13.51 \\
\hline $07 / 28 / 94$ & 3,172 & 2,886 & 9.44 \\
\hline $07 / 29 / 94$ & 10,618 & 10,481 & 1.29 \\
\hline $08 / 01 / 94$ & 15,945 & 15,066 & 5.67 \\
\hline
\end{tabular}

Note: Samples A and B are duplicate samples collected at the same location. 
Table 3-5

Summary of VOC Results - Dome 48

\begin{tabular}{|c|c|c|c|c|c|c|c|c|c|c|c|c|}
\hline \multirow[b]{2}{*}{ Compound } & \multicolumn{2}{|c|}{$6 / 10 / 94$} & \multicolumn{2}{|c|}{$6 / 13 / 94$} & \multicolumn{2}{|c|}{$6 / 14 / 94$} & \multicolumn{2}{|c|}{$7 / 28 / 94$} & \multicolumn{2}{|c|}{$7 / 29 / 94$} & \multicolumn{2}{|c|}{$8 / 01 / 94$} \\
\hline & A & B & A & B & $\mathrm{A}$ & B & A & B & A & B & A & B \\
\hline Ethylene & 3.8 & 4.1 & 2.2 & 2.7 & 0.4 & 0.4 & 0.5 & 0.7 & 2.4 & 2.8 & 2.3 & 2.8 \\
\hline Acetylene & 1.6 & 1.3 & 1.1 & 0.9 & ND & $\mathrm{ND}$ & ND & ND & 1.2 & 1.3 & ND & ND \\
\hline Ethane & 10.3 & 11.5 & 5.4 & 6.7 & 1.3 & 2.0 & 2.4 & 2.9 & 5.3 & 6.0 & 8.2 & 9.2 \\
\hline Chlorodifluoromethane & 3.0 & 2.8 & 0.5 & 0.6 & 0.7 & ND & ND & ND & ND & ND & ND & ND \\
\hline Propylene & 2.6 & 3.2 & 2.2 & 2.4 & 0.3 & 0.4 & 0.3 & 0.4 & 1.9 & 2.2 & 2.2 & 2.6 \\
\hline Propane & 6.4 & 3.5 & 2.0 & 2.3 & ND & 0.5 & 0.7 & 0.7 & 11.2 & 7.5 & 3.0 & 3.6 \\
\hline Chloromethane & 3.1 & 4.1 & 3.7 & 4.0 & 1.8 & 3.0 & 0.1 & 0.1 & 3.8 & 4.2 & 6.1 & 7.0 \\
\hline Dichlorodifluoromethane & 2.4 & 3.3 & 2.7 & 2.9 & 0.8 & 1.3 & 0.4 & 0.8 & 2.1 & 2.8 & 2.8 & 2.1 \\
\hline Isobutane & 9.5 & 1.1 & 0.4 & 0.3 & ND & 0.3 & ND & 0.2 & 0.3 & 0.5 & 0.6 & 0.6 \\
\hline Isobutene + 1-Butene & 2.6 & 1.3 & 1.1 & 1.1 & ND & ND & ND & ND & 0.3 & 1.2 & 1.2 & 1.4 \\
\hline n-Butane & 3.8 & 0.9 & 0.9 & 1.1 & 0.3 & ND & 0.4 & 0.5 & 1.1 & 1.3 & 1.3 & 1.6 \\
\hline Methanol & 245 & 142 & 43.4 & 5.8 & 3.3 & 5.5 & ND & 10.7 & 8.9 & 9.6 & ND & ND \\
\hline Chloroethane & 0.9 & 1.1 & 0.9 & 1.0 & ND & ND & ND & ND & ND & 0.4 & 0.8 & 1.1 \\
\hline Isopentane & 11.7 & 3.6 & 0.6 & 0.6 & 0.5 & ND & 0.8 & 0.5 & 0.9 & 0.8 & 0.8 & 0.7 \\
\hline Acetone & 31.3 & 21.8 & 19.5 & 22.3 & $0.9^{\circ}$ & 1.8 & 2.8 & 0.4 & 20.7 & 18.1 & 17.1 & 18.9 \\
\hline Trichlorofluoromethane & 1.5 & 1.9 & 0.4 & 0.4 & 0.3 & 0.3 & 0.1 & 0.2 & 0.3 & 0.4 & 0.5 & 0.6 \\
\hline 1-Pentene & ND & 0.3 & ND & ND & ND & ND & ND & ND & ND & ND & 0.2 & 0.2 \\
\hline 2-Methyl-1-Butene & 0.3 & ND & ND & $\mathrm{ND}$ & ND & ND & ND & ND & ND & ND & ND & ND \\
\hline n-Pentane & 4.3 & 1.3 & 0.7 & 0.7 & 0.3 & ND & 0.5 & 0.5 & 0.9 & 0.7 & 0.6 & 0.7 \\
\hline 1,1-Dichloroethylene & 3.4 & 4.0 & 5.0 & 4.9 & 0.5 & 1.6 & 0.3 & 1.5 & 3.4 & 4.2 & 5.3 & 6.1 \\
\hline Methylene Chloride & 1.9 & 2.1 & 2.0 & 1.9 & 0.1 & 0.2 & 0.1 & 0.1 & 1.4 & 1.4 & 1.9 & 2.1 \\
\hline Neohexane & 0.2 & 0.3 & 0.2 & 0.3 & ND & ND & ND & ND & ND & ND & ND & 0.2 \\
\hline Freon 113 & 1.9 & 2.0 & 2.4 & 2.3 & 0.5 & 0.8 & 0.2 & 0.6 & 2.0 & 2.0 & 2.2 & 2.3 \\
\hline 1,1-Dichloroethane & 11.0 & 13.1 & 15.5 & 15.7 & 1.5 & 5.6 & 0.8 & 4.4 & 9.5 & 12.2 & 13.2 & 15.9 \\
\hline MTBE + Isohex + c-4-m-2-Pentene & 2.0 & 2.1 & 0.4 & 0.4 & 0.3 & 0.2 & 0.3 & 0.3 & ND & ND & ND & ND \\
\hline Butyraldehyde & ND & ND & 2.9 & 2.0 & 0.4 & ND & 0.7 & 0.8 & ND & ND & ND & ND \\
\hline 2-Butanone & 9.8 & 8.2 & 7.5 & 8.0 & 0.3 & 0.7 & 0.2 & 0.5 & ND & ND & ND & ND \\
\hline
\end{tabular}


Table 3-5

(Continued)

\begin{tabular}{|c|c|c|c|c|c|c|c|c|c|c|c|c|}
\hline \multirow{2}{*}{ Compound } & \multicolumn{2}{|c|}{$6 / 10 / 94$} & \multicolumn{2}{|c|}{$6 / 13 / 94$} & \multicolumn{2}{|c|}{$6 / 14 / 94$} & \multicolumn{2}{|c|}{$7 / 28 / 94$} & \multicolumn{2}{|c|}{$7 / 29 / 94$} & \multicolumn{2}{|c|}{$8 / 01 / 94$} \\
\hline & A & B & A & B & A & B & A & B & A & B & A & B \\
\hline 3-Methylpentane & ND & 0.4 & ND & ND & ND & ND & 0.3 & 0.2 & ND & ND & 0.6 & 1.1 \\
\hline n-Hexane & 2.1 & 1.0 & 0.5 & 0.5 & ND & ND & 0.2 & 0.2 & 0.3 & 0.3 & 0.3 & 0.3 \\
\hline Chloroform & 0.4 & 0.5 & 0.7 & 0.7 & ND & 0.1 & ND & 0.1 & 0.2 & 0.4 & 0.5 & 0.7 \\
\hline Methylcyclopentane & 1.1 & 1.1 & ND & 0.2 & ND & ND & ND & 0.2 & 0.2 & 0.3 & 0.5 & 0.6 \\
\hline 1,2-Dichloroethane & 0.3 & 0.3 & 0.4 & 0.4 & ND & 0.06 & ND & ND & ND & ND & 0.4 & 0.4 \\
\hline 1,1,1-Trichloroethane & 383 & 415 & 529 & 486 & 58.0 & 149 & 36.8 & 105 & 330 & 342 & 464 & 494 \\
\hline Benzene & 1.5 & 0.9 & 0.6 & 0.8 & 0.3 & 0.2 & 0.3 & 0.3 & 0.6 & 0.6 & 0.6 & 0.7 \\
\hline Carbon Tetrachloride & ND & ND & ND & ND & ND & ND & ND & ND & ND & ND & 0.1 & 0.1 \\
\hline 1-Butanol + Cyclohexane & 17.9 & 1.9 & 1.6 & 2.6 & 0.3 & 0.2 & ND & ND & ND & ND & 0.8 & 0.9 \\
\hline Isoheptane $+2,3$-Dimethylpentane & 1.3 & 0.5 & 0.3 & 0.3 & ND & ND & 0.4 & 0.3 & ND & 0.3 & ND & 0.6 \\
\hline 3-Methylhexane & 0.6 & 0.5 & 0.4 & 0.4 & ND & ND & 0.4 & 0.2 & ND & ND & ND & ND \\
\hline Trichloroethylene + BDCMethane & 0.6 & 0.6 & 1.4 & 1.6 & 0.04 & 0.2 & 0.06 & 0.3 & 0.5 & 0.5 & 0.7 & 0.7 \\
\hline 1,4-Dioxane + 2,2,4-TMPentane & 17.0 & 1.9 & ND & ND & ND & ND & ND & ND & ND & 0.1 & 0.4 & 0.4 \\
\hline n-Heptane & 0.8 & 0.7 & 0.7 & 0.7 & ND & ND & 0.2 & 0.2 & 0.5 & 0.5 & 0.7 & 0.8 \\
\hline Methylcyclohexane & 0.4 & ND & 0.2 & 0.3 & ND & ND & ND & ND & 0.2 & 0.2 & ND & ND \\
\hline Methylisobutylketone & 0.7 & ND & 1.7 & 2.4 & ND & ND & ND & ND & 0.8 & 1.0 & 2.8 & 3.1 \\
\hline 2,5-Dimethylhexane & ND & ND & ND & ND & ND & ND & ND & ND & ND & ND & 0.6 & 0.8 \\
\hline 2,2,3-Trimethylpentane & 1.2 & ND & ND & ND & ND & ND & ND & 0.2 & ND & ND & ND & ND \\
\hline 1,1,2-Trichloroethane & ND & ND & 0.1 & 0.07 & ND & ND & ND & ND & ND & ND & 0.04 & 0.06 \\
\hline 2,3,4-Trimethylpentane & 1.2 & ND & ND & ND & ND & ND & ND & ND & ND & ND & ND & ND \\
\hline Toluene & 10.5 & 8.0 & 6.4 & 6.8 & 0.8 & 1.5 & 0.9 & 1.2 & 4.7 & 5.0 & 5.7 & 5.6 \\
\hline Hexanal & ND & ND & 0.8 & 2.0 & ND & ND & 0.3 & 0.5 & 0.4 & 0.9 & 0.9 & 1.7 \\
\hline n-Octane & 0.6 & ND & 0.2 & 0.2 & ND & ND & 0.1 & 0.2 & ND. & ND & 0.1 & 0.2 \\
\hline Tetrachloroethylene & 2.3 & 2.7 & 2.4 & 2.9 & 0.2 & 0.7 & 0.07 & 0.4 & 1.2 & 1.7 & 2.2 & 2.8 \\
\hline Ethylbenzene & 1.1 & 0.8 & 1.0 & 1.0 & ND & ND & ND & 0.1 & 0.6 & 0.7 & 0.9 & 1.1 \\
\hline $\mathrm{p}$-Xylene + m-Xylene & 2.8 & 2.0 & 3.0 & 3.0 & 0.3 & 0.2 & 0.3 & 0.4 & 1.8 & 1.8 & 2.8 & 3.1 \\
\hline Styrene & ND & ND & 0.2 & 0.5 & ND & ND & ND & ND & ND & ND & 0.3 & 0.7 \\
\hline
\end{tabular}


Table 3-5

(Continued)

\begin{tabular}{|c|c|c|c|c|c|c|c|c|c|c|c|c|}
\hline \multirow[b]{2}{*}{ Compound } & \multicolumn{2}{|c|}{$6 / 10 / 94$} & \multicolumn{2}{|c|}{$6 / 13 / 94$} & \multicolumn{2}{|c|}{$6 / 14 / 94$} & \multicolumn{2}{|c|}{$7 / 28 / 94$} & \multicolumn{2}{|c|}{$7 / 29 / 94$} & \multicolumn{2}{|c|}{$8 / 01 / 94$} \\
\hline & A & B & A & B & A & $\mathrm{B}$ & $\mathbf{A}$ & B & A & B & A & B \\
\hline Heptanal & ND & ND & ND & 0.6 & $\mathrm{ND}$ & ND & ND & 0.1 & ND & ND & ND & ND \\
\hline o-Xylene & 0.9 & 0.6 & 0.8 & 0.9 & ND & ND & 0.1 & 0.2 & 0.6 & 0.7 & 0.9 & 1.0 \\
\hline n-Nonane & 0.1 & ND & ND & ND & ND & ND & ND & ND & ND & ND & ND & ND \\
\hline Cumene & ND & ND & ND & 0.1 & ND & ND & ND & ND & ND & ND & ND & ND \\
\hline a-Pinene + Benzaldehyde & ND & ND & 0.3 & 0.8 & ND & ND & ND & 0.1 & 0.2 & 0.2 & ND & 0.2 \\
\hline m-Ethyltoluene & 0.1 & ND & ND & 0.3 & ND & ND & ND & ND & ND & ND & ND & ND \\
\hline p-Ethyltoluene & 0.4 & ND & ND & ND & ND & ND & ND & ND & ND & ND & ND & ND \\
\hline 1,3,5-Trimethylbenzene & 0.6 & ND & ND & ND & ND & ND & ND & ND & ND & ND & ND & ND \\
\hline b-Pinene & ND & ND & ND & 0.6 & ND & ND & ND & ND & ND & ND & ND & ND \\
\hline 1,2,4-TMbenzene + t-Butylbenzene & 1.9 & 1.9 & ND & ND & ND & ND & ND & ND & ND & ND & ND & ND \\
\hline n-Decane $+p$-Dichlorobenzene & 8.0 & 1.5 & 0.3 & 0.4 & ND & ND & 0.1 & 0.2 & 0.1 & 0.2 & ND & 0.2 \\
\hline 1,2,3-Trimethylbenzene & ND & ND & ND & 1.3 & ND & ND & ND & ND & ND & ND & ND & ND \\
\hline Limonene & 0.2 & ND & 0.2 & 0.3 & ND & ND & ND & ND & 0.1 & 0.2 & 0.2 & 0.2 \\
\hline Indene & 0.2 & ND & ND & ND & ND & ND & ND & ND & ND & ND & ND & ND \\
\hline n-Butylbenzene & 0.1 & ND & 0.2 & 0.3 & ND & ND & ND & ND & 0.1 & 0.2 & 0.2 & 0.2 \\
\hline $\mathrm{n}$-Undecane & 2.5 & 0.3 & 0.3 & 0.3 & ND & ND & 0.1 & 0.2 & ND & ND & ND & 0.2 \\
\hline Naphthalene & 0.2 & ND & ND & 0.1 & ND & ND & ND & ND & ND & ND & ND & ND \\
\hline Total Unidentified VOCs & 26.8 & 19.6 & 20.9 & 20.9 & 1.1 & 3.5 & 2.2 & 5.9 & 18.2 & 20.2 & 17.8 & 24.7 \\
\hline Total Unidentified Halogenated VOCs & 8.0 & 5.8 & 8.0 & 9.2 & 0.7 & 2.8 & 2.7 & 6.0 & 7.2 & 8.2 & 8.1 & 11.4 \\
\hline TNMHC & 210 & 102 & 77.5 & 83.9 & 6.5 & 10.4 & 11.7 & 19.9 & 55.3 & 57.9 & 57.7 & 73.2 \\
\hline
\end{tabular}

ND - Not Detected

NOTE: $\quad 6 / 10 / 94$ samples collected after dome had been closed for 16 hours.

6/13/94 samples collected after dome had been closed for 64 hours.

6/14/94 samples collected with dome open.

7/28/94 samples collected with dome open.

7/29/94 samples collected after dome had been closed for 16 hours.

8/01/94 samples collected after dome had been closed for 64 hours. 


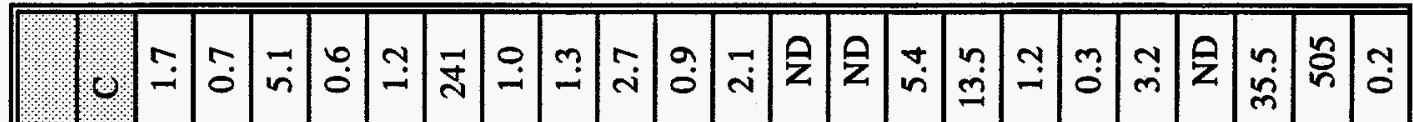



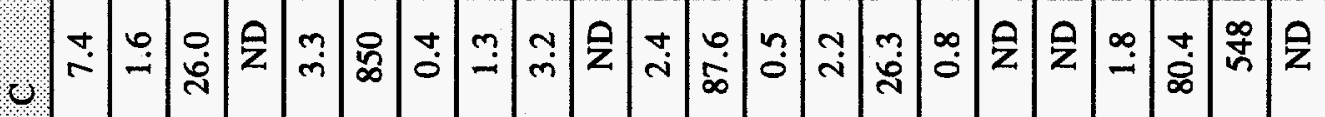

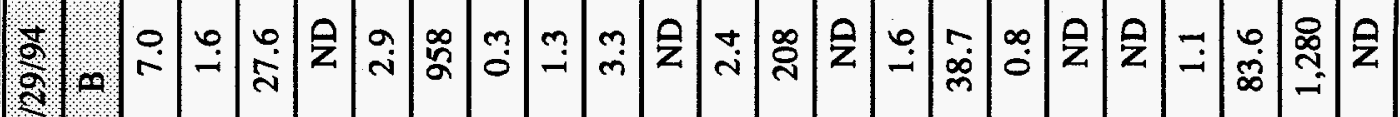

$$
\text { ব }
$$

$$
\text { 1) }
$$$$
\text { d. }
$$

$$
\text { 寉 }
$$




Table 3-6, Continued

\begin{tabular}{|c|c|c|c|c|c|c|c|c|c|}
\hline \multirow[b]{2}{*}{ Compound } & \multicolumn{3}{|c|}{$7 / 28194$} & \multicolumn{3}{|c|}{$7 / 29 / 94$} & \multicolumn{3}{|c|}{$8101 / 94$} \\
\hline & A. & B. & C. & $\mathrm{A}$ & B & C & A & $\mathrm{B}$. & C. \\
\hline Freon 113 & ND & ND & 1.0 & 15.0 & 20.6 & 38.7 & 30.9 & 57.5 & 19.0 \\
\hline 1,1-Dichloroethane & ND & ND & ND & 0.2 & 0.2 & 0.3 & 0.3 & 0.2 & 0.4 \\
\hline Cyclopentane & ND & ND & ND & 0.2 & 0.3 & 0.3 & 0.3 & 0.5 & 0.7 \\
\hline 2,3-Dimethylbutane & ND & ND & ND & ND & 0.2 & 0.2 & ND & ND & 0.3 \\
\hline MTBE + Isohex + c-4-m-2-Pentene & 0.2 & ND & ND & ND & ND & ND & ND & ND & ND \\
\hline Butyraldehyde & 0.5 & 0.4 & 0.7 & ND & ND & ND & ND & ND & ND \\
\hline 2-Butanone & 1.5 & 2.3 & 2.5 & ND & ND & ND & ND & ND & ND \\
\hline 3-Methylpentane & ND & ND & ND & ND & ND & ND & 0.8 & 1.2 & 3.3 \\
\hline c-1,2-Dichloroethylene & ND & ND & ND & 0.2 & 0.3 & ND & ND & ND & ND \\
\hline $\mathrm{n}$-Hexane & 0.2 & ND & 0.1 & 1.2 & 1.5 & 2.2 & 1.8 & 1.7 & 2.9 \\
\hline Chloroform & 0.06 & 0.1 & 0.2 & 1.9 & 2.8 & 4.8 & 3.2 & 4.7 & 4.8 \\
\hline Methylcyclopentane & ND & ND & ND & 0.5 & 0.6 & 0.9 & 0.7 & 0.6 & 1.2 \\
\hline 1,2-Dichloroethane & ND & ND & ND & 0.3 & 0.6 & 0.4 & 0.3 & 0.4 & 0.3 \\
\hline 2,4-Dimethylpentane & ND & ND & ND & ND & ND & ND & ND & ND & 0.1 \\
\hline 1,1,1-Trichloroethane & 0.4 & 0.7 & 0.8 & 4.6 & 5.5 & 7.6 & 6.3 & 8.0 & 8.2 \\
\hline Benzene & 0.3 & 0.2 & 0.2 & 0.7 & 0.8 & 1.0 & 1.0 & 1.1 & 1.3 \\
\hline Carbon Tetrachloride & 0.07 & 0.1 & 0.1 & 0.1 & 0.07 & 0.1 & 0.4 & 0.4 & 0.5 \\
\hline 1-Butanol + Cyclohexane & ND & ND & ND & 0.4 & 0.5 & 0.8 & 1.3 & 1.1 & 3.7 \\
\hline Isoheptane $+2,3$-Dimethylpentane & ND & ND & ND & 1.2 & 1.1 & 1.4 & 1.5 & 1.5 & 1.8 \\
\hline Trichloroethylene + BDCMethane & 0.4 & 0.2 & 1.2 & 10.1 & 11.5 & 15.4 & 9.8 & 12.3 & 14.4 \\
\hline 1,4-Dioxane + 2,2,4-TMPentane & 0.2 & 0.5 & 0.5 & ND & ND & ND & ND & ND & 0.7 \\
\hline n-Heptane & 0.1 & 0.2 & 0.2 & 1.8 & 1.9 & 2.4 & 2.1 & 2.1 & 2.5 \\
\hline Methylcyclohexane & 0.1 & 0.2 & 0.3 & 2.0 & $2.3^{\circ}$ & 2.9 & ND & ND & 0.3 \\
\hline
\end{tabular}


Table 3-6, Continued

\begin{tabular}{|c|c|c|c|c|c|c|c|c|c|}
\hline \multirow{2}{*}{ Compound } & \multicolumn{3}{|c|}{$7 / 28 \% 94$} & \multicolumn{3}{|c|}{$729 / 94$} & \multicolumn{3}{|c|}{$8 / 01 / 94$} \\
\hline & $\mathrm{A}$ & B & 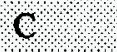 & $\mathrm{A}$ & B & 8 & A & B. & c. \\
\hline Methylisobutylketone & ND & ND & ND & 1.3 & 3.1 & 1.6 & 4.6 & 6.3 & 5.4 \\
\hline 2,5-Dimethylhexane & ND & ND & ND & 0.2 & 0.2 & 0.2 & 0.2 & ND & 0.3 \\
\hline 2,2,3-Trimethylpentane & ND & ND & ND & 0.1 & 0.1 & 0.2 & 0.2 & ND & 0.3 \\
\hline Toluene & 1.1 & 1.7 & 2.3 & 15.5 & 21.2 & 19.1 & 18.1 & 22.8 & 22.1 \\
\hline 3-Methylheptane & ND & ND & ND & 1.1 & 1.1 & 1.4 & 1.0 & 1.0 & 1.3 \\
\hline Hexanal & 0.2 & 0.2 & 0.2 & 1.8 & 1.9 & 2.4 & 3.2 & 1.5 & 3.2 \\
\hline 1-Octene & ND & ND & ND & ND & ND & ND & ND & 0.1 & $\overline{\mathrm{ND}}$ \\
\hline n-Octane & 0.2 & 0.3 & 0.5 & 3.4 & 3.6 & 3.9 & 4.0 & 4.0 & 4.8 \\
\hline Tetrachloroethylene & ND & ND & ND & 0.2 & 0.2 & 0.2 & 0.3 & 0.4 & $\overline{0.4}$ \\
\hline Ethylbenzene & ND & 0.2 & 0.3 & 2.0 & 5.4 & 3.0 & 2.5 & 6.0 & 3.3 \\
\hline p-Xylene $+\mathrm{m}$-Xylene & 0.2 & 0.5 & 0.7 & 4.5 & 12.7 & 6.9 & 5.9 & 13.7 & 7.3 \\
\hline Bromoform & ND & ND & ND & ND & ND & ND & 0.1 & ND & 0.08 \\
\hline Styrene & ND & ND & ND & ND & ND & ND & 0.8 & 0.3 & $\overline{0.6}$ \\
\hline o-Xylene & 0.1 & 0.2 & 0.2 & 1.0 & 2.5 & 5.0 & 1.6 & 3.1 & 1.9 \\
\hline n-Nonane & ND & ND & 0.09 & 0.5 & 0.6 & 0.9 & 0.7 & 0.8 & $\overline{1.2}$ \\
\hline a-Pinene + Benzaldehyde & ND & 0.2 & 0.2 & 0.6 & 0.9 & 1.1 & 0.9 & 0.7 & 1.2 \\
\hline o-Chlorotoluene & ND & ND & ND & ND & ND & ND & 0.2 & ND & 0.3 \\
\hline n-Propylbenzene & ND & ND & ND & ND & 0.1 & 0.2 & ND & 0.2 & $\overline{\text { ND }}$ \\
\hline m-Ethyltoluene & ND & ND & ND & 0.1 & 0.2 & 0.3 & 0.3 & 0.3 & $\overline{0.5}$ \\
\hline p-Ethyltoluene & ND & ND & ND & ND & 0.3 & 0.3 & 0.3 & 0.4 & $\overline{0.6}$ \\
\hline 1,3,5-Trimethylbenzene & ND & ND & ND & ND & 0.2 & 0.2 & 0.3 & 0.4 & 0.7 \\
\hline o-Ethyltoluene & ND & ND & ND & 0.1 & 0.2 & 0.2 & ND & ND & 0.1 \\
\hline b-Pinene & ND & ND & ND & ND & ND & ND & 0.3 & 0.1 & 0.2 \\
\hline
\end{tabular}


Table 3-6, Continued

\begin{tabular}{|c|c|c|c|c|c|c|c|c|c|}
\hline \multirow[b]{2}{*}{ Compound } & \multicolumn{3}{|c|}{$7 / 28 / 94$} & \multicolumn{3}{|c|}{$7 / 29 / 94$} & \multicolumn{3}{|c|}{$8 / 01 / 94$} \\
\hline & A & B & c & A & B. & C & A & B & c. \\
\hline 1,2,4-TMBenzene + $t$-Butylbenzene & ND & ND & ND & 0.3 & 0.5 & 0.7 & 0.7 & 0.7 & 1.1 \\
\hline n-Decane + p-Dichlorobenzene & ND & 0.2 & 0.2 & 0.6 & 0.9 & 1.0 & 0.9 & 1.0 & 2.6 \\
\hline 1,2,3-Trimethylbenzene & ND & ND & ND & ND & 0.1 & 0.2 & 0.3 & 0.3 & 0.5 \\
\hline Limonene & ND & ND & ND & ND & 0.2 & 0.2 & 0.2 & ND & 0.4 \\
\hline n-Butylbenzene & ND & ND & ND & ND & 0.1 & 0.1 & 0.09 & 0.1 & 0.2 \\
\hline p-Diethylbenzene & ND & ND & ND & ND & 0.09 & 0.1 & 0.2 & 0.2 & 0.3 \\
\hline n-Undecane & 0.2 & 0.3 & 0.2 & 0.3 & 0.4 & 0.5 & 0.6 & 0.7 & 1.9 \\
\hline Naphthalene & ND & ND & ND & ND & ND & ND & ND & ND & 0.2 \\
\hline Total Unidentified VOCs & 1.8 & 9.5 & 3.6 & 26.9 & 43.7 & 34.4 & 27.3 & 38.8 & 37.2 \\
\hline Total Unidentified Halogenated VOCs & ND & ND & ND & 0.7 & 1.9 & 0.3 & 0.4 & 0.5 & 1.0 \\
\hline TNMHC & 87.9 & 113 & 146 & 574 & 679 & 584 & 275 & 344 & 279 \\
\hline
\end{tabular}

$\mathrm{ND}=$ Not Detected

NOTE: 7/28/94 samples collected with dome open.

$7 / 29 / 94$ samples collected after dome had been closed for 16 hours.

8/01/94 samples collected after dome had been closed for 64 hours.

See Figure 3-1 for relative locations of sampling points A, B, and C. 
Table 3-7

Summary of Dome 153 VOC Results

\begin{tabular}{|c|c|c|c|c|c|c|c|c|c|c|c|c|c|c|c|c|c|c|}
\hline \multirow{2}{*}{ Compound } & \multicolumn{3}{|c|}{$6 / 10 / 94$} & \multicolumn{3}{|c|}{$6 / 13 / 94$} & \multicolumn{3}{|c|}{$6 / 14 / 94$} & \multicolumn{3}{|c|}{$7 / 28 / 94$} & \multicolumn{3}{|c|}{$7 / 29 / 94$} & \multicolumn{3}{|c|}{$8 / 01 / 94$} \\
\hline & A & B & C & A & B & $\mathrm{C}$ & A & B & C & A & B & C & A & B & C & A & B & $\mathrm{C}$ \\
\hline Ethylene & 2.0 & 2.2 & 2.6 & 1.6 & 1.7 & 2.1 & 0.5 & 0.5 & 0.6 & 0.6 & 0.6 & 0.6 & 1.6 & 1.9 & 1.9 & 1.4 & 1.9 & 1.5 \\
\hline Acetylene & ND & ND & ND & ND & ND & ND & ND & ND & ND & ND & ND & ND & 0.3 & ND & ND & ND & ND & ND \\
\hline Ethane & 5.1 & 5.1 & 4.6 & 2.3 & 2.2 & 2.2 & 1.4 & 0.9 & 1.2 & 1.8 & 2.3 & 2.0 & 2.6 & 3.0 & 3.0 & 3.0 & 4.3 & 4.4 \\
\hline Chlorodifluoromethane & 0.7 & 1.2 & 0.5 & 0.7 & 0.6 & 0.4 & 0.6 & 0.5 & 0.6 & ND & ND & ND & ND & ND & ND & 0.4 & ND & ND \\
\hline Propylene & 1.1 & 1.1 & 1.3 & 1.0 & 1.0 & 1.1 & 0.3 & 0.3 & 0.3 & 0.2 & 0.3 & 0.3 & 1.0 & 1.1 & 1.0 & 1.2 & 1.5 & 0.9 \\
\hline Propane & 3.4 & 3.5 & 3.4 & 2.4 & 2.0 & 2.1 & 0.5 & 0.3 & 0.8 & 0.5 & 0.9 & 0.6 & 2.2 & 2.5 & 2.8 & 3.4 & 3.7 & 3.6 \\
\hline Chloromethane & 2.0 & 2.1 & 2.3 & 2.1 & 1.8 & 2.0 & 1.1 & 1.3 & 1.7 & ND & ND & ND & 2.5 & 2.3 & 2.0 & 2.9 & 4.0 & 3.3 \\
\hline Dichlorodifluoromethane & 0.8 & 0.8 & 0.8 & 0.8 & 0.9 & 0.9 & 0.6 & 0.6 & 0.8 & 0.3 & 0.3 & 0.3 & 0.7 & 0.7 & 0.8 & 0.9 & 1.1 & 1.1 \\
\hline Isobutane & 0.6 & 0.6 & 0.6 & 1.0 & 0.4 & 0.5 & ND & ND & 1.0 & ND & ND & ND & 1.1 & 1.7 & 1.2 & 1.7 & 1.5 & 1.7 \\
\hline Isobutene + 1-Butene & 0.6 & 0.6 & 0.7 & 0.5 & 0.6 & 0.6 & $\mathrm{ND}$ & ND & ND & ND & ND & ND & 0.5 & 0.6 & 0.7 & 0.9 & 2.1 & 0.7 \\
\hline n-Butane & 1.2 & 0.8 & 0.7 & 0.7 & 0.7 & 0.7 & 0.3 & 13.0 & 0.4 & ND & 0.4 & ND & 0.7 & 1.5 & 1.5 & 1.0 & 1.0 & 0.9 \\
\hline Methanol & 29.5 & 36.3 & 129.0 & 42.3 & 28.6 & 33.2 & 3.6 & 2.4 & 22.6 & ND & 1.2 & ND & ND & 42.0 & ND & ND & ND & ND \\
\hline Bromomethane & 40.3 & 43.0 & 56.5 & 47.0 & 39.8 & 53.6 & 2.8 & ND & 4.6 & ND & ND & ND & 17.4 & 23.7 & 40.6 & 43.4 & 30.6 & 46.6 \\
\hline Chloroethane & 0.2 & 0.2 & 0.2 & 0.3 & 0.2 & 0.2 & ND & ND & ND & ND & ND & ND & 0.3 & 0.4 & ND & 0.4 & 0.7 & ND \\
\hline Isopentane & 1.3 & 0.7 & 0.7 & 1.3 & 0.8 & 0.7 & 0.3 & 0.6 & 3.7 & 0.4 & 0.4 & 0.4 & 0.7 & 0.7 & 0.7 & 0.7 & 0.8 & 0.7 \\
\hline Acetone & 3.1 & 2.9 & 3.4 & 3.4 & 2.8 & 3.6 & 1.4 & 2.9 & ND & 2.5 & 2.4 & 2.6 & 5.6 & 8.2 & 7.0 & 5.9 & 54.4 & 5.6 \\
\hline Trichlorofluoromethane & 1.0 & 1.1 & 1.2 & 1.1 & 1.1 & 1.3 & 0.3 & 0.3 & 2.1 & 0.1 & 0.1 & 0.2 & 1.4 & 1.7 & 2.1 & 1.3 & 1.6 & 1.8 \\
\hline n-Pentane & 1.3 & 1.4 & 1.1 & 1.2 & 1.2 & 1.2 & 0.3 & 0.4 & 0.8 & 0.3 & 0.3 & 0.3 & 0.9 & 1.1 & 1.0 & 0.9 & 1.0 & 1.0 \\
\hline 1,1-Dichloroethylene & ND & ND & ND & ND & ND & ND & ND & ND & ND & ND & ND & ND & 0.06 & 0.1 & 0.07 & 0.9 & 0.9 & 0.9 \\
\hline Methylene Chloride & 2.4 & 2.4 & 3.1 & 2.4 & 2.1 & 2.7 & 0.1 & 0.1 & 0.2 & ND & 0.06 & 0.8 & 2.3 & 3.4 & 3.1 & 2.5 & 3.5 & 3.3 \\
\hline Freon 113 & 10.7 & 9.8 & 7.1 & 8.6 & 8.6 & 7.1 & 0.6 & 1.1 & 1.1 & 0.1 & 0.2 & 0.2 & 12.0 & 13.2 & 8.6 & 8.7 & 9.7 & 7.5 \\
\hline 1,1-Dichloroethane & 0.1 & 0.1 & 0.1 & 0.1 & 0.1 & 0.1 & ND & ND & ND & ND & ND & ND & 0.2 & 0.4 & 0.2 & 0.2 & 0.4 & 0.3 \\
\hline Cyclopentane & ND & ND & ND & ND & ND & ND & ND & ND & ND & ND & ND & ND & ND & 0.2 & 0.2 & ND & ND & ND \\
\hline 2,3-Dimethylbutane & ND & ND & ND & ND & ND & ND & ND & ND & ND & ND & ND & ND & ND & ND & ND & ND & ND & 0.2 \\
\hline $\begin{array}{l}\text { MTBE + Isohex + } \\
\text { c-4-m-2-Pentene }\end{array}$ & 0.7 & 0.6 & 0.6 & 0.5 & 0.6 & 0.5 & 0.4 & 0.7 & 0.8 & 0.2 & 0.3 & 0.2 & ND & ND & ND & ND & ND & ND \\
\hline
\end{tabular}




\begin{tabular}{|c|c|c|c|c|c|c|c|c|c|c|c|c|c|c|c|c|c|c|}
\hline \multirow[b]{2}{*}{ Compound } & \multicolumn{3}{|c|}{$6 / 10 / 94$} & \multicolumn{3}{|c|}{$6 / 13 / 94$} & \multicolumn{3}{|c|}{ 6/14/94 } & \multicolumn{3}{|c|}{ 7/28/94 } & \multicolumn{3}{|c|}{ 7/29/94 } & \multicolumn{3}{|c|}{$8 / 01 / 94$} \\
\hline & A & B & c & A & B & C & A & B & c & A & B & C & A & B & C & A & B & c \\
\hline 2-Butanone & 2.3 & 2.8 & 3.4 & 2.8 & 2.4 & 2.7 & ND & ND & ND & ND & ND & ND & ND & ND & ND & ND & ND & ND \\
\hline 3-Methylpentane & 0.4 & 0.4 & 0.3 & 0.4 & 0.4 & 0.3 & ND & 0.2 & ND & ND & 0.1 & ND & ND & ND & ND & 0.5 & ND & 0.7 \\
\hline n-Hexane & 0.6 & 0.6 & 0.5 & 0.7 & 0.6 & 0.6 & ND & ND & 0.5 & ND & 0.2 & ND & 0.4 & 0.5 & 0.4 & 0.4 & 0.4 & 0.4 \\
\hline Chloroform & ND & ND & ND & ND & ND & ND & ND & ND & ND & ND & ND & ND & ND & ND & ND & 0.04 & 0.04 & ND \\
\hline Methylcyclopentane & ND & 0.3 & 0.2 & 0.5 & 0.4 & 0.4 & ND & ND & 0.2 & ND & ND & ND & 0.2 & 0.3 & 0.2 & 0.2 & ND & ND \\
\hline 1,1,1-Trichloroethane & 61.8 & 75.4 & 72.2 & 54.7 & 60.0 & 66.5 & 5.6 & 5.5 & 11.2 & 1.6 & 2.5 & 2.5 & 19.4 & 29.1 & 19.0 & 19.2 & 25.6 & 21.6 \\
\hline Benzene & 0.6 & 0.6 & 0.6 & 0.6 & 0.5 & 0.5 & 0.3 & 0.3 & 3.0 & 0.2 & 0.2 & 0.2 & 0.3 & 0.3 & 0.3 & 0.5 & 0.4 & 0.4 \\
\hline Carbon Tetrachloride & ND & ND & ND & ND & ND & ND & 0.05 & 0.03 & 0.1 & 0.07 & 0.1 & 0.1 & ND & $\mathrm{ND}$ & ND & 0.2 & 0.3 & 0.2 \\
\hline 1-butanol + Cyclohexane & 0.5 & $\mathrm{ND}$ & 0.5 & 1.9 & 0.8 & 0.9 & 0.3 & 3.0 & 0.8 & ND & ND & ND & ND & ND & ND & 0.4 & 0.9 & 0.5 \\
\hline $\begin{array}{l}\text { Isoheptane }+ \\
\text { 2,3-Dimethylpentane }\end{array}$ & ND & ND & ND & 0.4 & 0.3 & 0.3 & ND & ND & ND & ND & 0.1 & ND & 0.2 & 0.2 & 0.2 & 0.3 & 0.8 & ND \\
\hline 3-Methylhexane & 0.3 & 0.4 & 0.3 & 0.3 & 0.4 & 0.4 & $\mathrm{ND}$ & ND & ND & 0.1 & ND & ND & ND & ND & ND & ND & ND & ND \\
\hline $\begin{array}{l}\text { Trichloroethylene + } \\
\text { BDCmethane }\end{array}$ & 0.5 & 0.6 & 0.6 & 0.4 & 0.5 & 0.5 & ND & ND & 0.05 & ND & ND & ND & 0.2 & 0.3 & 0.2 & 0.2 & 0.2 & 0.2 \\
\hline $\begin{array}{l}\text { 1,4-Dioxane }+ \\
\text { 2,2,4-TMPentane }\end{array}$ & 0.5 & 0.6 & 0.5 & 1.2 & 5.0 & 0.4 & ND & ND & ND & ND & ND & ND & ND & ND & ND & 0.2 & ND & ND \\
\hline $\mathrm{n}$-Heptane & 0.5 & 0.5 & 0.4 & 0.4 & 0.4 & 0.4 & ND & ND & 0.4 & ND & ND & ND & 0.4 & 0.4 & 0.4 & 0.4 & 0.4 & 0.4 \\
\hline Methylcyclohe xane & 0.5 & 0.5 & 0.4 & 0.4 & 0.4 & 0.4 & ND & ND & 0.4 & ND & ND & ND & 0.2 & 0.3 & 0.3 & 0.3 & ND & ND \\
\hline Methylisobutylketone & ND & ND & ND & ND & ND & ND & ND & ND & ND & ND & ND & ND & ND & ND & ND & ND & 0.7 & 0.3 \\
\hline Toluene & 3.3 & 3.4 & 2.7 & 3.5 & 3.1 & 2.8 & 0.5 & 0.4 & 4.2 & 0.4 & 0.4 & 0.4 & 2.2 & 2.4 & 2.1 & 2.3 & 2.3 & 2.0 \\
\hline Hexanal & ND & ND & ND & 0.7 & 0.3 & 0.3 & ND & ND & ND & 0.2 & 0.3 & 0.4 & 0.6 & 0.8 & 0.6 & 2.3 & 5.0 & 2.2 \\
\hline n-Octane & ND & 0.2 & ND & 0.2 & 0.2 & 0.2 & ND & ND & ND & ND & ND & ND & ND & 0.3 & 0.2 & 0.1 & 0.2 & 0.1 \\
\hline Tetrachloroethylene & 0.6 & 0.4 & 0.3 & 0.4 & 0.5 & 0.3 & ND & 0.03 & 0.05 & ND & ND & ND & 0.3 & 0.3 & 0.2 & 0.4 & 0.5 & 0.3 \\
\hline Ethylbenzene & 0.3 & 0.3 & 0.3 & 0.4 & 0.3 & 0.3 & ND & $\mathrm{ND}$ & ND & $\mathrm{ND}$ & ND & ND & 0.2 & 0.3 & 0.3 & 0.3 & 0.3 & 0.3 \\
\hline $\mathrm{p}$-Xylene $+\mathrm{m}$-Xylene & 0.6 & 0.6 & 0.5 & 0.8 & 0.8 & 0.7 & ND & ND & 0.2 & 0.1 & 0.1 & 0.1 & 0.4 & 0.5 & 0.5 & 0.4 & 0.5 & 0.4 \\
\hline Styrene & ND & ND & ND & 1.5 & 0.7 & 1.1 & ND & ND & ND & ND & ND & ND & 1.3 & 1.5 & 1.2 & 3.1 & 5.2 & 3.6 \\
\hline o-Xylene & 0.2 & 0.2 & 0.2 & 0.4 & 0.3 & 0.3 & ND & ND & ND & ND & ND & ND & 0.2 & 0.3 & 0.3 & 0.3 & 0.3 & 0.2 \\
\hline a-Pinene + Benzaldehyde & ND & ND & ND & 0.6 & 0.3 & 0.3 & $\mathrm{ND}$ & $\mathrm{ND}$ & ND & ND & 0.1 & 0.1 & 0.5 & 0.7 & 0.4 & 1.0 & 1.3 & 1.1 \\
\hline m-Ethyltoluene & ND & ND & ND & 0.2 & 0.2 & 0.1 & ND & $\mathrm{ND}$ & ND & ND & ND & ND & ND & ND & ND & ND & 0.2 & ND \\
\hline
\end{tabular}


Table 3-7, Continued

\begin{tabular}{|c|c|c|c|c|c|c|c|c|c|c|c|c|c|c|c|c|c|c|}
\hline \multirow[b]{2}{*}{ Compound } & \multicolumn{3}{|c|}{$6 / 10 / 94$} & \multicolumn{3}{|c|}{$6 / 13 / 94$} & \multicolumn{3}{|c|}{$6 / 14 / 94$} & \multicolumn{3}{|c|}{$7 / 28 / 94$} & \multicolumn{3}{|c|}{ 7/29/94 } & \multicolumn{3}{|c|}{ 8/01/94 } \\
\hline & A & B & c & $\mathbf{A}$ & B & c & A & B & c & $\mathbf{A}$ & B & c & $\mathbf{A}$ & B & c & $\mathbf{A}$ & B & C \\
\hline p-Ethyltoluene & ND & ND & ND & 0.2 & 0.2 & ND & ND & ND & ND & ND & ND & ND & ND & ND & ND & ND & ND & ND \\
\hline 1,3,5-Trimethylbenzene & ND & ND & ND & 0.2 & 0.2 & 0.2 & ND & ND & ND & ND & ND & ND & ND & ND & ND & ND & ND & ND \\
\hline b-Pinene & ND & ND & ND & ND & ND & ND & ND & ND & ND & ND & ND & ND & ND & ND & ND & 0.2 & 0.9 & ND \\
\hline $\begin{array}{l}\text { 1,2,4-TMbenzene + } \\
\text { t-Butylbenzene }\end{array}$ & ND & 0.7 & ND & 0.3 & 0.2 & 0.2 & ND & ND & ND & ND & ND & ND & ND & 0.09 & ND & 0.1 & 0.1 & 0.1 \\
\hline n-Decane $+p$-Dichlorobenzene & 0.2 & 0.3 & 0.5 & 0.7 & 0.3 & 0.2 & ND & ND & ND & ND & 0.2 & ND & ND & 0.1 & ND & ND & ND & ND \\
\hline 1,2,3-Trimethylbenzene & ND & ND & ND & 0.2 & 0.2 & 0.1 & ND & ND & ND & ND & ND & ND & ND & ND & ND & 0.1 & 0.2 & 0.1 \\
\hline $\mathrm{n}$-Undecane & 0.1 & ND & ND & 0.5 & 0.3 & 0.3 & ND & ND & 0.1 & 0.1 & 0.2 & 0.1 & 0.08 & ND & 0.09 & 0.1 & 0.2 & 0.1 \\
\hline Total Unidentified VOCs & 3.3 & 3.5 & 3.8 & 5.7 & 5.2 & 4.0 & 0.7 & 2.2 & 1.7 & 1.5 & 2.3 & 2.5 & 6.5 & 9.1 & 9.6 & 7.1 & 22.9 & 5.8 \\
\hline Unidentified Halogenated VOC & 1.1 & 1.2 & 1.1 & 1.1 & 1.1 & 1.1 & 0.2 & 0.3 & 0.2 & 0.1 & 0.2 & 0.3 & 0.2 & 0.2 & 2.1 & 0.3 & 0.5 & 0.3 \\
\hline TNMHC & 30.2 & 32.2 & 46.3 & 44.6 & 35.1 & 34.3 & 5.5 & 12.6 & 23.0 & 6.4 & 9.6 & 8.0 & 23.4 & 38.1 & 29.2 & 32.6 & 81.4 & 30.3 \\
\hline
\end{tabular}

NOTE: $6 / 10 / 94$ samples collected after dome had been closed for 16 hours. 6/13/94 samples collected after dome had been closed for 64 hours. 6/14/94 samples collected with dome open.

7/28/94 samples collected with dome open.

7/29/94 samples collected after dome had been closed for 16 hours. 8/01/94 samples collected after dome had been closed for 64 hours.

See Figure 3-1 for locations of sampling points 
Table 3-8

Summary of VOC Sampling Variability (Dome Study Only)

\begin{tabular}{|c|c|c|c|c|c|c|c|c|c|c|}
\hline \multirow{3}{*}{ Compound } & \multicolumn{9}{|c|}{ Date Sampled } & \multirow{3}{*}{$\begin{array}{l}\text { Pooled } \\
\text { CV } \\
(\%)\end{array}$} \\
\hline & \multicolumn{3}{|c|}{$7 / 28 / 94$} & \multicolumn{3}{|c|}{$7 / 29 / 94$} & \multicolumn{3}{|c|}{$8 / 01 / 94$} & \\
\hline & A & B & CV (\%) & A & B & $\operatorname{cv}(\%)$ & A & B. & $\mathrm{CV}(\%)$ & \\
\hline Ethylene & 8.2 & 8.7 & 4.2 & 7.0 & 6.7 & 3.1 & 2.2 & 2.2 & 0.0 & 1.5 \\
\hline Acetylene & 2.5 & 3.0 & 12.9 & 1.6 & 1.7 & 4.3 & 0.7 & 0.7 & 0.0 & 3.9 \\
\hline Ethane & 8.6 & 8.7 & 0.8 & 27.6 & 26.5 & 2.9 & 4.1 & 5.2 & 16.7 & 4.9 \\
\hline Chlorodifluoromethane & ND & ND & NC & ND & ND & NC & 0.6 & 0.7 & 10.9 & 5.4 \\
\hline Propylene & 2.7 & 2.8 & 2.6 & 2.9 & 3.2 & 7.0 & 1.4 & 1.3 & 5.2 & 2.6 \\
\hline -Propane & 189 & 192 & 1.1 & 958 & 949 & 0.7 & 303 & 269 & 8.4 & 2.5 \\
\hline Chloromethane & 0.4 & 0.2 & 47.1 & 0.3 & 0.4 & 20.2 & 0.9 & 0.8 & 8.3 & 15.0 \\
\hline Dichlorodifluoromethane & 0.5 & 0.4 & 15.7 & 1.3 & 1.3 & 0.0 & 1.9 & 0.8 & 57.6 & 17.2 \\
\hline Isobutane & 0.7 & 0.8 & 9.4 & 3.3 & 3.5 & 4.2 & 1.7 & 2.0 & 11.5 & 4.5 \\
\hline Isobutene +1 -Butene & ND & ND & $\mathrm{NC}$ & ND & 0.7 & $\mathrm{NC}$ & 0.6 & 0.7 & 10.9 & 5.4 \\
\hline n-Butane & 0.3 & 0.7 & 56.6 & 2.4 & 2.3 & 3.0 & 1.9 & 1.9 & 0.0 & 16.4 \\
\hline Methanol & 8.6 & ND & NC & 208 & 230 & 7.1 & 5.2 & 14.7 & 67.5 & 19.6 \\
\hline Ethanol + Acetonitrile & ND & ND & $\mathrm{NC}$ & ND & ND & $\mathrm{NC}$ & ND & ND & NC & $\mathrm{NC}$ \\
\hline Isopentane & 0.3 & 0.3 & 0.0 & 1.6 & 1.5 & 4.6 & 1.6 & 2.3 & 25.4 & 7.4 \\
\hline Acetone & 3.1 & 3.1 & 0.0 & 38.7 & 62.0 & 32.7 & 47.9 & 40.1 & 12.5 & 10.1 \\
\hline Trichlorofluoromethane & 0.3 & 0.2 & 28.3 & 0.8 & 0.8 & 0.0 & 1.0 & 1.1 & 6.7 & 8.4 \\
\hline 1-Pentene & ND & ND & NC & ND & ND & NC & ND & ND & NC & NC \\
\hline lodomethane & ND & ND & $\mathrm{NC}$ & ND & ND & NC & ND & 3.3 & NC & NC \\
\hline n-Pentane & 0.2 & 0.2 & 0.0 & 1.1 & 1.2 & 6.1 & 3.6 & ND & NC & 1.8 \\
\hline Diethyl Ether + 2-Propanol & ND & ND & NC & 83.6 & 136 & 33.7 & 65.3 & 82.5 & 16.5 & 13.3 \\
\hline Methylene Chloride & 126 & 59.2 & 51.0 & 1280 & 1210 & 4.0 & 1450 & 1330 & 6.1 & 14.9 \\
\hline
\end{tabular}


Table 3-8, Continued

\begin{tabular}{|c|c|c|c|c|c|c|c|c|c|c|}
\hline \multirow{3}{*}{ Compound } & \multicolumn{9}{|c|}{ Date Sampled } & \multirow{3}{*}{$\begin{array}{l}\text { Pooled } \\
\text { CV } \\
(\%)\end{array}$} \\
\hline & \multicolumn{3}{|c|}{$7 / 28 / 94$} & \multicolumn{3}{|c|}{$7 / 29 / 94$} & \multicolumn{3}{|c|}{$8 / 01 / 94$} & \\
\hline & A. & B & $\mathrm{CV}(\%)$ & A & B & $\mathrm{CV}(\%)$ & A. & B & $\mathrm{cV}(\%)$ & \\
\hline Neohexane & ND & ND & NC & ND & ND & NC & ND & ND & NC & NC \\
\hline Freon 113 & ND & ND & NC & 20.6 & 20.2 & 1.4 & 57.5 & 57.0 & 0.6 & 0.5 \\
\hline 1,1-Dichloroethane & ND & ND & $\mathrm{NC}$ & 0.2 & 0.3 & 28.3 & 0.2 & 0.3 & 28.3 & 14.1 \\
\hline Cyclopentane & ND & ND & NC & 0.3 & 0.3 & 0.0 & 0.5 & 0.5 & 0.0 & 0.0 \\
\hline 2,3-Dimethylbutane & ND & ND & NC & 0.2 & 0.2 & 0.0 & ND & 0.2 & NC & 0.0 \\
\hline MTBE + Isohex + c-4-m-2-Pentene & ND & 0.2 & NC & ND & ND & NC & ND & ND & NC & NC \\
\hline Butyraldehyde & 0.4 & 0.5 & 15.7 & ND & ND & NC & ND & ND & NC & 7.9 \\
\hline 2-Butanone & 2.3 & 1.8 & 17.2 & ND & ND & NC & ND & ND & NC & 8.6 \\
\hline 3-Methylpentane & ND & ND & NC & ND & ND & $\mathrm{NC}$ & 1.2 & 1.3 & 5.7 & 2.8 \\
\hline c-1,2-Dichloroethylene & ND & ND & $\mathrm{NC}$ & 0.3 & ND & NC & ND & ND & NC & $\mathrm{NC}$ \\
\hline n-Hexane & ND & ND & NC & 1.5 & 1.6 & 4.6 & 1.7 & 2.2 & 18.1 & 6.6 \\
\hline Chloroform & 0.1 & 0.09 & 7.4 & 2.8 & 3.1 & 7.2 & 4.7 & 5.9 & 16.0 & 5.5 \\
\hline Methylcyclopentane & ND & ND & NC & 0.6 & 1.0 & 35.4 & 0.6 & 0.6 & 0.0 & 12.5 \\
\hline 1,2-Dichloroethane & ND & ND & $\mathrm{NC}$ & 0.6 & 0.2 & 70.7 & 0.4 & 0.8 & 47.1 & 30.0 \\
\hline 2,4-Dimethylpentane & ND & ND & NC & ND & ND & $\mathrm{NC}$ & ND & ND & NC & $\mathrm{NC}$ \\
\hline 1,1,1-Trichloroethane & 0.7 & 0.8 & 9.4 & 5.5 & 6.0 & 6.1 & 8.0 & 9.6 & 12.9 & 4.9 \\
\hline Benzene & 0.2 & 0.2 & 0.0 & 0.8 & 0.9 & 8.3 & 1.1 & 1.1 & 0.0 & 2.4 \\
\hline Carbon Tetrachloride & 0.1 & 0.08 & 15.7 & 0.07 & 0.07 & $\mathrm{NC}$ & 0.4 & 0.4 & 0.0 & 4.5 \\
\hline 1-Butanol + Cyclohexane & ND & ND & NC & 0.5 & 1.1 & 53.0 & 1.1 & 0.7 & 31.4 & 21.8 \\
\hline Isoheptane $+2,3$-Dimethylpentane & ND & 0.1 & NC & 1.1 & 1.2 & 6.1 & 1.5 & 1.5 & 0.0 & 2.2 \\
\hline Trichloroethylene + BDCMethane & 0.2 & 0.4 & 47.1 & 11.5 & 12.4 & 5.3 & 12.3 & 11.8 & 2.9 & 13.7 \\
\hline 1,4-Dioxane + 2,2,4-TMPentane & 0.5 & 0.2 & 60.6 & ND & ND & NC & ND & ND & NC & 30.3 \\
\hline
\end{tabular}


Table 3-8, Continued

\begin{tabular}{|c|c|c|c|c|c|c|c|c|c|c|}
\hline \multirow{3}{*}{ Compound } & \multicolumn{9}{|c|}{ Date Sampled } & \multirow{3}{*}{$\begin{array}{l}\text { Pooled } \\
\text { cV } \\
(\%) \text {. }\end{array}$} \\
\hline & \multicolumn{3}{|c|}{$7 / 28 / 94$} & \multicolumn{3}{|c|}{$7 / 29 / 94$} & \multicolumn{3}{|c|}{$8 / 01 / 94$} & \\
\hline & A & B. & $\mathrm{CV}(\%)$ & A. & B & CV (\%) & A. & B & $\mathrm{CV}(\%)$ & \\
\hline n-Heptane & 0.2 & 0.2 & 0.0 & 1.9 & 1.8 & 3.8 & 2.1 & 2.2 & 3.3 & 1.5 \\
\hline Methylcyclohexane & 0.2 & 0.2 & 0.0 & 2.3 & 1.7 & 21.2 & ND & ND & NC & 7.5 \\
\hline Methylisobutylketone & ND & ND & NC & 3.1 & 4.2 & 21.3 & 6.3 & 6.2 & 1.1 & 7.5 \\
\hline 2,5-Dimethylhexane & ND & ND & NC & 0.2 & 0.2 & 0.0 & ND & 0.2 & NC & 0.0 \\
\hline 2,2,3-Trimethylpentane & ND & ND & NC & 0.1 & 0.1 & 0.0 & ND & 0.1 & NC & 0.0 \\
\hline Toluene & 1.7 & 1.5 & 8.8 & 21.2 & 20.7 & 1.7 & 22.8 & 22.9 & 0.3 & 2.6 \\
\hline 3-Methylheptane & ND & ND & NC & 1.1 & 1.1 & 0.0 & 1.0 & 1.1 & 6.7 & 2.4 \\
\hline Hexanal & 0.2 & 0.2 & 0.0 & 1.9 & 1.8 & 3.8 & 1.5 & 2.3 & 29.8 & 8.7 \\
\hline 1-Octene & ND & ND & NC & ND & ND & NC & 0.1 & ND & NC & $\mathrm{NC}$ \\
\hline n-Octane & 0.3 & 0.3 & 0.0 & 3.6 & 3.2 & 8.3 & 4.0 & 4.1 & 1.7 & 2.5 \\
\hline Tetrachloroethylene & ND & ND & & 0.2 & 0.2 & 0.0 & 0.4 & 0.4 & 0.0 & 0.0 \\
\hline Ethylbenzene & 0.2 & 0.1 & 47.1 & 5.4 & 5.4 & 0.0 & 6.0 & 5.6 & 4.9 & 13.7 \\
\hline p-Xylene $+\mathrm{m}$-Xylene & 0.5 & 0.3 & 35.4 & 12.7 & 12.6 & 0.6 & 13.7 & 13.3 & 2.1 & 10.2 \\
\hline Bromoform & ND & ND & $\mathrm{NC}$ & ND & ND & NC & ND & ND & $\mathrm{NC}$ & $\mathrm{NC}$ \\
\hline Styrene & ND & ND & $\mathrm{NC}$ & ND & ND & NC & 0.3 & 0.4 & 20.2 & 10.1 \\
\hline 0 -Xylene & 0.2 & 0.2 & 0.0 & 2.5 & 2.7 & 5.4 & 3.1 & 3.1 & 0.0 & 1.6 \\
\hline n-Nonane & ND & ND & $\mathrm{NC}$ & 0.6 & 0.6 & 0.0 & 0.8 & 0.8 & 0.0 & 0.0 \\
\hline a-Pinene + Benzaldehyde & 0.2 & 0.2 & 0.0 & 0.9 & 0.7 & 17.7 & 0.7 & 0.8 & 9.4 & 5.8 \\
\hline o-Chlorotoluene & ND & ND & NC & ND & ND & NC & ND & 0.2 & NC & $\mathrm{NC}$ \\
\hline n-Propylbenzene & ND & ND & $\mathrm{NC}$ & 0.1 & 0.1 & 0.0 & 0.2 & ND & NC & 0.0 \\
\hline m-Ethyltoluene & ND & ND & NC & 0.2 & 0.2 & 0.0 & 0.3 & 0.3 & 0.0 & 0.0 \\
\hline p-Ethyltoluene & ND & ND & NC & 0.3 & 0.3 & 0.0 & 0.4 & 0.4 & 0.0 & 0.0 \\
\hline
\end{tabular}

ND

(2)


Table 3-8, Continued

\begin{tabular}{|c|c|c|c|c|c|c|c|c|c|c|}
\hline \multirow{3}{*}{ Compound } & \multicolumn{9}{|c|}{ Date Sampled } & \multirow{3}{*}{$\begin{array}{l}\text { Pooled } \\
\text { cV } \\
(\%)\end{array}$} \\
\hline & \multicolumn{3}{|c|}{$7 / 28 / 94$} & \multicolumn{3}{|c|}{$7 / 29 / 94$} & \multicolumn{3}{|c|}{$8 / 01194$} & \\
\hline & A & B. & $\mathrm{CV}(\%)$ & A & B & $\mathrm{CV}(\%)$ & A & B. & $\mathrm{CV}(\%)$ & \\
\hline 1,3,5-Trimethylbenzene & ND & ND & $\mathrm{NC}$ & 0.2 & 0.2 & 0.0 & 0.4 & 0.4 & 0.0 & 0.0 \\
\hline o-Ethyltoluene & ND & ND & $\mathrm{NC}$ & 0.2 & 0.2 & 0.0 & ND & ND & NC & 0.0 \\
\hline b-Pinene & ND & ND & $\mathrm{NC}$ & ND & ND & $\mathrm{NC}$ & 0.1 & ND & NC & NC \\
\hline 1,2,4-TMBenzene $+t$-Butylbenzene & ND & ND & $\mathrm{NC}$ & 0.5 & 0.5 & 0.0 & 0.7 & 0.8 & 9.4 & 3.3 \\
\hline n-Decane $+p$-Dichlorobenzene & 0.2 & 0.1 & 47.1 & 0.9 & 0.8 & 8.3 & 1.0 & 1.1 & 6.7 & 14.0 \\
\hline 1,2,3-Trimethylbenzene & ND & ND & $\mathrm{NC}$ & 0.1 & 0.1 & 0.0 & 0.3 & 0.3 & 0.0 & 0.0 \\
\hline Limonene & ND & ND & $\mathrm{NC}$ & 0.2 & ND & $\mathrm{NC}$ & ND & 0.1 & $\mathrm{NC}$ & NC \\
\hline n-Butylbenzene & ND & ND & $\mathrm{NC}$ & 0.1 & 0.1 & 0.0 & 0.1 & 0.1 & 0.0 & 0.0 \\
\hline p-Diethylbenzene & ND & ND & $\mathrm{NC}$ & 0.09 & ND & $\mathrm{NC}$ & 0.2 & 0.2 & 0.0 & 0.0 \\
\hline n-Undecane & 0.3 & 0.3 & 0.0 & 0.4 & 0.5 & 15.7 & 0.7 & 0.8 & 9.4 & 5.3 \\
\hline Naphthalene & ND & ND & $\mathrm{NC}$ & ND & ND & $\mathrm{NC}$ & ND & ND & $\mathrm{NC}$ & NC \\
\hline Total Unidentified VOCs & 9.5 & 5.8 & 34.2 & 43.7 & 47.2 & 5.4 & 38.8 & 35.7 & 5.9 & 10.1 \\
\hline Total Unidentified Halogenated VOCs & ND & ND & NC & 1.9 & 1.5 & 16.6 & 0.5 & 0.8 & 32.6 & 13.0 \\
\hline TNMHC & 113 & 109 & 2.5 & 679 & 728 & 4.9 & 344 & 328 & 3.4 & 1.9 \\
\hline
\end{tabular}


TA-54 Dome Study

Table 3-9

Summary of Area L VOC Results

\begin{tabular}{|c|c|c|c|}
\hline Compound & Site ${ }^{1}$ & Site $2^{2}$ & Site $3^{3}$ \\
\hline Ethylene & 0.3 & 0.2 & 0.4 \\
\hline Ethane & 0.8 & 0.7 & 0.6 \\
\hline Chlorodifluoromethane & 0.1 & 0.1 & ND \\
\hline Propylene & 0.3 & 0.2 & 0.3 \\
\hline Propane & 9.4 & 1.1 & 0.5 \\
\hline Chloromethane & 0.4 & 0.3 & 0.3 \\
\hline Dichlorodifluoromethane & 2.2 & 0.4 & 0.4 \\
\hline Isobutane & 1.6 & ND & ND \\
\hline n-Butane & 3.4 & 0.3 & ND \\
\hline Methanol & 4.1 & 4.0 & 1.0 \\
\hline Isopentane & 0.3 & 0.4 & 0.3 \\
\hline Acetone & 1.4 & 8.5 & 2.5 \\
\hline Trichlorofluoromethane & 0.6 & 0.3 & 0.7 \\
\hline n-Pentane & 0.2 & ND & ND \\
\hline Isoprene & ND & 0.2 & ND \\
\hline 1,1-Dichloroethylene & 0.4 & ND & ND \\
\hline Methylene Chloride & 17.6 & 5.4 & 7.7 \\
\hline Freon 113 & 0.9 & 0.2 & ND \\
\hline 1,1-Dichloroethane & ND & ND & 0.2 \\
\hline 2-Butanone & 1.2 & ND & ND \\
\hline n-Hexane & 0.7 & 0.5 & ND \\
\hline Chloroform & 0.1 & 0.2 & 0.04 \\
\hline 1,1,1-Trichloroethane & 2.3 & 2.2 & 3.5 \\
\hline Benzene & 0.1 & ND & ND \\
\hline Carbon Tetrachloride & 0.1 & 0.1 & 0.1 \\
\hline 1-Butanol + Cyclohexane & ND & 0.7 & ND \\
\hline Trichloroethylene + BDCMethane & 0.2 & 0.2 & 0.2 \\
\hline Toluene & 1.0 & 0.7 & 0.2 \\
\hline Tetrachloroethylene & 0.1 & 0.1 & ND \\
\hline $\mathrm{p}$-Xylene + $\mathrm{m}$-Xylene & ND & 0.1 & ND \\
\hline Total Unidentified VOCs & 1.8 & 2.1 & 3.6 \\
\hline Unidentified Halogenated VOCs & ND & ND & ND \\
\hline TNMHC & 15.0 & 10.8 & 6.3 \\
\hline
\end{tabular}

${ }^{1}$ Site 1 located between rows 39 and 41 in Area $L$ mixed waste storage pad.

${ }^{2}$ Site 2 located between rows 63 and 65 in Area $L$ mixed waste storage pad.

${ }^{3}$ Site 3 located at the southeast end of the mixed waste storage pad. 


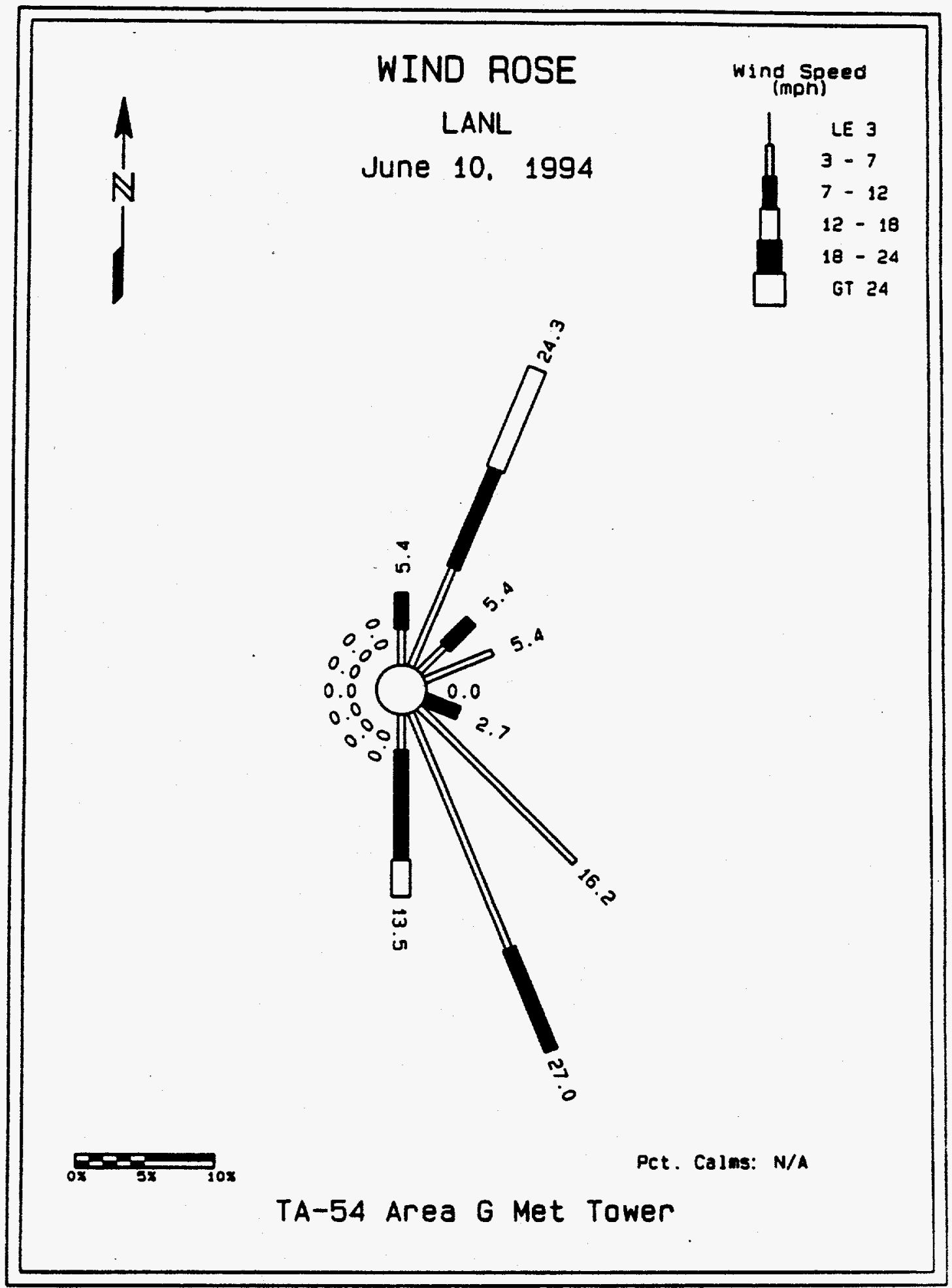

Figure 3-1. Wind Rose for June 10, 1994. 


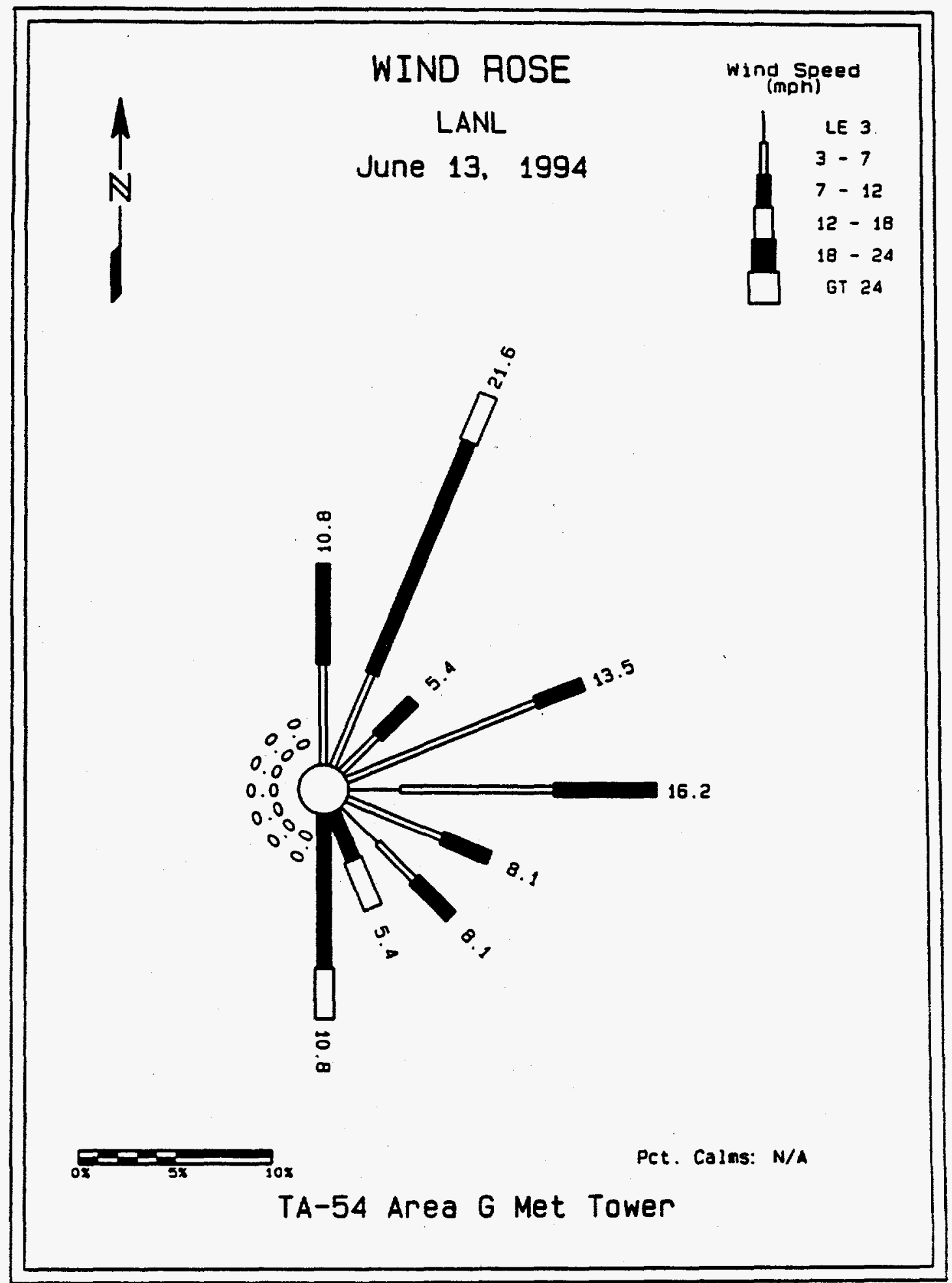

Figure 3-2. Wind Rose for June 13, 1994. 
TA-54 Dome Study

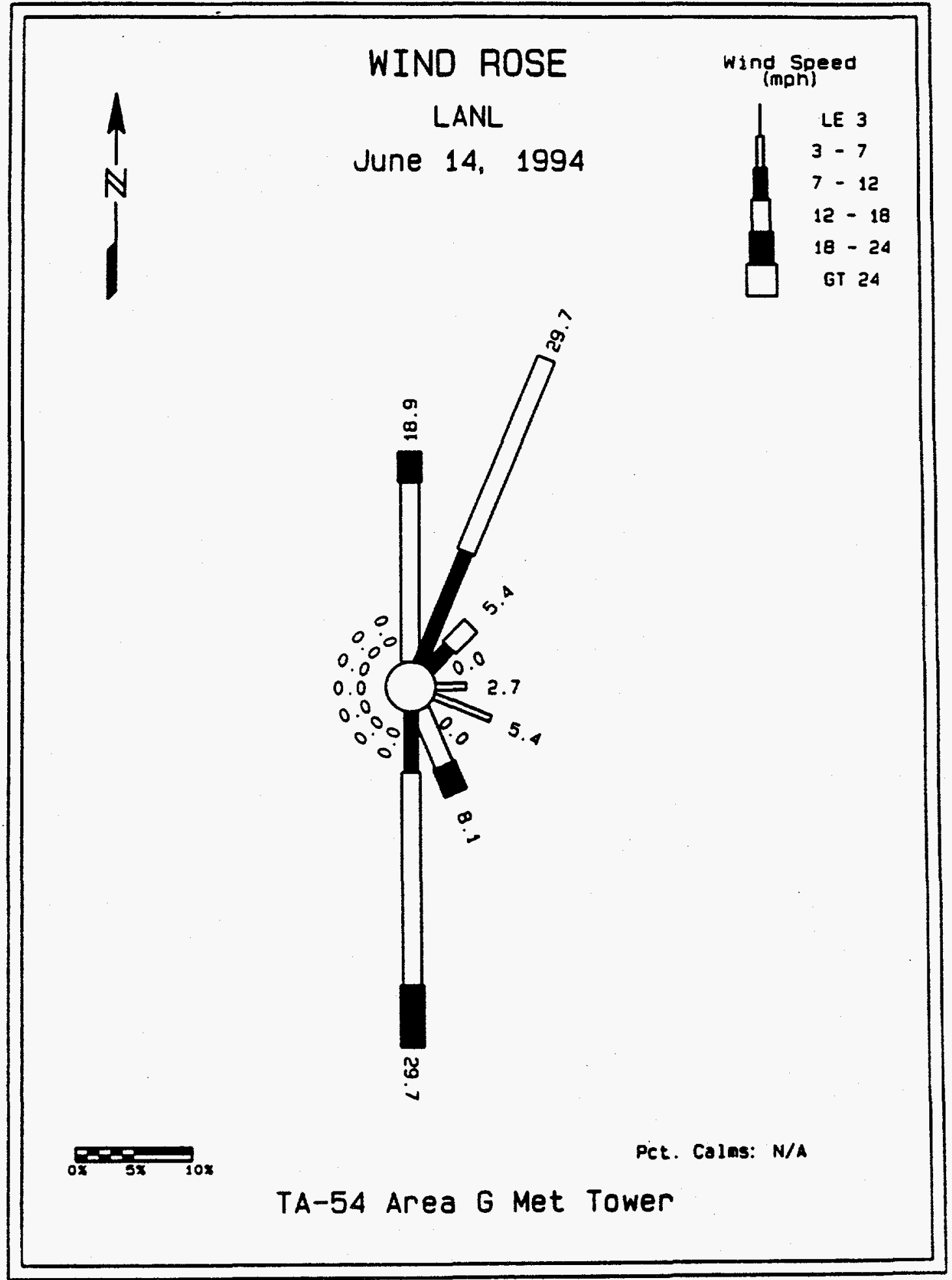

Figure 3-3. Wind Rose for June 14, 1994. 


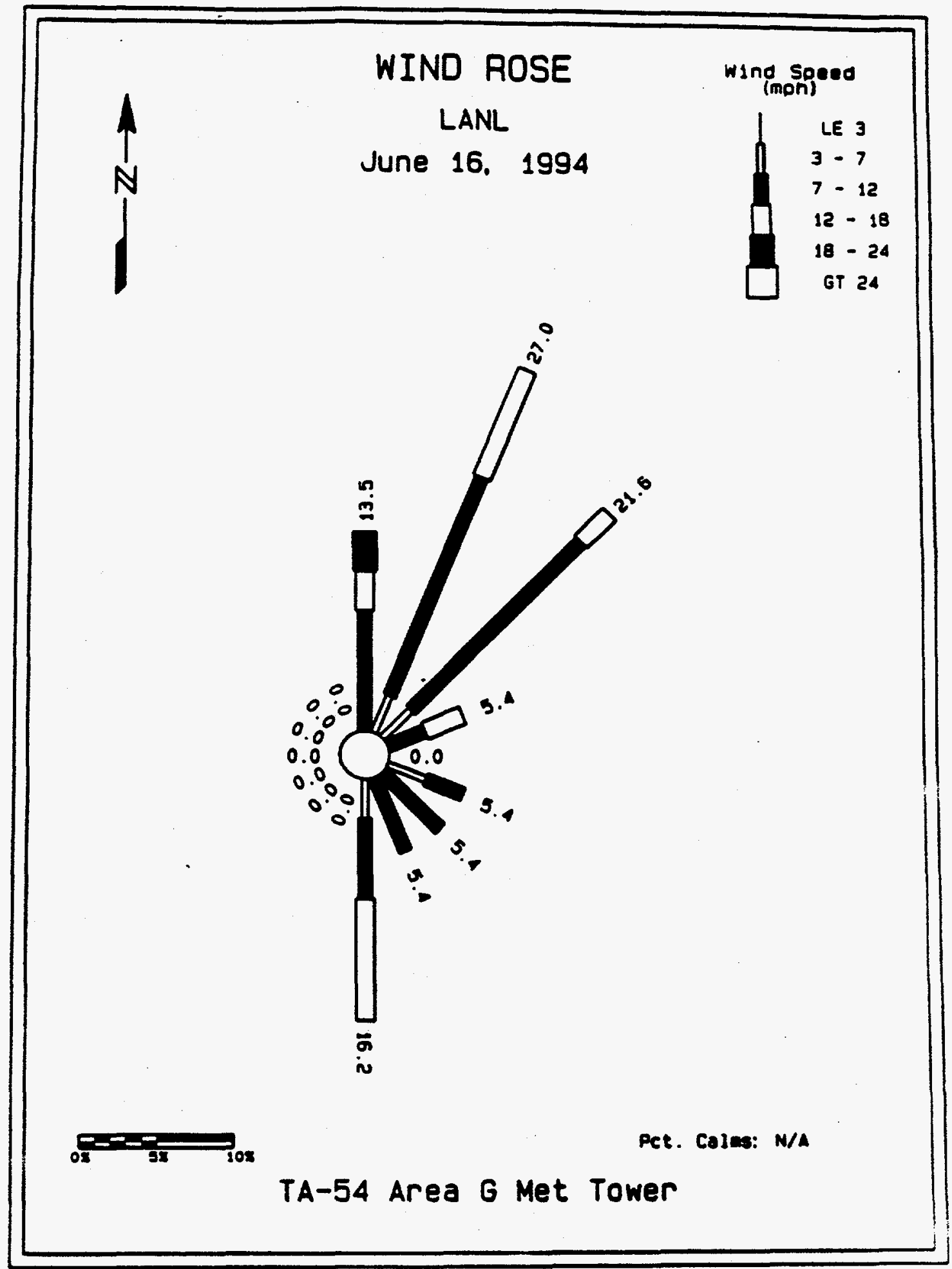

Figure 3-4. Wind Rose for June 16, 1994. 
TA-54 Dome Study

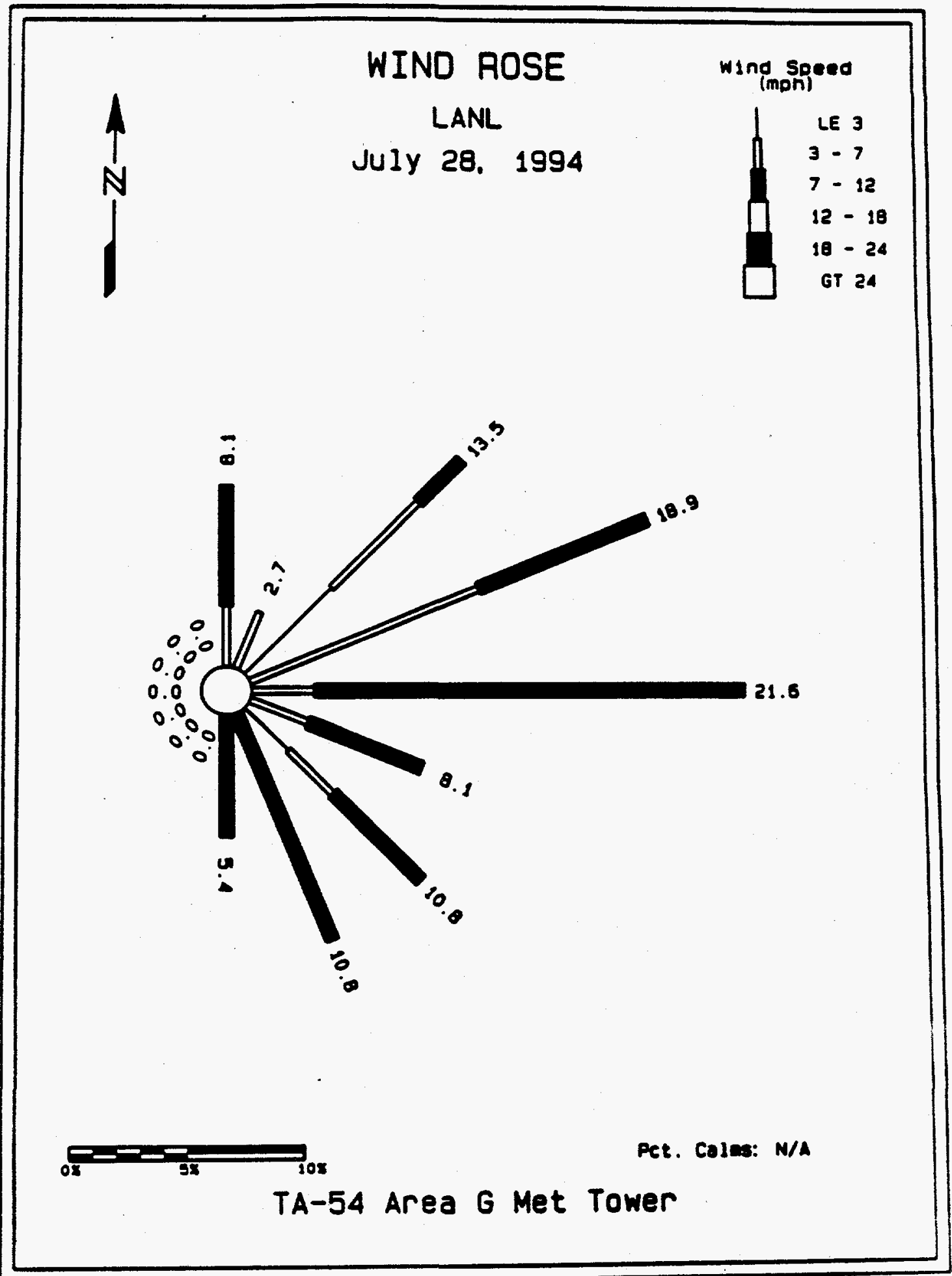

Figure 3-5. Wind Rose for July 28, 1994. 


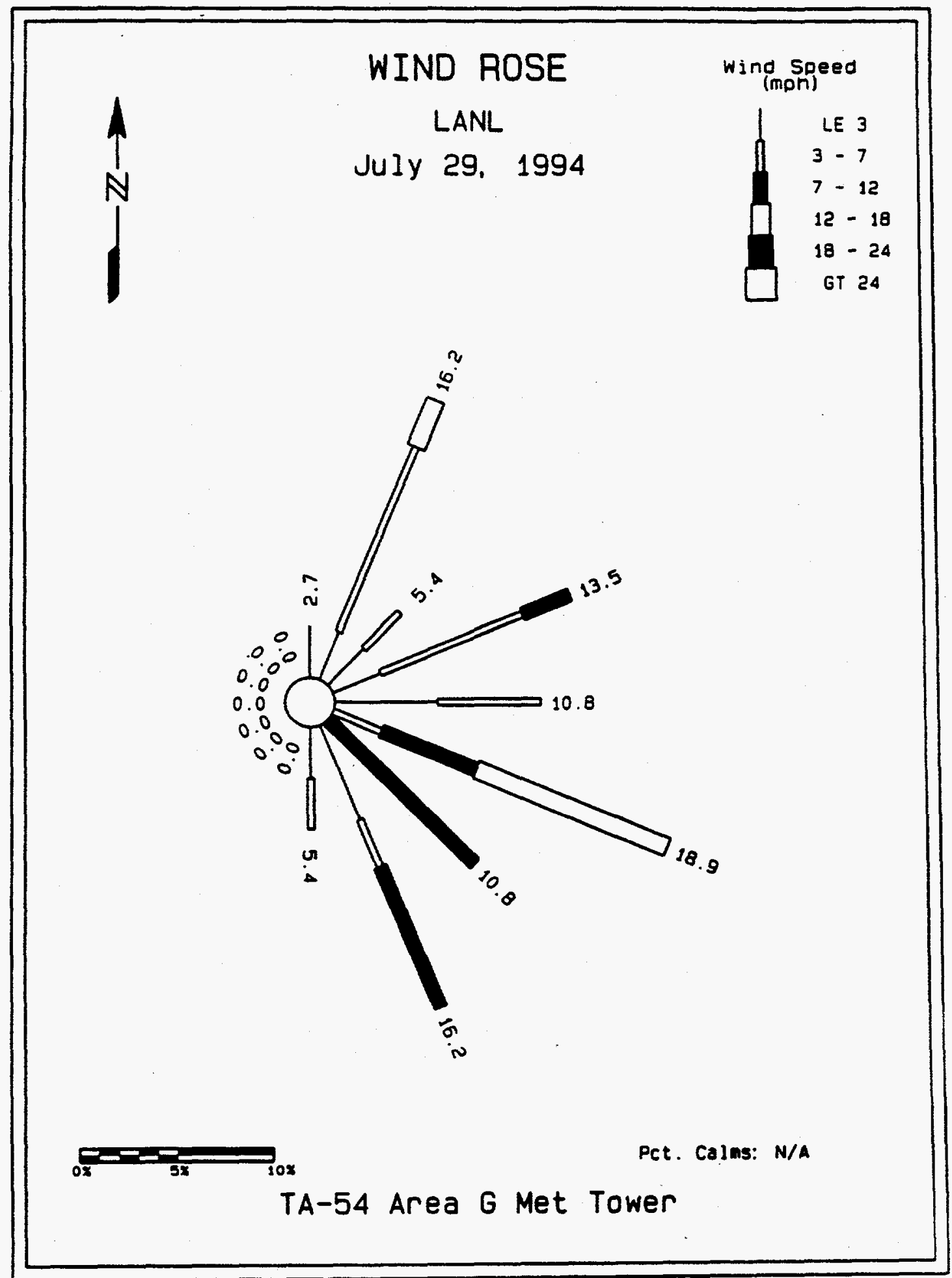

Figure 3-6. Wind Rose for July 29, 1994. 


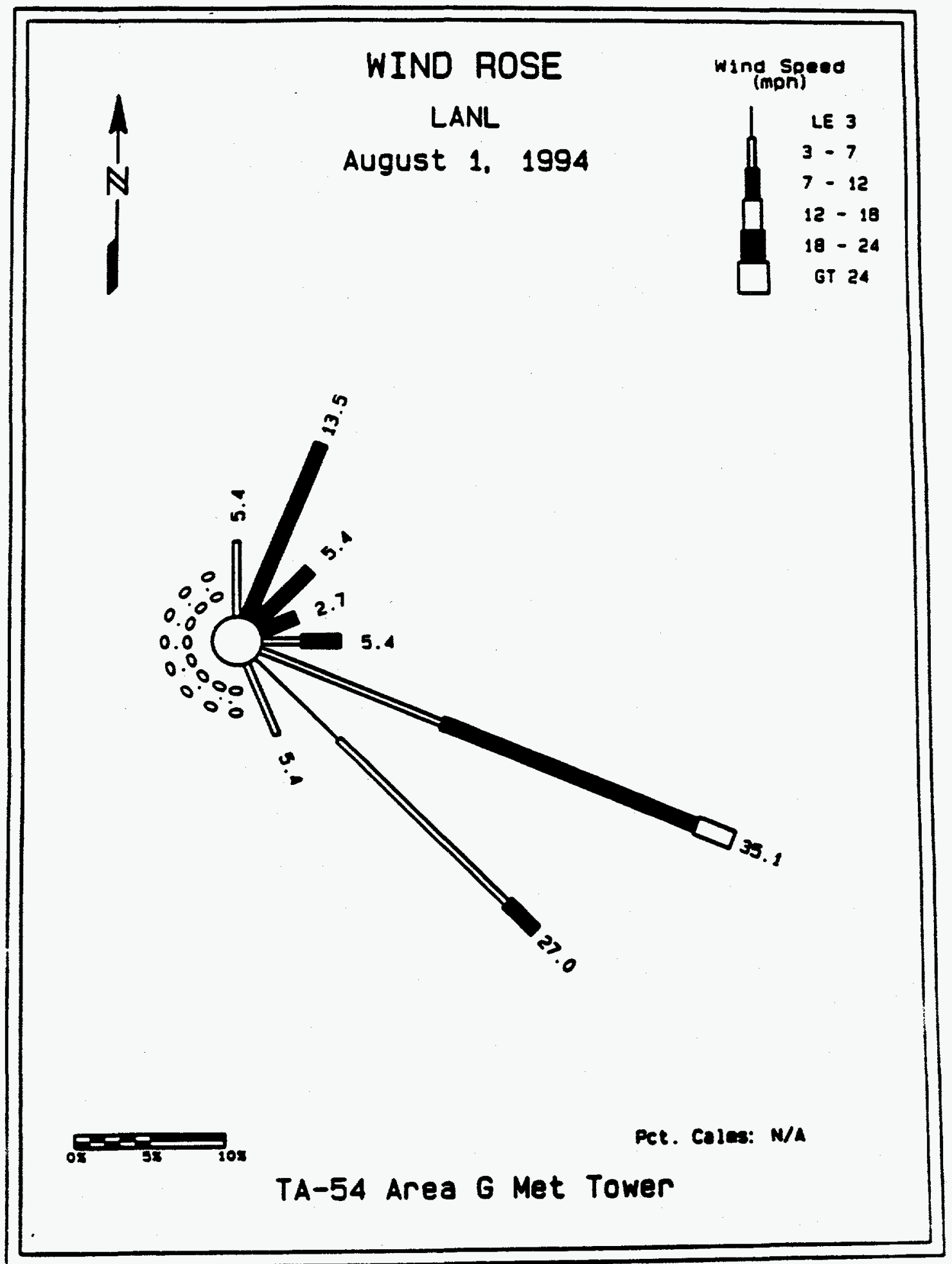

Figure 3-7. Wind Rose for August 1, 1994. 



\subsection{DISCUSSION OF RESULTS}

This section discusses the VOC and tritium monitoring results. Spatial variability within the domes, variability among the domes, the effects on operating parameters, correlations with meteorological conditions, a comparison of measured values with applicable health standards, and estimated annual analyte emissions are discussed. Monitoring results were presented in Section 3.

\subsection{Spatial Variability Within Domes}

It was expected that, the sample variability would be large given the nature of the storage domes (i.e., they are very long, are not well-mixed, and house a variety of wastes). In the original proposal, Radian recommended that a total of five points within each dome be monitored to help minimize the potential for bias due to compound stratification within the domes. The analytical results showed the opposite to be the case, however. In general, spatial variability was fairly small, indicating that more mixing within the domes was occurring than expected. Table 4-1 shows variability estimates for the tritium monitoring while Table 4-2 shows the variability estimates for selected VOCs. Variability estimates are only shown for domes where three sampling points were monitored (i.e., Domes 49 and 153).

Generally, the variability between sampling points, within each dome, were similar to the sampling variability alone (Table 3-4 for tritium and Table 3-8 for VOCs). The tritium variability, as measured by percent coefficient of variation (\%CV), ranged from $7.9 \%$ to $89.6 \%$ with a pooled CV of $15.6 \%$ for Dome 153 (six sampling days) and $30.8 \%$ (three sampling days) for Dome 49. The majority of the sampling days had CV's less than these pooled values, with the pooled values being biased by two possible outlier points. These data tend to indicate that either the wastes stored in the domes are fairly homogenous or that significant air mixing is occurring inside the domes, even when they are closed. While wastes inside the domes are organized by their waste characteristics, the chances are minimal that the emission rates for these individual wastes are constant along the entire length of the domes.

Table 4-2 shows CVs for selected compounds from Domes 49 and 153. The variability between sampling points for the VOCs were similar to what was observed for the tritium samples. The lone exception was methylene chloride in Dome 49, which had striking differences between points. The CVs for most sampling points were less than $30 \%$, which was the analytical precision objective for the project. This mirrored the tritium results and again suggests that the domes are generally better mixed than expected.

The methylene chloride values measured in Dome 49, had a pooled CV of $69 \%$ for the three sampling periods. Since the relative magnitude of the differences between points were consistent for each of the three sampling periods, this indicates that sample point B (see Figures 2-1 through 2-3 for sample locations) may have been located near a localized hot-spot for methylene chloride. It was interesting that other measured hydrocarbons in this 


\section{Table 4-1 \\ Summary of Tritium Spatial Variability Estimates}

\begin{tabular}{|c|c|c|c|c|c|}
\hline \multirow{2}{*}{ Sample Date } & \multicolumn{3}{|c|}{ Sample Point $\left(\mathrm{pCi} / \mathrm{m}^{3}\right)$} & \multirow{2}{*}{ Concentration } & \multirow{2}{*}{ CV (\%) } \\
\hline & Sample A & Sample B & Sample C & & \\
\hline \multicolumn{6}{|c|}{ Dome 153} \\
\hline $6 / 10 / 94$ & 60,000 & 58,600 & 69,100 & 62,600 & 9.1 \\
\hline $6 / 13 / 94$ & 55,900 & 45,200 & 58,800 & 53,300 & 13.4 \\
\hline $6 / 14 / 94$ & 2,780 & 3,060 & 7,340 & 4,390 & 58.2 \\
\hline $7 / 28 / 94$ & 4,250 & 4,430 & 5,930 & 4,780 & 18.9 \\
\hline $7 / 29 / 94$ & 54,200 & 40,700 & 56,900 & 50,600 & 17.2 \\
\hline $8 / 01 / 94$ & 64,600 & 59,500 & 69,900 & 64,700 & 8.0 \\
\hline \multicolumn{6}{|c|}{ Dome 49} \\
\hline $7 / 28 / 94$ & 168 & 108 & 135 & 137 & 21.9 \\
\hline $7 / 29 / 94$ & 343 & 1,372 & 308 & 674 & 89.6 \\
\hline $8 / 01 / 94$ & 497 & 575 & 511 & 528 & 7.9 \\
\hline
\end{tabular}

Note: Samples collected on 6/14/94 and 7/28/94 were collected with domes opened. Samples collected on 6/10/94 and 7/29/94 were collected after domes had been closed overnight and samples collected on 6/13/94 and 8/01/94 were collected after domes had been closed over a weekend. 
Table 4-2

Summary of VOC Spatial Variability Estimates

\begin{tabular}{|c|c|c|c|c|c|}
\hline \multirow[b]{2}{*}{ Sample Date } & \multicolumn{3}{|c|}{ Sample Point (ppbv) } & \multirow{2}{*}{ Concentration } & \multirow[b]{2}{*}{ CV (\%) } \\
\hline & Sample A & Sample B & Sample C & & \\
\hline \multicolumn{6}{|c|}{ Dome 153 - Bromomethane } \\
\hline $6 / 10 / 94$ & 40.3 & 43.0 & 56.5 & $\overline{46.6}$ & 18.6 \\
\hline $6 / 13 / 94$ & 47.0 & 39.8 & 53.6 & 46.8 & 14.7 \\
\hline $6 / 14 / 94$ & 2.8 & ND & 4.6 & 3.7 & $\mathrm{NC}$ \\
\hline $7 / 28 / 94$ & ND & ND & ND & ND & $\mathrm{NC}$ \\
\hline $7 / 29 / 94$ & 17.4 & 23.7 & 40.6 & $\overline{27.2}$ & 44.1 \\
\hline $8 / 01 / 94$ & 43.4 & 30.6 & 46.6 & 40.2 & 21.1 \\
\hline \multicolumn{6}{|c|}{ Dome 153 - 1,1,1-Trichloroethane } \\
\hline $6 / 10 / 94$ & 61.8 & 75.4 & 72.2 & $\overline{69.8}$ & 10.2 \\
\hline $6 / 13 / 94$ & 54.7 & 60.0 & 66.5 & $\overline{60.4}$ & 9.8 \\
\hline $6 / 14 / 94$ & 5.6 & 5.5 & 11.2 & $\overline{7.4}$ & 43.9 \\
\hline $7 / 28 / 94$ & 1.6 & 2.5 & 2.5 & 2.2 & 23.6 \\
\hline $7 / 29 / 94$ & 19.4 & 29.1 & 19.0 & 22.5 & 25.4 \\
\hline $8 / 01 / 94$ & 19.2 & 25.6 & 21.6 & 22.1 & 14.6 \\
\hline \multicolumn{6}{|c|}{ Dome 153 - TNMHC } \\
\hline $6 / 10 / 94$ & 30.2 & 32.2 & 46.3 & $\overline{36.2}$ & 24.2 \\
\hline $6 / 13 / 94$ & 44.6 & 35.1 & 34.3 & 38.0 & 15.1 \\
\hline $6 / 14 / 94$ & $\overline{5.5}$ & 12.6 & 23.0 & 13.7 & 64.2 \\
\hline $7 / 28 / 94$ & 6.4 & 9.6 & 8.0 & 8.0 & 20.0 \\
\hline $7 / 29 / 94$ & 23.4 & 38.1 & 29.2 & 30.2 & 24.5 \\
\hline $8 / 01 / 94$ & 32.6 & 81.4 & 30.3 & 48.1 & 60.0 \\
\hline \multicolumn{6}{|c|}{ Dome 49 - Propane } \\
\hline $7 / 28 / 94$ & 160 & 189 & 261 & 203 & 25.6 \\
\hline $7 / 29 / 94$ & 899 & 958 & 850 & 902 & 6.0 \\
\hline $8 / 01 / 94$ & 307 & 303 & 241 & 284 & 13.0 \\
\hline \multicolumn{6}{|c|}{ Dome 49 - Methylene Chloride } \\
\hline $7 / 28 / 94$ & 13.6 & 126 & 72.5 & 70.7 & 79.5 \\
\hline $7 / 29 / 94$ & 473 & 1,280 & 548 & 767 & 58.1 \\
\hline $8 / 01 / 94$ & 480 & 1,450 & 505 & 812 & 68.1 \\
\hline
\end{tabular}


Table 4-2

(Continued)

\begin{tabular}{|c|c|c|c|c|c|}
\hline \multirow[b]{2}{*}{ Sample Date } & \multicolumn{3}{|c|}{ Sample Point (ppbv) } & \multirow{2}{*}{ Concentration } & \multirow[b]{2}{*}{ cV (\%) } \\
\hline & Sample A & Sample B & Sample C & & \\
\hline \multicolumn{6}{|c|}{ Dome 49 - Toluene } \\
\hline $7 / 28 / 94$ & 1.1 & 1.7 & 2.3 & 1.7 & 35.3 \\
\hline $7 / 29 / 94$ & 15.5 & 21.2 & 19.1 & 18.6 & 15.5 \\
\hline $8 / 01 / 94$ & 18.1 & 22.8 & 22.1 & 21.0 & 12.1 \\
\hline \multicolumn{6}{|c|}{ Dome 49 - TNMHC } \\
\hline $7 / 28 / 94$ & 87.9 & 113 & 146 & 116 & 25.2 \\
\hline $7 / 29 / 94$ & $\overline{574}$ & 679 & 584 & 612 & $\overline{9.5}$ \\
\hline $8 / 01 / 94$ & 275 & 344 & 279 & 299 & 12.9 \\
\hline
\end{tabular}

Note: Samples collected on 6/14/94 and 7/28/94 were collected with domes opened. Samples collected on 6/10/94 and 7/29/94 were collected after domes had been closed overnight and samples collected on 6/13/94 and 8/01/94 were collected after domes had been closed over a weekend. 
dome did not display the same degree of stratification. Collocated VOC samples collected at this point showed good agreement, further confirming the methylene chloride values.

\subsection{Variability of Analyte Concentrations Among Domes}

Each of the three domes in Area G are used to store different materials (i.e., FRP in Dome 153, Certified TRU in Dome 48, and mixed waste in Dome 49). Therefore, significant differences in analyte composition and concentration are expected. Dome 49, for instance, had relatively high levels of VOCs and relatively low levels of tritium while Dome 153 (FRP dome) was the exact opposite.

For example, the average tritium concentration in Dome 49 as $137 \mathrm{pCi} / \mathrm{m}^{3}$ when open and was $600 \mathrm{pCi} / \mathrm{m}^{3}$ when closed. In contrast, the average tritium concentration in Dome 153 was 4,630 $\mathrm{pCi} / \mathrm{m}^{3}$ when open and $57,800 \mathrm{pCi} / \mathrm{m}^{3}$ when closed. Dome 48 had tritium concentrations lower than Dome 153 but still higher than 49 . When open, average tritium concentrations were $1,720 \mathrm{pCi} / \mathrm{m}^{3}$ and were $11,600 \mathrm{pCi} / \mathrm{m}^{3}$ when closed. This dome appears to have had some stratification of tritium concentrations shown by point $\mathrm{A}$ being approximately $25 \%$ lower than point B when the dome was closed and being $70 \%$ or more lower when the dome was open. This was not observed in the other two domes. Since only two points were monitored inside this dome, it isn't possible to make statements about which point is most representative of the overall dome concentration.
The VOC concentrations varied considerably in both concentration and composition between domes. While halogenated compounds seemed to predominate, the predominate specific compound tended to be different for each of the three domes. Dome 153 had the lowest overall VOC concentrations, followed by dome 48 , with Dome 49 having the highest VOC concentrations.

Dome 153 had values for total nonmethane hydrocarbon (TMNHC) concentrations, based on a carbon number of 6, ranging from a high of $81 \mathrm{ppb}$ to a low of $6 \mathrm{ppb}$ and a mean value of $48 \mathrm{ppb}$. As a comparison, the ambient sample concentrations collected at site 2 (located approximately 150 feet northwest of the dome) ranged from $3.3 \mathrm{ppb}$ to $27 \mathrm{ppb}$ and averaged $9.2 \mathrm{ppb}$. The predominate species found in Dome 153 were bromomethane, methylene chloride, and freon 113. Methylene chloride and freon 113 were detected in the other two domes, however, bromomethane was only detected in this dome.

Methanol was also detected at elevated concentrations in several canisters, however, the quantitation certainty of this compound is in doubt. Methanol was used by the manufacturer to clean the canister parts. When the canisters were received, many very rigorous cleanings were required to remove the methanol from the canisters. Even after these cleanings, methanol continued to be a problem in some canisters at low levels. Therefore, methanol values should be considered highly suspect. 
Major species in Dome 48 included 1,1-dichloroethane, 1,1,1-trichloroethane, toluene, acetone, and chloromethane with 1,1,1-trichloroethane being the predominate species. TMNHC (based on a carbon number of 6) values averaged 12 $\mathrm{ppb}$ when the dome was open and $90 \mathrm{ppb}$ when the dome was closed. Methanol was also detected in several of the canisters collected inside Dome 48. As discussed earlier, these values may be suspect. 1,1,1-trichloroethane values averaged 430 ppb ( $\pm 74 \mathrm{ppb})$ when the domes were closed and averaged $87 \mathrm{ppb}( \pm 50 \mathrm{ppb})$ when open. When the dome was closed, the compound variability between the two sampling points were less than when open.

As expected, Dome 49 had the highest VOC concentrations of the three domes. Major species included propane, methylene chloride, 1,1,1,-trichloroethane, toluene, trichloroethylene and bromodichloromethane (coeluters), and diethyl ether and 2-propanol (coeluters). While the chlorinated compounds were of no surprise, the high levels of propane and other light alkanes and alkenes were unexpected. These values may be due to fugitive emissions from a propane powered forklift used for moving pallets inside the dome. TMNHC values for this dome ranged from and average of $116 \mathrm{ppb}$ when open to $612 \mathrm{ppb}$ when closed overnight and $299 \mathrm{ppb}$ when closed over the weekend. The main differences between these values seems to be the propane values which averaged over $900 \mathrm{ppb}$ on July 28 th and averaged approximately 280 $\mathrm{ppb}$ on August 1st. Most of the chlorinated compounds had very similar concentrations between the two sampling periods when the domes were closed, which indicates that the source of the propane and other light alkanes may have been different from the chlorinated solvents.

\subsection{Effects of Operating Practices on Air Quality}

This study evaluated the effect of operating practices on VOC and tritium concentrations. Samples were collected while the domes were open, after they had been closed overnight, and after they had been closed for a weekend. Table 4-3 shows average analyte concentration (selected VOCs and tritium) under the three operational conditions. As expected, the concentrations when the domes were open were significantly lower than when they were closed. The differences between when the domes had been closed overnight and over the weekend were less pronounced. There were usually less than $10 \%$ differences between the two conditions and for some analytes, the values were the same or even slightly lower. This was not expected since none of the values measured even approached saturation values which could have caused backpressure and consequently repressed vapor values. It appears that both VOC and tritium concentrations reach maximum concentrations quickly. This is shown by the relatively small differences in the analyte concentrations measured after the domes had been closed for 16 hours and after 64 hours.

The reasons for this are not apparent. It is possible that there is more 
Table 4-3

Comparison of Analyte Concentration Based on Dome Condition

\begin{tabular}{|c|c|c|c|}
\hline \multirow{2}{*}{ Compound't } & \multicolumn{3}{|c|}{ Dome Condition } \\
\hline & Open ${ }^{2}$ & Overnight Closure'? & Weekend Closure' \\
\hline \multicolumn{4}{|c|}{ Dome 48 (Concentrations) } \\
\hline Tritium & 1,720 & 10,300 & 12,800 \\
\hline 1,1-Dichloroethane & 3.1 & 11.4 & 15.1 \\
\hline 1,1,1-Trichloroethane & 87 & 368 & 493 \\
\hline Toluene & 1.1 & 7.1 & 6.1 \\
\hline Chloromethane & 1.2 & 3.8 & 5.2 \\
\hline \multicolumn{4}{|c|}{ Dome 49 (Concentrations) } \\
\hline Tritium & 137 & 674 & 528 \\
\hline Propane & 203 & 902 & 284 \\
\hline 1,1,1-Trichloroethane & 0.6 & 5.9 & 7.5 \\
\hline Toluene & 1.7 & 18.6 & 21.0 \\
\hline Methylene Chloride & 71 & 767 & 812 \\
\hline \multicolumn{4}{|c|}{ Dome 153 (Concentrations) } \\
\hline Tritium & 4,630 & 56,600 & 59,000 \\
\hline Bromomethane & 1.2 & 36.9 & 43.5 \\
\hline Methylene Chloride & 0.2 & 2.8 & 2.8 \\
\hline Freon 113 & 0.6 & 10.2 & 8.4 \\
\hline 1,1,1-Trichloroethane & 4.8 & 46.2 & 41.2 \\
\hline
\end{tabular}

${ }^{1} \mathrm{VOC}$ concentrations in units of ppbv.

Tritium concentrations in units of $\mathrm{pCi} / \mathrm{m}^{3}$.

${ }^{2}$ Samples collected while domes were open.

${ }^{3}$ Samples collected after domes had been closed for 16 hours.

${ }^{4}$ Samples collected after domes had been closed for 64 hours. 
through other pathways. The other unknown is how the constituents are distributed vertically within the domes. While the data seem to indicate that the analytes are generally fairly evenly dispersed within the horizontal plane, in the breathing zone, the vertical distribution can not be assessed from the existing data. Again, it is possible that the concentrations near the top of the domes are higher than in the breathing zone. Additional data would be required to verify either of these postulations.

\subsection{Comparison of Measured Values with Applicable Health Standards}

Tables 4-4 (domes closed) and 4-5 (domes open) compare selected VOCs and tritium with occupational exposure values. The table shows the minimum, maximum, and average concentrations within each dome for selected VOCs. The occupational values (VOCs only) presented in the table are Threshold Limit Values (TLVs) - Time Weighted Averages (TWAs) published by the American Conference of Governmental Industrial Hygienists (ACGIH). These values represent airborne concentrations of substances that are thought to not pose health risks to nearly all workers who may be exposed to these agents repeatedly, day after day (i.e., 8 hours per day, 5 days per week, 50 weeks per year). These concentrations are not designed to protect the most sensitive individuals, such as young children or the elderly, but healthy adult workers.

ACGIH does not have a TLV for tritium. The Nuclear Regulatory Commission (NRC) has, however, established Derived Air Concentrations (DACs) for tritium $(2.0 \mathrm{E}-05 \mu \mathrm{Ci} / \mathrm{ml}$ or $2.0 \mathrm{E}+07 \mathrm{pCi} / \mathrm{m}^{3}$ ). This concentration value represents the maximum Annual Limit Intake (ALI) for an individual, who does not receive any other radionuclide exposure. This is an annual value, therefore, if an individual were to only be exposed for half the year, this value could be doubled. Conversely, if there were other exposures, this value would need to be reduced accordingly. DAC values are published in 10 CFR20, Appendix B. Since the objective of the monitoring was to assess worker exposure, DAC's are appropriate for worker exposure assessments.

The data show that even with the domes closed, VOC concentrations did not approach concentrations of concern from a worker exposure standpoint. The highest concentrations inside any of the domes were for methylene chloride, which had values approaching $1.5 \mathrm{ppm}$. The TWA for methylene chloride is $50 \mathrm{ppm}$, making the highest exposure less than three percent of the allowable TWA. All other maximum VOC concentrations were several orders of magnitude lower than allowable TWAs. For tritium, the highest measured value was $69,900 \mathrm{pCi} / \mathrm{m}^{3}$. This is less than one percent of the DAC of $20,000,000 \mathrm{pCi} / \mathrm{m}^{3}$, even considering other potential exposures. When the domes were open, these values are even less significant. Figure 4-1 shows a graph of selected VOCs and percent of TWA or DAC. 
Table 4-4

Comparison of Measured Analyte Values with ACGIH TWAs - Domes Closed

\begin{tabular}{|c|c|c|c|c|c|c|c|c|c|c|}
\hline \multirow{2}{*}{ Compound } & \multicolumn{3}{|c|}{ Dome 48} & \multicolumn{3}{|c|}{ Dome 49} & \multicolumn{3}{|c|}{ Done 153} & \multirow{2}{*}{$\begin{array}{l}\text { TWA } \\
(\mathrm{ppb})\end{array}$} \\
\hline & Min & Max & Mean & Min & Max & Mean & Min & $\operatorname{Max}$ & Mean & \\
\hline Tritium $^{2}$ & 7,620 & 15,900 & 11,600 & 308 & 1,372 & 601 & 40,700 & 69,900 & 57,800 & $2.0 \mathrm{E}+07^{2}$ \\
\hline Propane & 2.0 & 11.2 & 4.9 & 241 & 958 & 593 & 2.0 & 3.7 & 2.9 & $\mathrm{NA}^{3}$ \\
\hline Chloromethane & 3.1 & 7.0 & 4.5 & 0.3 & 1.0 & 0.6 & 1.8 & 4.0 & 2.4 & $\mathrm{NA}^{4}$ \\
\hline Bromomethane & ND & ND & ND & ND & ND & ND & 17.4 & 56.5 & 40.2 & $\mathbf{N A}^{4}$ \\
\hline Acetone & 17.1 & 31.3 & 21.2 & 13.5 & 47.9 & 27.5 & 2.8 & 54.4 & 8.8 & 750,000 \\
\hline 1,1-Dichloroethylene & 3.4 & 6.1 & 4.5 & ND & ND & ND & ND & 0.9 & 0.2 & 5,000 \\
\hline Methylene Chloride & 1.4 & 2.1 & 1.8 & 473 & 1,450 & 789 & 2.1 & 3.5 & 2.8 & 50,000 \\
\hline Freon 113 & 1.9 & 2.4 & 2.1 & 15.0 & 57.5 & 30.3 & 7.1 & 13.2 & 9.3 & $1,000,000$ \\
\hline 1,1-Dichloroethane & 9.5 & 15.9 & 13.3 & 0.2 & 0.4 & 0.3 & 0.1 & 0.4 & 0.2 & 100,000 \\
\hline 1,1,1-Trichloroethane & 330 & 529 & 430 & 4.6 & 8.2 & 6.7 & 19.0 & 75.4 & 43.7 & 350,000 \\
\hline Benzene & 0.6 & 1.5 & 0.8 & 0.7 & 1.3 & 1.0 & 0.3 & 0.6 & 0.5 & 10,000 \\
\hline Toluene & 4.7 & 10.5 & 6.6 & 15.5 & 22.8 & 19.8 & 2.0 & 3.5 & 2.7 & 50,000 \\
\hline Tetrachloroethylene & 1.2 & 2.9 & 2.3 & 0.2 & 0.4 & 0.3 & 0.2 & 0.6 & 0.4 & 1,000 \\
\hline p- \& m-Xylene & 1.8 & 3.1 & 2.5 & 4.5 & 13.7 & 8.5 & 0.4 & 0.8 & 0.6 & 100,000 \\
\hline o-Xylene & 0.6 & 1.0 & 0.8 & 1.0 & 5.0 & 2.5 & 0.2 & 0.4 & 0.3 & 100,000 \\
\hline
\end{tabular}

NOTE: All VOC concentrations in units of ppbv.

ND - Not Detected

' VOC TWA's in units of ppb, NOC tritium DAC in units of $\mu \mathrm{Ci} / \mathrm{ml}$. Normal convention calls for TWA's to be reported in units of ppm. These values

have been converted to allow easy comparision to field data.

${ }^{2}$ Tritium Concentrations in units of $\mathrm{pCi} / \mathrm{m}^{3}\left(\mathrm{pCi} / \mathrm{m}^{3} / 10^{12}=\mu \mathrm{Ci} / \mathrm{ml}\right)$

${ }^{3}$ Simple asphixiant, no TWA established.

${ }^{4}$ No TWA established. 
Table 4-5

Comparison of Measured Analyte Values with ACGIH TWAs - Domes Open

\begin{tabular}{|c|c|c|c|c|c|c|c|c|c|c|}
\hline \multirow{2}{*}{ Compound } & \multicolumn{3}{|c|}{ Dome 48} & \multicolumn{3}{|c|}{ Done 49} & \multicolumn{3}{|c|}{ Bone 153} & \multirow{2}{*}{$\begin{array}{l}\text { TWA } \\
(\mathrm{ppb})\end{array}$} \\
\hline & Min & Max & Mean. & Min. & Max. & Mean & Min. & Max: & Vean & \\
\hline Tritium $^{2}$ & 17.1 & 3,170 & 1,720 & 108 & 168 & 137 & 2,780 & 7,340 & 4,630 & $2.0 \mathrm{E}+0 T^{2}$ \\
\hline Propane & ND & 0.7 & 0.5 & 160 & 261 & 203 & 0.3 & 0.9 & 0.6 & $\mathbf{N A}^{3}$ \\
\hline Chloromethane & 0.1 & 3.0 & 1.2 & 0.3 & 0.4 & 0.4 & ND & 1.7 & 0.7 & $\mathbf{N A}^{4}$ \\
\hline Bromomethane & ND & ND & ND & ND & ND & ND & ND & 4.6 & 1.2 & $\mathrm{NA}^{4}$ \\
\hline Acetone & 0.4 & 2.8 & 1.5 & 3.1 & 5.9 & 4.2 & 1.4 & 2.9 & 2.0 & 750,000 \\
\hline 1,1-Dichloroethylene & 0.5 & 1.6 & 1.0 & ND & ND & ND & ND & ND & ND & 5,000 \\
\hline Methylene Chloride & 0.1 & 0.2 & 0.1 & 13.6 & 126 & 70.7 & 0.06 & 0.8 & 0.2 & 50,000 \\
\hline Freon 113 & 0.2 & 0.8 & 0.5 & ND & 1.0 & 0.3 & 0.1 & 1.1 & 0.6 & $1,000,000$ \\
\hline 1,1-Dichloroethane & 0.8 & 5.6 & 3.1 & ND & ND & ND & ND & ND & ND & 100,000 \\
\hline 1,1,1-Trichloroethane & 36.8 & 149 & 87.2 & 0.4 & 0.8 & 0.6 & 1.6 & 11.2 & 4.8 & 350,000 \\
\hline Benzene & 0.2 & 0.3 & 0.3 & 0.2 & 0.3 & 0.2 & 0.2 & 0.3 & 0.25 & 10,000 \\
\hline Toluene & 0.8 & 1.5 & 1.1 & 1.1 & 2.3 & 1.7 & 0.4 & 4.2 & 1.0 & 50,000 \\
\hline Tetrachloroethylene & 0.07 & 0.7 & 0.3 & ND & ND & ND & ND & 0.05 & 0.01 & 1,000 \\
\hline p- \& m-Xylene & 0.2 & 0.4 & 0.3 & 0.2 & 0.7 & 0.5 & ND & 0.2 & 0.1 & 100,000 \\
\hline o-Xylene & ND & 0.2 & 0.1 & 0.1 & 0.2 & 0.2 & ND & ND & ND & 100,000 \\
\hline
\end{tabular}

ND - Not Detected

Note: All VOC concentrations in units of ppbV.

Note: For the purpose of this table, not detects are treated as zeros when calculating mean concentration.

${ }^{1}$ VOC TWA's in units of $\mathrm{ppb}$, tritium DAC in units of $\mu \mathrm{Ci} / \mathrm{ml}$. Normal convention calls for TWA's to be reported in units of ppm. These values have been converted to $\mathrm{ppb}$ to allow easy comparision to field data.

${ }^{2}$ Tritium Concentrations in units of $\mathrm{pCi} / \mathrm{m}^{3}\left(\mathrm{pCi} / \mathrm{m}^{3} / 10^{12}=\mu \mathrm{Ci} / \mathrm{ml}\right)$

${ }^{3}$ Simple asphixiant, no TWA established.

${ }^{4}$ No TWA established. 
TA-54 Dome Study

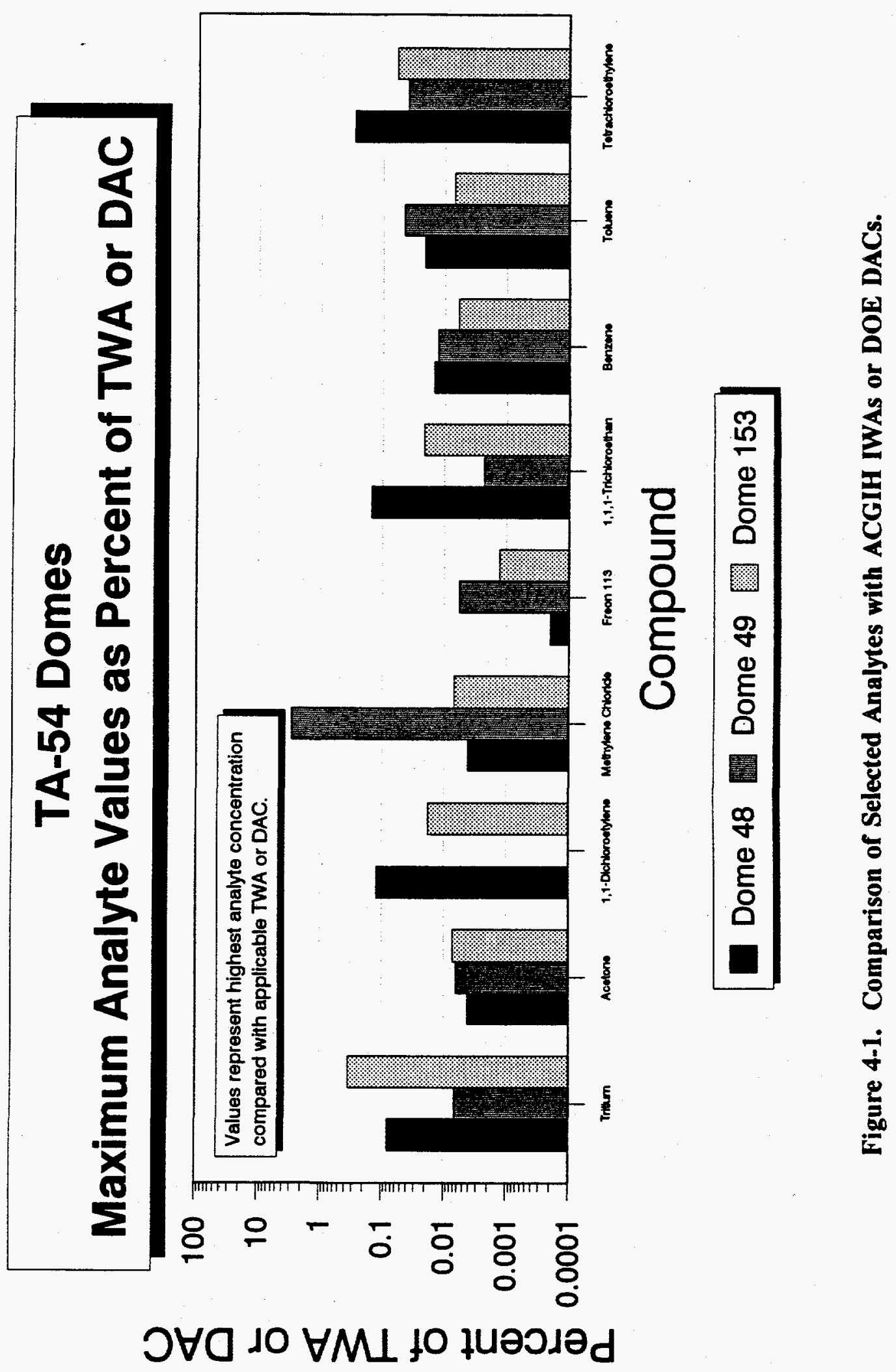




\subsection{Estimated Dome Emissions}

This study primarily evaluated the air concentrations within the storage domes. The data also can be used to estimate an emission rate if certain assumptions are made. Such an exercise has limitations. For instance we don't know the degree, if any, of the vertical compound stratification, what the source term is (i.e., what are the constant emission rates), and to what degree is air being exchanged within the dome.

The approach used to estimate emission rates includes the following assumptions:

- The integrated concentrations of tritium and VOCs measured represent steady state concentrations (i.e., 8-hour integrated averages are equal to 24hour integrated values);

- When the domes are closed, there are zero emissions;

- $\quad$ The domes are opened 7 days/week and emit $100 \%$ of the measured concentrations;

- The tritium and VOC concentrations are uniformly distributed both horizontally (which is generally indicated by the data) and vertically; and

- $\quad$ The dome ventilation, or air exchange rate, is one volume per day.
- The differences between analyte concentrations after 16 hour closure and 48 hour closure are not significant.

Some of these assumptions are realistic, while insufficient data do not allow the assessment of the other assumptions. The following equation was used to estimate annual emissions:

$$
\mathrm{E}=\mathrm{C}_{1} * \mathrm{~V} * 365 * \sigma / 1,000,000
$$

where:

$$
\begin{aligned}
& \mathrm{E}=\quad \begin{array}{l}
\text { Emission rate }(\mathrm{g} / \text { year for } \\
\text { VOCs and } \mu \mathrm{Ci} / \text { year for } \\
\text { tritium); }
\end{array} \\
& \mathrm{C}_{1}=\quad \begin{array}{l}
\text { 24-hour (average) analyte } \\
\text { concentration when dome } \\
\text { closed }\left(\mu \mathrm{g} / \mathrm{m}^{3}\right. \text { for VOCs, } \\
\mathrm{pCi} / \mathrm{m}^{3} \text { for tritium); }
\end{array} \\
& \sigma=\quad \begin{array}{l}
\text { Ventilation rate, assumed to } \\
\text { be once per day. }
\end{array} \\
& \mathrm{V}=\quad \begin{array}{l}
\text { Volume of dome }\left(\mathrm{m}^{3}\right) ; \\
365=
\end{array} \quad \begin{array}{l}
\text { Number of days in a year; } \\
\text { and }
\end{array} \\
& 1,000,000=\begin{array}{l}
\text { Conversion factor }(\mu \mathrm{g} / \mathrm{g} \text { or } \\
\mathrm{pCi} / \mu \mathrm{Ci}) .
\end{array}
\end{aligned}
$$

Table 4-6 shows estimated dome emissions for tritium and selected VOCs. The total estimated tritium emissions are $0.3 \mathrm{Ci} /$ year, which is only a small fraction of what is being released from the soils annually. The two major VOC emitters were methylene chloride and 1,1,1trichloroethane. The estimated annual 
Table 4-6

Summary of Annual Dome Emissions for Tritium and Selected VOCs

\begin{tabular}{|c|c|c|c|c|c|c|c|c|c|}
\hline \multirow{2}{*}{ Pone } & \multirow{2}{*}{$\begin{array}{l}\text { Est } \\
\text { (nolino }\end{array}$} & \multirow{2}{*}{  } & \multicolumn{7}{|c|}{ VOC Enissions (crams/year) } \\
\hline & & & Propans & Brominnertine & $\begin{array}{l}\text { Metiygene } \\
\text { Chloride }\end{array}$ & $17+0111$ & Trichloroothane & Benzene & Toluene \\
\hline Dome 48 & 8,588 & 0.0364 & 27.7 & 0.0 & 19.6 & 50.4 & 7,360 & 8.0 & 78.0 \\
\hline Dome 49 & 12,972 & 0.00285 & 5,060 & 0.0 & 13,000 & 1,100 & 173 & 15.1 & 353 \\
\hline Dome 153 & 13,527 & 0.2850 & 25.8 & 771 & 48.0 & 352 & 1,180 & 7.9 & 50.2 \\
\hline Total & & 0.324 & 5,110 & 771 & 13,100 & 1,500 & 8,710 & 31.0 & 481 \\
\hline
\end{tabular}


methylene chloride emissions from the three domes were 13,000 grams or approximately 30 pounds. The $1,1,1-$ trichloroethane estimated annual emissions were 8,700 grams or approximately 20 pounds. Remaining VOCs are estimated to each be emitted at a rate of several pounds per year, or less. Based on these data, the storage domes within TA-54 are relatively insignificant emission sources.

These estimates do not account for any pollutant losses through the dome roofs or walls, so more conservative annual emission estimates would be $2-3 x$ the calculated values. 


\subsection{CONCLUSIONS AND RECOMMENDATIONS}

The data generated during this study indicate that current operational procedures are adequate to protect workers from concentrations of analytes that exceed acceptable exposure limits. Standard operating procedures call for the domes to be open when work is being performed to reduce concentrations of constituents inside the domes. The data indicate that concentrations of tritium and VOCs increase considerably when the domes are closed. The values measured when the domes were closed, however, are still well below applicable ACGIH standards and when the domes were open, the values were up to two orders of magnitude lower.

The major VOC constituents were 1,1,1-trichloroethane (TCA) and methylene chloride. In the mixed waste storage dome (Dome 49) methylene chloride concentrations of up to $1.5 \mathrm{ppm}$ were recorded. Even this value was less than $3 \%$ of the ACGIH TWA. When the dome was open, the values were an order of magnitude $(0.13 \mathrm{ppm})$ less. The highest TCA values were measured in Dome 48. These values were almost three orders of magnitude lower than the TWA, e.g., highest measured concentration was 0.53 ppm and the TWA is $350 \mathrm{ppm}$.

The highest tritium value recorded, approximately $70,000 \mathrm{pCi} / \mathrm{m}^{3}$, was measured in Dome 153. The DAC for tritium is $20,000,000 \mathrm{pCi} / \mathrm{m}^{3}$, so this value is only $0.4 \%$ of the DAC. Even considering other exposures at LANL, this value does not pose a significant heath risk to workers or account for a significant portion of allowable annual exposure.

Emission estimates from the three domes show that the domes are relatively insignificant emitters of VOCs and tritium. Methylene chloride and TCA account for approximately 50 pounds per year of VOCs with all remaining VOCs contributing less than that amount. The total VOC emissions from all three domes are easily less than 100 pounds per year. The total tritium emissions for all three domes is approximately 0.3 Curies per year. The estimated tritium emissions for Area $G$, based on tritium flux monitoring, was 14 Curies per year. Therefore, the storage domes represent a relatively small point source.

While all data collected so far indicate the domes pose no worker hazard and are relatively small emitters of VOCs and tritium, several questions still remain unanswered. The reasons that tritium and VOC concentrations do not increase the longer they remain closed tends to indicate there is more air flow inside the domes than thought. Either the domes are well mixed or there is air flow into and out of the domes when they are closed preventing concentrations to build up higher than observed. Additionally, the vertical stratification, if any, is not known.

Emission estimates could be further improved by collecting 24-hour as well as 8-hour samples. Additionally, periodic grab samples could be used to try and assess the rate at which VOC compounds are building up and when steady state is actually reached. This should be done on a single dome only (i.e., Dome 49). The 
assessment of vertical stratification could also be performed.

This same approach would also be good to do for the tritium, however, it isn't certain that this approach is technically feasible due to sample collection constraints. The VOC data, however, could be used to extrapolate the tritium trends. 
APPENDIX A

LANL Sampling Master Log 

LANL Dome Sampling Master Log

\begin{tabular}{|c|c|c|c|c|c|c|c|c|c|c|}
\hline Date & $\begin{array}{l}\text { Sample } \\
\text { ID }\end{array}$ & $\begin{array}{c}\text { Sample } \\
\text { Type }\end{array}$ & $\begin{array}{l}\text { Grid } \\
\text { Point }\end{array}$ & $\begin{array}{l}\text { Canister } \\
\text { Number }\end{array}$ & $\begin{array}{l}\text { Silica Gel } \\
\text { Tube \#s }\end{array}$ & $\begin{array}{c}\text { Sampling } \\
\text { Time }\end{array}$ & $\begin{array}{l}\text { Elasped } \\
\text { Time } \\
\text { (hh:mu) }\end{array}$ & $\begin{array}{l}\text { Weight } \\
\text { Gain } \\
\text { (g) }\end{array}$ & $\begin{array}{l}\text { Sample } \\
\text { Volume } \\
\text { (fi3) }\end{array}$ & Comments \\
\hline $06 / 10 / 94$ & A347 & Silica Gel & $48-A$ & - & $002 / 065$ & 0803-1534 & $7: 31$ & 6.12 & 55.52 & \\
\hline $06 / 10 / 94$ & A348 & Silica Gel & $48-B$ & -- & $024 / 014$ & $0807-1538$ & $7: 31$ & 6.31 & 55.31 & \\
\hline $06 / 10 / 94$ & A349 & Silica Gel & $48-B$ & - & $011 / 030$ & $0807-1537$ & $7: 30$ & 6.79 & 58.71 & Duplicate \\
\hline $06 / 10 / 94$ & A350 & Silica Gel & $153-A$ & -- & $068 / 044$ & $0744-1518$ & $7: 34$ & 5.17 & 49.36 & \\
\hline $06 / 10 / 94$ & A351 & Silica Gel & 153-B & - & $025 / 035$ & $0748-1521$ & $7: 33$ & 5.51 & 56.67 & \\
\hline $06 / 10 / 94$ & A352 & Silica Gel & 153-C & - & $072 / 061$ & $0748-1524$ & $7: 35$ & 5.55 & 51.92 & \\
\hline $06 / 10 / 94$ & A353 & Canister & $48-\mathrm{A}$ & 1971 & -- & 0803-1534 & N/A & N/A & N/A & \\
\hline $06 / 10 / 94$ & A354 & Canister & $48-B$ & 1987 & -- & $0807-1538$ & N/A & N/A & N/A & \\
\hline $06 / 10 / 94$ & A355 & Canister & 48-B & 1990 & - & $0807-1537$ & N/A & N/A & N/A & Duplicate \\
\hline $06 / 10 / 94$ & A356 & Canister & 153-A & 1967 & -- & $0744-1518$ & N/A & N/A & N/A & \\
\hline $06 / 10 / 94$ & A357 & Canister & 153-B & 1988 & - & $0748-1521$ & N/A & N/A & N/A & \\
\hline $06 / 10 / 94$ & A358 & Canister & $153-C$ & 2000 & - & $0749-1524$ & N/A & N/A & N/A & \\
\hline $06 / 13 / 94$ & A360 & Silica Gel & $48-A$ & - & $025 / 027$ & $0800-1613$ & $8: 13$ & 7.46 & 63.36 & \\
\hline $06 / 13 / 94$ & A361 & Silica Gel & 48-B & - & $009 / 015$ & $0804-1616$ & $8: 12$ & 7.83 & 61.87 & \\
\hline $06 / 13 / 94$ & A362 & Silica Gel & 48-B & - & $041 / 036$ & 0803-1615 & $8: 12$ & 7.76 & 64.80 & Duplicate \\
\hline $06 / 13 / 94$ & A363 & Silica Gel & 153-A & - & $056 / 032$ & $0734-1550$ & $8: 16$ & 6.65 & 60.75 & \\
\hline $06 / 13 / 94$ & A364 & Silica Gel & 153-B & - & $039 / 057$ & $0738-1554$ & $8: 16$ & 6.38 & 60.47 & \\
\hline $06 / 13 / 94$ & A365 & Silica Gel & $153-C$ & - & $060 / 026$ & $0743-1600$ & $8: 17$ & 6.54 & 60.08 & \\
\hline $06 / 13 / 94$ & A366 & Canister & $48-\mathrm{A}$ & 2005 & -- & $0757-1613$ & N/A & N/A & N/A & \\
\hline $06 / 13 / 94$ & A367 & Canister & 48-B & 1975 & - & $0800-1616$ & N/A & N/A & N/A & \\
\hline $06 / 13 / 94$ & A368 & Canister & 48-B & 1996 & - & $0803-1615$ & N/A & N/A & N/A & Duplicate \\
\hline $06 / 13 / 94$ & A369 & Canister & $153-A$ & 1993 & - & $0734-1550$ & N/A & N/A & N/A & \\
\hline $06 / 13 / 94$ & $\mathrm{~A} 370$ & Canister & 153-B & 2006 & -- & $0738-1554$ & N/A & N/A & N/A & \\
\hline
\end{tabular}




\section{LANL Master Log, Continued}

\begin{tabular}{|c|c|c|c|c|c|c|c|c|c|c|}
\hline Date & $\begin{array}{l}\text { Sample } \\
\text { ID }\end{array}$ & $\begin{array}{l}\text { Sample } \\
\text { Type }\end{array}$ & $\begin{array}{l}\text { Grid } \\
\text { Point }\end{array}$ & $\begin{array}{l}\text { Canister } \\
\text { Number }\end{array}$ & $\begin{array}{l}\text { Silica Gel } \\
\text { Tube \#s }\end{array}$ & $\begin{array}{c}\text { Sampling } \\
\text { Time }\end{array}$ & $\begin{array}{l}\text { Elasped } \\
\text { Time } \\
\text { (hh:mim) }\end{array}$ & $\begin{array}{l}\text { Weight } \\
\text { Gain } \\
\text { (g) }\end{array}$ & $\begin{array}{l}\text { Sample } \\
\text { Volume } \\
\text { (ft3) }\end{array}$ & Comments \\
\hline $06 / 13 / 94$ & $\mathrm{~A} 371$ & Canister & $153-\mathrm{C}$ & 1992 & - & 0743-1601 & N/A & N/A & N/A & \\
\hline \multirow[t]{2}{*}{$06 / 14 / 94$} & A379 & Silica Gel & $48-A$ & -- & $050 / 001$ & $0747-1629$ & 19:03 & 3.25 & 143.86 & \\
\hline & A380 & Silica Gel & 48-B & - & $055 / 042$ & $0750-1630$ & 19:06 & 3.81 & 143.16 & \\
\hline \multirow[t]{2}{*}{ TO } & A381 & Silica Gel & 153-A & -- & $028 / 024$ & $0730-1645$ & $19: 41$ & 3.01 & 139.10 & \\
\hline & A382 & Silica Gel & 153-B & -- & $072 / 035$ & 0738-1649 & $19: 37$ & 2.87 & 140.59 & \\
\hline $06 / 15 / 94$ & A383 & Silica Gel & 153-C & -- & $019 / 010$ & 0741-1655 & $19: 40$ & 3.12 & 130.44 & \\
\hline $06 / 14 / 94$ & A384 & Canister & $48-A$ & 2003 & -- & $0747-1614$ & $8: 27$ & N/A & N/A & \\
\hline $06 / 14 / 94$ & A385 & Canister & 48-B & 1962 & -- & $0750-1618$ & $8: 28$ & $N / A$ & N/A & \\
\hline $06 / 14 / 94$ & A386 & Canister & $153-A$ & 1970 & -- & 0730-1557 & $8: 27$ & N/A & N/A & \\
\hline $06 / 14 / 94$ & A387 & Canister & 153-B & 1973 & -- & 0735-1602 & $8: 27$ & N/A & N/A & \\
\hline $06 / 14 / 94$ & A388 & Canister & $153-C$ & 1966 & - & $0738-1605$ & $8: 27$ & N/A & N/A & \\
\hline $06 / 16 / 94$ & A404 & Canister & IN L & 1960 & - & $0830-1608$ & $7: 38$ & N/A & N/A & Bet. Rows 39 and 41 \\
\hline $06 / 16 / 94$ & $\mathrm{~A} 405$ & Canister & IN L & 1972 & - & $0827-1606$ & $7: 39$ & N/A & N/A & Bet. Rows 63 and 65 \\
\hline $06 / 16 / 94$ & A406 & Canister & IN L & 2002 & - & $0832-1610$ & $7: 38$ & N/A & N/A & End of Pad \\
\hline $07 / 28 / 94$ & A464 & Canister & 49-A & 2000 & -- & $0812-1652$ & $8: 40$ & N/A & N/A & Open Dome \\
\hline $07 / 28 / 94$ & A465 & Canister & 49-B & 1968 & - & 0816-1656 & $8: 40$ & N/A & N/A & Open Dome \\
\hline $07 / 28 / 94$ & A466 & Canister & 49-B & 1980 & - & $0816-1656$ & $8: 40$ & N/A & N/A & Open Dome, Duplicate \\
\hline $07 / 28 / 94$ & A467 & Canister & 48-A & 1967 & -- & $0740-1616$ & $8: 36$ & N/A & N/A & Open Dome \\
\hline $07 / 28 / 94$ & A468 & Canister & 48-B & 1964 & - & $0747-1619$ & $8: 32$ & N/A & N/A & Open Dome \\
\hline $07 / 28 / 94$ & A469 & Canister & $153-A$ & 1989 & - & $0757-1633$ & $8: 36$ & N/A & N/A & Open Dome \\
\hline $07 / 28 / 94$ & A470 & Canister & 153-B & 1992 & - & $0801-1637$ & $8: 36$ & N/A & N/A & Open Dome \\
\hline
\end{tabular}


LANL Master Log, Continued

\begin{tabular}{|c|c|c|c|c|c|c|c|c|c|c|}
\hline Date & $\begin{array}{l}\text { Sample } \\
\text { ID }\end{array}$ & $\begin{array}{l}\text { Sample } \\
\text { Type }\end{array}$ & $\begin{array}{l}\text { Grid } \\
\text { Point }\end{array}$ & $\begin{array}{l}\text { Canister } \\
\text { Number }\end{array}$ & $\begin{array}{c}\text { Silica Gel } \\
\text { Tube \#s }\end{array}$ & $\begin{array}{c}\text { Sampling } \\
\text { Time }\end{array}$ & $\begin{array}{l}\text { Elasped } \\
\text { Time } \\
\text { (hh:mm) }\end{array}$ & $\begin{array}{l}\text { Weight } \\
\text { Gain } \\
\text { (g) }\end{array}$ & $\begin{array}{l}\text { Sample } \\
\text { Volume } \\
\text { (ft3) }\end{array}$ & Comments \\
\hline $07 / 28 / 94$ & A471 & Canister & $153-C$ & 2006 & -- & $0805-1641$ & $8: 36$ & N/A & N/A & Open Dome \\
\hline $07 / 28 / 94$ & A477 & Silica Gel & 48-A & -- & $038 / 056$ & 0741-1417 & $6: 36$ & 10.11 & 43.89 & \\
\hline $07 / 28 / 94$ & A478 & Silica Gel & 48-B & -- & $055 / 005$ & $0745-1423$ & $6: 38$ & 9.75 & 43.61 & \\
\hline $07 / 28 / 94$ & A479 & Silica Gel & 48-B & - & $061 / 034$ & $0747-1427$ & $6: 40$ & N/A & 26.52 & Duplicate \\
\hline $07 / 28 / 94$ & A480 & Silica Gel & $49-A$ & - & $003 / 044$ & $0814-1518$ & $7: 04$ & 9.98 & 45.25 & \\
\hline $07 / 28 / 94$ & A481 & Silica Gel & 49-B & - & $041 / 050$ & $0846-1522$ & $6: 36$ & 10.22 & 47.29 & \\
\hline $07 / 28 / 94$ & A482 & Silica Gel & 49-C & - & $072 / 025$ & $0821-1526$ & $7: 05$ & 10.93 & 51.90 & \\
\hline $07 / 28 / 94$ & A483 & Silica Gel & 153-A & - & $037 / 036$ & 0759-1442 & $6: 19$ & 10.29 & 42.16 & \\
\hline $07 / 28 / 94$ & A484 & Silica Gel & 153-B & -- & $027 / 021$ & $0802-1445$ & $6: 43$ & 9.79 & 43.38 & \\
\hline $07 / 28 / 94$ & A485 & Silica Gel & $153-\mathrm{C}$ & 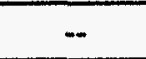 & $018 / 070$ & $0806-1447$ & $6: 41$ & 9.74 & 42.39 & \\
\hline $07 / 29 / 94$ & A489 & Silica Gel & 48-A & - & $031 / 002$ & 0740-1454 & $7: 14$ & 9.52 & 40.50 & Closed Dome \\
\hline $07 / 29 / 94$ & A490 & Silica Gel & 48-B & - & $026 / 024$ & $0745-1500$ & $7: 15$ & 9.40 & 38.53 & Closed Dome \\
\hline $07 / 29 / 94$ & A491 & Silica Gel & 48-B & - & $006 / 047$ & $0740-1500$ & $7: 20$ & 8.67 & 32.20 & Duplicate \\
\hline $07 / 29 / 94$ & A492 & Silica Gel & 49-A & - & $015 / 004$ & $0742-1515$ & $7: 33$ & 9.49 & 41.19 & Closed Dome \\
\hline $07 / 29 / 94$ & A493 & Silica Gel & 49-B & - & $058 / 076$ & 0749-1517 & $7: 28$ & 10.66 & 46.68 & Closed Dome \\
\hline $07 / 29 / 94$ & A494. & Silica Gel & $49-\mathrm{C}$ & -- & $074 / 001$ & $0754-1520$ & $7: 26$ & 10.68 & 49.38 & Closed Dome \\
\hline $07 / 29 / 94$ & A495 & Silica Gel & 153-A & - & $007 / 028$ & 0753-1315 & $5: 22$ & 8.74 & 24.93 & Closed Dome \\
\hline $07 / 29 / 94$ & A496 & Silica Gel & 153-B & - & $053 / 010$ & $0751-1515$ & $7: 24$ & 8.95 & 34.95 & Closed Dome \\
\hline $07 / 29 / 94$ & A497 & Silica Gel & $153-\mathrm{C}$ & - & $017 / 035$ & 0759-1517 & $7: 17$ & 8.50 & 33.01 & Closed Dome \\
\hline $07 / 29 / 94$ & A498 & Canister & 48-A & 1997 & - & $0738-1544$ & N/A & N/A & N/A & Closed Dome \\
\hline $07 / 29 / 94$ & A499 & Canister & 48-B & 2005 & -- & $0740-1543$ & N/A & N/A & N/A & Closed Dome \\
\hline $07 / 29 / 94$ & A500 & Canister & 49-A & 1998 & - & $0742-1600$ & N/A & N/A & N/A & Closed Dome \\
\hline $07 / 29 / 94$ & A501 & Canister & 49-B & 1995 & - & $0747-1602$ & N/A & N/A & N/A & Closed Dome \\
\hline
\end{tabular}




\section{LANL Master Log, Continued}

\begin{tabular}{|c|c|c|c|c|c|c|c|c|c|c|}
\hline Date & $\begin{array}{l}\text { Sample } \\
\text { ID }\end{array}$ & $\begin{array}{l}\text { Sample } \\
\text { Type }\end{array}$ & $\begin{array}{l}\text { Grid } \\
\text { Point }\end{array}$ & $\begin{array}{l}\text { Canister } \\
\text { Number }\end{array}$ & $\begin{array}{l}\text { Silica Gel } \\
\text { Tube \#s }\end{array}$ & $\begin{array}{c}\text { Sampling } \\
\text { Time }\end{array}$ & $\begin{array}{l}\text { Elasped } \\
\text { Time } \\
\text { (hh:mm) }\end{array}$ & $\begin{array}{l}\text { Weight } \\
\text { Gain } \\
\text { (g) }\end{array}$ & $\begin{array}{c}\text { Sample } \\
\text { Volume } \\
\text { (fi3) }\end{array}$ & Comments \\
\hline $07 / 29 / 94$ & A502 & Canister & 49-B & 1974 & - & 0747-1602 & N/A & N/A & N/A & Duplicate \\
\hline $07 / 29 / 94$ & A503 & Canister & 49-C & 1999 & - & 0752-1559 & N/A & N/A & N/A & Closed Dome \\
\hline $07 / 29 / 94$ & A504 & Canister & 153-A & 1979 & - & $0753-1352$ & N/A & N/A & N/A & Closed Dome \\
\hline $07 / 29 / 94$ & A505 & Canister & 153-B & 1990 & -- & $0751-1350$ & N/A & N/A & N/A & Closed Dome \\
\hline $07 / 29 / 94$ & A506 & Canister & $153-\mathrm{C}$ & 1986 & - & $0759-1551$ & N/A & N/A & N/A & Closed Dome \\
\hline $08 / 01 / 94$ & A513 & Canister & $48-\mathrm{A}$ & 1970 & $\therefore$ & 0734-1531 & $7: 57$ & N/A & N/A & \\
\hline $08 / 01 / 94$ & A514 & Canister & 48-B & 1962 & - & 0735-1534 & $7: 59$ & N/A & N/A & \\
\hline $08 / 01 / 94$ & A515 & Canister & 49-A & 1984 & - & $0738-1600$ & $8: 22$ & N/A & N/A & Closed Dome \\
\hline $08 / 01 / 94$ & $\mathrm{~A} 516$ & Canister & 49-B & 1976 & - & $0743-1602$ & $8: 19$ & N/A & N/A & Closed Dome \\
\hline $08 / 01 / 94$ & A517 & Canister & 49-B & 1987 & - & 0743-1602 & $8: 19$ & N/A & N/A & Closed Dome, Dupliate \\
\hline $08 / 01 / 94$ & A518 & Canister & 49-C & 2004 & - & $0748-1606$ & $8: 18$ & N/A & N/A & Closed Dome \\
\hline $08 / 01 / 94$ & A519 & Canister & 153-A & 1959 & - & 0749-1542 & $7: 53$ & N/A & N/A & \\
\hline $08 / 01 / 94$ & A520 & Canister & 153-B & 1983 & -- & $0750-1544$ & $7: 26$ & N/A & N/A & \\
\hline $08 / 01 / 94$ & A521 & Canister & $153-\mathrm{C}$ & 1975 & - & 0753-1546 & $7: 53$ & N/A & N/A & \\
\hline $08 / 01 / 94$ & A522 & Silica Gel & $48-A$ & - & $018 / 066$ & 0734-1506 & $7: 32$ & 10.55 & 39.70 & Closed Dome \\
\hline $08 / 01 / 94$ & A523 & Silica Gel & 48-B & - & $034 / 064$ & $0736-1534$ & $7: 58$ & 9.95 & 37.40 & Closed Dome \\
\hline $08 / 01 / 94$ & A524 & Silica Gel & 48-B & -- & $073 / 051$ & $0735-1510$ & 7:35 & 10.61 & 34.74 & Closed Dome, Duplicate \\
\hline $08 / 01 / 94$ & A525 & Silica Gel & 49-A & - & $040 / 003$ & $0738-1600$ & $8: 22$ & 9.34 & 34.25 & Closed Dome \\
\hline $08 / 01 / 94$ & A526 & Silica Gel & 49-B & - & $065 / 063$ & 0743-1602 & $8: 19$ & 10.52 & 17.52 & Closed Dome \\
\hline $08 / 01 / 94$ & A527 & Silica Gel & 49-C & -- & $021 / 033$ & $0748-1606$ & $8: 18$ & 11.63 & 44.57 & Closed Dome \\
\hline $08 / 01 / 94$ & A528 & Silica Gel & $153-A$ & - & $032 / 050$ & 0749-1542 & $7: 53$ & 9.92 & 35.49 & Closed Dome \\
\hline $08 / 01 / 94$ & A529 & Silica Gel & 153-B & - & $005 / 062$ & $0750-1544$ & $7: 54$ & 10.75 & 47.47 & Closed Dome \\
\hline $08 / 01 / 94$ & A530 & Silica Gel & 153-C & - & $025 / 042$ & $0753-1546$ & $7: 53$ & 9.22 & 33.42 & Closed Dome \\
\hline
\end{tabular}




\section{DISCLAIMER}

This report was prepared as an account of work sponsored by an agency of the United States Government. Neither the United States Government nor any agency thereof, nor any of their employees, makes any warranty, express or implied, or assumes any legal liability or responsibility for the accuracy, compieteness, or usefulness of any information, apparatus, product, or process disclosed, or represents that its use would not infringe privately owned rights. Reference herein to any specific commercial product, process, or service by trade name, trademark, manufacturer, or otherwise does not necessarily constitute or imply its endorsemeat, recommendation, or favoring by the United States Government or any agency thereof. The views and opinions of authors expressed herein do not necessarily state or reflect those of the United States Government or any agency thereof. 